\title{
Amenable groups without finitely presented amenable covers
}

\author{
Mustafa Gökhan Benli • Rostislav Grigorchuk • \\ Pierre de la Harpe
}

Received: 10 June 2012 / Revised: 25 June 2012 / Accepted: 2 February 2013 /

Published online: 1 May 2013

(C) The Author(s) 2013. This article is published with open access at SpringerLink.com

\begin{abstract}
The goal of this article is to study results and examples concerning finitely presented covers of finitely generated amenable groups. We collect examples of groups $G$ with the following properties: (i) $G$ is finitely generated, (ii) $G$ is amenable, e.g. of intermediate growth, (iii) any finitely presented group with a quotient isomorphic to $G$ contains non-abelian free subgroups, or the stronger (iii') any finitely presented group with a quotient isomorphic to $G$ is large.
\end{abstract}

Keywords Finitely generated groups - Finitely presented covers - Self-similar groups - Metabelian groups - Soluble groups - Groups of intermediate growth · Amenable groups · Groups with non-abelian free subgroups · Large groups

Mathematics Subject Classification (2000) $\quad$ 20E08 · 20E22 · 20F05 · 43A07

Communicated by Dr. Efim Zelmanov.

We are grateful to the Swiss National Science Foundation for supporting a visit of the first author to Geneva. The first author is grateful to the department of mathematics, University of Geneva, for their hospitality during his stay. The first and the second authors are partially supported by NSF grant DMS 1207699. The second author was supported by ERC starting grant GA 257110 "RaWG".

M. G. Benli $(\bowtie) \cdot$ R. Grigorchuk

Department of Mathematics, Texas A\&M University, Mailstop 3368,

College Station, TX 77843-3368, USA

e-mail: mbenli@math.tamu.edu

R. Grigorchuk

e-mail: grigorch@math.tamu.edu

P. de la Harpe

Section de mathématiques, Université de Genève, C.P. 64, 1211 Genève 4, Switzerland

e-mail: Pierre.delaHarpe@unige.ch 


\section{Introduction}

1.A. Motivation In the study of finiteness conditions on groups, the following type of question is natural:

Question 1.1 Given a Property $(\mathcal{P})$ of groups, does any finitely generated group with $(\mathcal{P})$ have a finitely presented cover with $(\mathcal{P})$ ?

A cover of a group $G$ is a group $E$ given together with an epimorphism $E \rightarrow G$. A cover fits into an extension $1 \rightarrow N \rightarrow E \rightarrow G \rightarrow 1$.

The answer can be positive for trivial reasons, for example when Property $(\mathcal{P})$ holds for free groups (such as exponential growth) or when Property $(\mathcal{P})$ implies finite presentation (such as nilpotency, or polynomial growth). The answer is also positive when $(\mathcal{P})$ is Kazhdan's Property $(\mathcal{T})$, by a non-trivial result of Shalom [137].

Here, we concentrate on Question 1.1 for amenability, a case in which the answer is negative. Indeed, the goal of this article is to study examples and results concerning finitely generated amenable groups that do not have finitely presented amenable covers.

Our motivation is better to understand the class of finitely presented amenable groups, and related groups. To describe the situation, it is convenient to introduce the class $\mathcal{A G}$ of amenable groups, and the subclass $\mathcal{E} \mathcal{G}$ of elementary amenable groups, which is easier to understand (Appendix D is a reminder on these classes, and on growth).

On the one hand, many examples are known in $\mathcal{E} \mathcal{G}$ of finitely presented soluble groups, including the standard examples described in the exposition [145], the Baumslag-Remeslennikov group, which is a finitely presented metabelian group with derived group free abelian of infinite rank (references in our Remark A.4.c), and the Kharlampovich group, which is a finitely presented soluble group with unsoluble word problem (references in the proof of Proposition A.5). There are also finitely presented groups in $\mathcal{E} \mathcal{G}$ which are not virtually soluble (Example D.1).

In the complement $\mathcal{A G} \backslash \mathcal{E} \mathcal{G}$, a reasonable number of finitely generated examples are known, in particular the groups of intermediate growth. Also known are finitely presented examples, all closely related to self-similar groups. But not enough examples are known to allow one to guess at a general picture; this is illustrated by the following old and basic problem in the theory of growth of groups (see, e.g., [105], Problems 4.5.b, 9.8, and 10.11):

Basic problem 1.2 Does there exist a finitely presented group of intermediate growth?

The answer does not seem to be at hand. Here is a possibly easier problem:

Problem 1.3 Does there exist a finitely generated group of intermediate growth that is the quotient of a finitely presented group without non-abelian free subgroups? or the quotient of a finitely presented amenable group?

Finitely generated elementary amenable groups are never of intermediate growth [48], so that Problems 1.2 and 1.3 do not arise in $\mathcal{E} \mathcal{G}$. Problem 1.3 involves two questions, because there exist finitely presented groups that are non-amenable and do not contain non-abelian free subgroups [126]. 
Strictly speaking, the solution of the analogue of Problem 1.2 is known for the class $\mathcal{A G} \backslash \mathcal{E} \mathcal{G}$. Indeed, one knows a few sporadic examples of finitely presented groups in this class, such as the HNN-extension $\widehat{\mathfrak{G}}$ of the Grigorchuk group [70], and the HNN-extension of the Brunner-Sidki-Vieira group that appears in [81, Proposition 6] (the latter group is amenable by [13] and not elementary amenable by [81]). The finitely presented HNN-extension of the basilica group that appears in [81, Theorem 1.7] is not even in the class $\mathcal{A G} \backslash \mathcal{S G}$, defined in Appendix D; see also [10, Theorem 12]. But, despite these examples, very little is known about finitely presented groups in $\mathcal{A G} \backslash \mathcal{E} \mathcal{G}$, and it would be interesting to find methods providing new examples; a priori, one could hope and try finitely presented covers, but our paper shows that this does not look very promising.

Before stating the next problem, here is a definition: the elementary amenable radical $\operatorname{Rad}_{\mathrm{ea}}(G)$ of a group $G$ is its unique maximal normal elementary amenable subgroup. Note that $\operatorname{Rad}_{\mathrm{ea}}(G)$ is contained in the amenable radical of $G$, that appears (but for its name) in [56, Lemma 1 of Section 4].

Problem 1.4 Is there a finitely presented group $G$ in $\mathcal{A G} \backslash \mathcal{E} \mathcal{G}$, with $\operatorname{Rad}_{\mathrm{ea}}(G)=\{1\}$, that has infinitely presented ${ }^{1}$ quotients? or that has uncountably many pairwise nonisomorphic quotients?

1.B. First examples When $(\mathcal{P})$ is amenability, we know two approaches to Question 1.1: One is based on the theory of $\Sigma$-invariants, developed in a series of papers by Bieri, Strebel, and Neumann (see Appendix C). The other involves self-similar groups and approximation methods (see Sections 2, 3).

The first result we quote is due to Bieri and Strebel [31, Theorem 5.5 and Corollary 5.6]. On the one hand, we reformulate it in a slightly weaker version, assuming that $E$ is finitely presented (instead of assuming that $E$ has Property $\mathrm{FP}_{2}$, as in the original paper); on the other hand, we formulate it for virtually metabelian groups, because this follows immediately from the case of metabelian groups.

Theorem 1.5 (Bieri-Strebel) Let $G$ be a virtually metabelian group that is finitely generated and infinitely presented.

Any finitely presented cover $E$ of $G$ has non-abelian free subgroups. In particular, $E$ is non-soluble, indeed non-amenable.

The proof of Theorem 1.5 can now be understood in a much simpler way than in [31], as we indicate in Corollary C.4. The reason is that we can use better invariants, namely those defined in [32] and their later reformulations (see Strebel's exposition in progress [146]).

Examples of metabelian groups that are finitely generated and infinitely presented include matrix groups like $\left(\begin{array}{ll}\left.\left(\frac{\ell}{m}\right)^{\mathbf{Z}} \mathbf{Z}\left[\frac{1}{\ell m}\right]\right) \\ 0 & 1\end{array}\right)$ where $\ell, m \geq 2$ are coprime integers, wreath products $A<\mathbf{Z}$ where $A \neq\{1\}$ is a finitely generated abelian group, and free metabelian groups $\operatorname{FSol}(k, 2):=F_{k} /\left[\left[F_{k}, F_{k}\right],\left[F_{k}, F_{k}\right]\right]$, where $F_{k}$ is the free group on $k \geq 2$ generators.

\footnotetext{
1 A finitely generated group is "infinitely presented" if it is not finitely presented.
} 
Appendix A includes a reminder on metabelian groups. Appendix B is a reminder on wreath products and lamplighter groups. Appendix $\mathrm{C}$ is a reminder on BieriNeumann-Strebel invariants. Proposition B.2, Corollary B.3, Proposition B.9, Corollary C.4, and Corollary C.6 give examples of finitely generated groups of which all finitely presented covers have non-abelian free subgroups.

We denote by $\mathfrak{G}$ the Grigorchuk group, ${ }^{2}$ introduced in [66]; see Example 2.16. Recall here that $\mathfrak{G}$ is finitely generated, indeed generated by four involutions traditionally written $a, b, c, d$. This group has many remarkable properties, including that of being an infinite 2-group of intermediate growth; in particular it is amenable. The group $\mathfrak{G}$ has a presentation, due to Lysenok, with four generators and infinitely many relators. Appropriate finite subsets of these relators naturally define a sequence $\left(\mathfrak{G}_{n}\right)_{n \geq 0}$ of four-generated finitely presented groups converging to $\mathfrak{G}$ (Definition 5.2).

There is a reminder on convergence of groups in Section 3.

Theorem 1.6 [78] Any finitely presented cover of the group $\mathfrak{G}$ is large.

Recall that a group is large if it contains a subgroup of finite index that has nonabelian free quotients. Note that large groups have non-abelian free subgroups.

In view of Proposition 3.3 below, Theorem 1.6 is a straightforward consequence of the main result of [78]; see also Theorem 2.14 and Example 2.16. More precisely, it was shown in [78] that each $\mathfrak{G}_{n}$ is virtually a direct product of finitely generated non-abelian free groups, this has been sharpened in [11], and can be further improved: Theorem 1.7 (Section 5) Let $\mathfrak{G}$ and $\mathfrak{G}_{n}$ be as above. For each $n \geq 0$, the group $\mathfrak{G}_{n}$ has a normal subgroup $H_{n}$ of index $2^{2^{n+1}+2}$ that is isomorphic to the direct product of $2^{n}$ free groups of rank 3.

Another way of sharpening Theorem 1.6 is given in Proposition 5.11, where the condition of finite presentability for the cover of $\mathfrak{G}$ is replaced by the weaker Condition $\mathrm{FP}_{2}$.

We denote by $\mathfrak{B}$ the Basilica group. Recall here that $\mathfrak{B}$ can be generated by two elements, and is an amenable torsion-free group of exponential growth. See Example 2.17 and Appendix D for some other properties of $\mathfrak{B}$.

Theorem 1.8 (Erschler) Any finitely presented cover of the Basilica group $\mathfrak{B}$ has non-abelian free subgroups.

Given an invertible automaton $(A, \tau)$ over a finite alphabet $X$, Erschler introduces a notion of "automatically presented group $G^{*}(A, \tau)$ generated by the automaton" (see [60] for details). She shows that, if $G^{*}(A, \tau)$ is not virtually abelian, then any finitely presented cover of it has non-abelian free subgroups. For the Basilica automaton, the group $G^{*}(A, \tau)$ coincides with $\mathfrak{B}$, and Theorem 1.8 follows.

As noted in [60], these arguments do not apply to the group $\mathfrak{G}$ of Theorem 1.6; this is due to the fact that, for $(A, \tau)$ the automaton of $\mathfrak{G}$, the cover $G^{*}(A, \tau) \rightarrow \mathfrak{G}$ has a non-trivial kernel.

In our setting, for $G=\mathfrak{B}$, the universal contracting cover $G_{0}$ of Definition 2.5 is free of rank 2 (see Example 2.17), and Theorem 1.8 follows from our Theorem 2.14. Indeed, our argument shows that any finitely presented cover of $\mathfrak{B}$ is large.

\footnotetext{
2 Two of the authors insist that we mention this terminology, often used today. The third author submits.
} 
Notation: In this paper, the symbols $\mathfrak{G}$ and $\mathfrak{B}$ will be used only for the two groups introduced above. In Examples 2.18 to 2.21, we introduce four other groups, with their usual notation in this subject: the iterated monodromy group of $z^{2}+i$ denoted by $\mathfrak{J}$, the Gupta-Sidki group $\mathfrak{G} \mathfrak{S}$, the Fabrykowski-Gupta group $\mathfrak{F} \mathfrak{G}$, and the Hanoi Towers group $\mathfrak{H}$.

We show in Section 2 that Theorem 2.14 implies Theorems 1.6 on $\mathfrak{G}, 1.8$ on $\mathfrak{B}$, and 2.22 on $\mathfrak{J}, \mathfrak{G} \mathfrak{S}, \mathfrak{F} \mathfrak{G}$, and $\mathfrak{H}$. We state now a shorthand version of 2.14. Notation and technical terms are defined in Section 2.

Theorem 1.9 Let $G$ be an infinite finitely generated self-similar group. Assume that $G$ is contracting faithful self-replicating. Let $G_{0}$ denote a standard contracting cover of $G$, as in Definition 2.8. $G$.

If $G_{0}$ has non-abelian free subgroups, then so does any finitely presented cover of

If $G_{0}$ is large, any finitely presented cover of $G$ is large.

1.C. Infinitely more examples The group $\mathfrak{G}$ has uncountably many ${ }^{3}$ relatives $G_{\omega}$ introduced in [68]. Each of these groups is generated by a set $S_{\omega}$ of four involutions. They are parameterized by the space $\Omega=\{0,1,2\}^{\mathbf{N}}$. We have $\mathfrak{G}=G_{\omega}$ when $\omega$ is the 3 -periodic sequence $012012012 \cdots$. Let $\Omega_{+}$denote the complement in $\Omega$ of the space of eventually constant sequences. Section 4 contains a description of the family $\left(G_{\omega}\right)_{\omega \in \Omega_{+}}$.

Theorem 1.10 For $\omega \in \Omega_{+}$, the group $G_{\omega}$ is of intermediate growth, and any finitely presented cover of $G_{\omega}$ is large.

Note also the following straightforward consequence of Theorems 1.6, 1.8, and 1.10:

Corollary 1.11 Let $H$ be a finitely generated cover of one of $\mathfrak{G}$ (as in Theorem 1.6), $\mathfrak{B}$ (as in Theorem 1.8), or $G_{\omega}, \omega \in \Omega_{+}$(as in Theorem 1.10). Any finitely presented cover of $H$ is large.

There are several interesting classes of groups that qualify to be the $H$ of Corollary 1.11:

(i) The uncountably many groups of [59], which are finitely generated, of intermediate growth, and not residually finite, each one being a central cover of $\mathfrak{G}$.

(ii) The groups of [14], which are finitely generated groups of intermediate growth, with exactly known growth functions, each one being a cover of $\mathfrak{G}$.

(iii) Permutational wreath products of the form $A_{{ }_{2 X}} G_{\omega}$, where $A \neq\{1\}$ is a finite group and $G_{\omega}$ is as in Theorem 1.10 [15].

There is an uncountable family of finitely generated amenable simple groups, which are topological full groups of minimal homeomorphisms of the Cantor space [100]. None of these groups is finitely presented (see [114, Theorem 5.7], as well as [86]). In our context, it is natural to formulate:

\footnotetext{
${ }^{3}$ Here and elsewhere, "uncountably many" groups means "uncountably many pairwise non-isomorphic" groups.
} 
Problem 1.12 Let $G$ be one of the finitely generated amenable simple groups that appears in [100]. Does $G$ have an amenable finitely presented cover?

Remark 1.13 We state two kinds of results concerning appropriate covers: some establish that the covers are large (see Theorem 1.6), others, weaker, that they contain non-abelian free groups (see Theorem 1.5). Strong statements do not hold in all cases.

For example, let $G=\operatorname{Met}(k, \ell) \simeq \mathbf{Z}\left[\frac{1}{\ell m}\right] \rtimes_{\ell / m} \mathbf{Z}$, for two coprime integers $k$, $\ell \geq 2$ (Definition B.7). Then $G$ satisfies the hypothesis of Theorem 1.5. The Baumslag-Solitar group $\mathrm{BS}(\ell, m)$ is a finitely presented cover of $G$; it is known that $\mathrm{BS}(\ell, m)$ has non-abelian free subgroups, but is not large (Proposition B.6.iv).

1.D. A dual to Question 1.1 Given a Property $(\mathcal{P})$ of groups, it is standard to ask whether any countable group with $(\mathcal{P})$ is a subgroup of some finitely generated group with $(\mathcal{P})$. For example, the answer is known to be positive if $(\mathcal{P})$ is solubility [122], or amenability, or elementary amenability [128, Corollary 1.3]. An earlier result of [96] also gives a positive answer to a similar question: for a given integer $n$, if $G$ is a group that has a presentation with a countable number (possibly infinite) of generators and $n$ relators, then $G$ can be embedded in a finitely presented group with 2 generators and $n$ relators. But the answer is negative if $(\mathcal{P})$ is nilpotency, because any subgroup of a finitely generated nilpotent group is finitely generated. It is also negative if $(\mathcal{P})$ is metabelianity: indeed, for any prime $p$, the abelian quasi-cyclic group $\mathbf{Z}\left(p^{\infty}\right)$ cannot be embedded in a finitely generated metabelian group [122, Lemma 5.3].

As a digression from our main theme, and since recursively presented groups are mentioned in Appendix A, we formulate one more question, which is in some sense dual to Question 1.1.

Question 1.14 Given a Property $(\mathcal{P})$ of groups, is any finitely generated recursively presented group with $(\mathcal{P})$ a subgroup of some finitely presented group with $(\mathcal{P})$ ?

The answer to Question 1.14 is known to be positive if $(\mathcal{P})$ is metabelianity (Proposition A.2), or solubility of the word problem ([49], and also [117, Theorem 2.8]). The answer is not known if $(\mathcal{P})$ is amenability [128, Problem 1.7]. NP

1.E. Plan of the paper Section 2: Non-abelian free subgroups of finitely presented covers of contracting self-similar groups. Proofs of Theorems 1.9 and 2.14; proofs of Theorems 1.6, 1.8, and their analogues for $\mathfrak{J}, \mathfrak{G} \mathfrak{S}, \mathfrak{F} \mathfrak{G}$, and $\mathfrak{H}$.

Section 3: Marked groups and the Chabauty topology, a reminder.

Section 4: The analogue of Theorem 1.6 for the family $\left(G_{\omega}\right)_{\omega \in \Omega}$ of [68], Theorem 1.10 and its proof.

Section 5: The group of intermediate growth $\mathfrak{G}$, and the proof of Theorem 1.7; this is a quantitative sharpening of Theorem 1.6. Proposition 5.11 on $\mathrm{FP}_{2}$-covers of $\mathfrak{G}$.

- Appendix A: On soluble groups, metabelian groups, and finite presentations.

- Appendix B: On wreath products and lamplighter groups.

- Appendix C: On Bieri-Neumann-Strebel invariants.

- Appendix D: On growth and amenability. 


\section{Non-abelian free subgroups of finitely presented covers of contracting self-similar groups}

Let $G, H$ be two groups and $X$ a $H$-set; here, this means that $H$ acts on $X$ from the right. The corresponding permutational wreath product is the semi-direct product

$$
G \imath_{X} H:=G^{(X)} \rtimes H .
$$

We denote by $G^{(X)}$ the group of functions $\left(g_{x}\right)_{x \in X}: X \longrightarrow G, x \longmapsto g_{x}$ with finite support, and we consider the action from the left of $H$ on $G^{(X)}$, for which the action of $h$ on $\left(g_{x}\right)_{x \in X}$ is $\left(g_{x h}\right)_{x \in X}$. Hence the product of two elements in $G{ }_{2 X} H$ is given by

$$
\left(\left(g_{x}\right)_{x \in X}, h\right)\left(\left(g_{x}^{\prime}\right)_{x \in X}, h^{\prime}\right)=\left(\left(g_{x} g_{x h}^{\prime}\right)_{x \in X}, h h^{\prime}\right) .
$$

In case $G$ acts from the right on some set $W$, the group $G_{2 X} H$ acts naturally from the right on $W \times X$, by

$$
(w, y)\left(\left(g_{x}\right)_{x \in X}, h\right)=\left(w g_{y}, y h\right) .
$$

In expositions of wreath products and self-similar groups, choices of which groups actions are from the left and which from the right vary from one paper to the other, but a (sometimes hidden) mixture of left actions and right actions seems unavoidable.

If $G, G^{\prime}$ are two groups and $\alpha: G \longrightarrow G^{\prime}$ a homomorphism, we have a natural homomorphism

$$
\alpha^{2}{ }_{X} 1_{H}: G 2_{X} H \longrightarrow G^{\prime}{ }^{2} H
$$

where $1_{H}$ stands for the identity automorphism of $H$.

If $X$ is clear from the context, we write "" for " $X$ ". In particular, with $X=$ $\{0,1, \ldots, d-1\}$ and $S_{d}$ the full symmetric group of $X$, we write $G$ ? $S_{d}$ for $G \imath_{X} S_{d}$. Also, we write $1_{d}$ for $1_{S_{d}}$, and

$$
\left(\left(g_{x}\right)_{x \in X}, \tau\right)=\left(g_{0}, \ldots, g_{d-1}, \tau\right) \text { with } g_{0}, \ldots, g_{d-1} \in G \text { and } \tau \in S_{d}
$$

for a typical element of $G \imath S_{d}=G^{X} \rtimes S_{d}$.

The iterated wreath products with $S_{d}$ are defined inductively, for each integer $n \geq 0$, by

$$
G 2^{n} S_{d}= \begin{cases}G & \text { for } n=0 \\ \left(G 2^{n-1} S_{d}\right) \gtrless S_{d} & \text { for } n \geq 1\end{cases}
$$

We have the following associativity of permutational wreath products: for a $H$-set $X$ and a $K$-set $Y$, the canonical mapping

$$
\begin{cases}\left(G \imath_{X} H\right) \imath_{Y} K & \longrightarrow G 2_{X \times Y}\left(H 2_{Y} K\right) \\ \left(\left(\left(g_{x, y}\right)_{x \in X}, h_{y}\right)_{y \in Y}, k\right) & \longmapsto\left(\left(g_{x, y}\right)_{(x, y) \in X \times Y},\left(\left(h_{y}\right)_{y \in Y}, k\right)\right)\end{cases}
$$


is an isomorphism of groups (this is standard, see e.g. [115, Chapter 1, Theorem 3.2]). In particular, for $n \geq 1$, we have

$$
G \imath^{n} S_{d}=\left(G 2^{n-1} S_{d}\right) \imath S_{d}=\left(G \imath S_{d}\right) 2^{n-1} S_{d}=G^{X^{n}} \rtimes S_{d}^{(n)},
$$

where $S_{d}^{(n)}=\left(\cdots\left(S_{d}\right.\right.$ 々 $\left.\left.S_{d}\right) \imath \cdots\right)$ ₹ $S_{d}=S_{d}^{(n-1)}$ ₹ $S_{d}$ is the appropriate permutation group of $X^{n}$, acting here from the right. We write

$$
\left(\left(g_{v}\right)_{v \in X^{n}}, \tau\right) \text { with } g_{v} \in G \text { for all } v \in X^{n} \text { and } \tau \in S_{d}^{(n)}
$$

for a typical element of $G 2^{n} S_{d}$.

Definition 2.1 Let $G$ be a group and $d \geq 2$ an integer. A self-similar structure of degree $d$ on $G$ is a homomorphism

$$
\Phi: G \longrightarrow G \imath S_{d}
$$

A self-similar group is such a pair $(G, \Phi)$; when $\Phi$ is clear from the context, we write also " $G$ is a self-similar group".

If $(G, \Phi)$ is a self-similar group, the construction (1) gives rise to a sequence of homomorphisms

$$
\Phi_{n}: G \stackrel{\Phi_{n-1}}{\longrightarrow} G 2^{n-1} S_{d} \stackrel{\Phi<1}{\longrightarrow}\left(G<S_{d}\right) 2^{n-1} S_{d}=G \imath^{n} S_{d}
$$

for $n \geq 2$; we write $\Phi_{0}=\operatorname{id}_{G}$ and $\Phi_{1}=\Phi$. Note that, if $\Phi$ is injective, so is $\Phi_{n}$ for all $n \geq 0$. It is routine to check that $\Phi_{m+n}$ is the composition

$$
\Phi_{m+n}: G \stackrel{\Phi_{n}}{\longrightarrow} G 2^{n} S_{d} \stackrel{\Phi_{m} 21_{d}^{n}}{\longrightarrow}\left(G 2^{m} S_{d}\right) 2^{n} S_{d}=G 2^{m+n} S_{d}
$$

for all $m, n \geq 0$.

The composition of $\Phi_{n}$ and the quotient map $G \imath^{n} S_{d} \longrightarrow S_{d}^{(n)}$ is a homomorphism

$$
G \longrightarrow S_{d}^{(n)}, g \longmapsto \tau_{g}^{(n)}
$$

Thus, introducing the $v$-coordinates of $\Phi_{n}(\cdot)$, we have

$$
\Phi_{n}(g)=\left(\left(g_{v}\right)_{v \in X^{n}}, \tau_{g}^{(n)}\right) \in G 2 X^{n} S_{d}^{(n)}=G 2^{n} S_{d}
$$

for all $g \in G$. Note that

$$
\tau_{g}^{(n)}=\left(\left(\tau_{g_{x}}^{(n-1)}\right)_{x \in X}, \tau_{g}^{(1)}\right) \in S_{d}^{(n)}
$$

for all $g \in G$ and $n \geq 1$. 
Let $X^{*}=\bigsqcup_{n \geq 0} X^{n}$ be the free monoid over $X=\{0,1, \ldots, d-1\}$. The length of $v \in X^{*}$ is the integer $n=|v|$ such that $v \in X^{n}$. The $d$-regular rooted tree is the tree with vertex set $X^{*}$, and with edges connecting pairs of vertices of the form $\left(x_{1} \cdots x_{n}, x_{1} \cdots x_{n} x_{n+1}\right)$, with $n \geq 0$ and $x_{1}, \ldots, x_{n+1} \in X$; abusively, we denote this tree also by $X^{*}$. The homomorphisms of (5) define an action from the right of $G$ on the tree $X^{*}$.

For $n \geq 1$ and $v \in X^{n}$, we define the stabilizer of $v$ to be the subgroup

$$
\operatorname{Stab}_{G}(v)=\left\{g \in G \mid v \tau_{g}^{(n)}=v\right\}
$$

and we have a homomorphism

$$
\Phi_{v}: \operatorname{Stab}_{G}(v) \longrightarrow G, g \longmapsto g_{v}
$$

where $g_{v}=\Phi_{v}(g)$ is the $v$-coordinate of $\Phi_{n}(g)$.

Lemma 2.2 With the notation above,

$$
g_{v w}=\left(g_{v}\right)_{w},(g h)_{v}=g_{v} h_{v \tau_{g}^{(n)}}, \text { and }\left(h^{-1}\right)_{v}=\left(h_{v \tau_{h^{-1}}^{(n)}}\right)^{-1} \text {, }
$$

for all $g, h \in G, n \geq 1, v \in X^{n}$, and $w \in X^{*}$.

Proof To illustrate the fact that $G$ acts on $X^{*}$ from the right, we spell out the proof of the second equality, writing $v g$ for $v \tau_{g}^{(n)}$. We have on the one hand

$$
((v w) g) h=\left((v g)\left(w g_{v}\right)\right) h=(v g h)\left(w g_{v} h_{v g}\right)
$$

and on the other hand

$$
\left.(v w)(g h)=(v g h)\left(w(g h)_{v}\right)\right) .
$$

Hence $(g h)_{v}=g_{v} h_{v g}$.

Definition 2.3 A self-similar group $(G, \Phi)$ is faithful if its action on the tree $X^{*}$ described above is faithful; this implies that the homomorphism $\Phi$ is injective (but the converse does not hold $\left.{ }^{4}\right)$.

A self-similar group $(G, \Phi)$ is contracting if there is a finite subset $\mathcal{M} \subset G$ with the following property:

for all $g \in G$, there exists an integer $k \geq 0$

such that $g_{v} \in \mathcal{M}$ for all $v \in X^{*}$ with $|v| \geq k$.

\footnotetext{
${ }^{4}$ Set $H=\Phi^{-1}\left(G^{X}\right)$. The kernel of the action of $G$ on $X^{*}$ is the largest normal subgroup $N$ of $G$ that is contained in $H$ and such that $\Phi(N) \subset N^{X}$.
} 
The smallest such $\mathcal{M}$, namely

$$
\mathcal{N}:=\bigcup_{g \in G} \bigcap_{k \geq 0}\left\{g \in G \mid \exists h \in G, \ell \geq k, v \in X^{\ell} \text { with } h_{v}=g\right\}
$$

is called the nucleus of $(G, \Phi)$.

A self-similar group $(G, \Phi)$ is self-replicating ${ }^{5}$ if, for all $g \in G$ and $x \in X$, there exists $h \in \operatorname{Stab}_{G}(x)$ such that $h_{x}=g$, namely if, for all $x \in X$, the homomorphism $\Phi_{x}$ of (8) is onto. When this is so, it is easy to check by induction on the level that, for all $g \in G, n \geq 1$, and $v \in X^{n}$, there exists $h \in \operatorname{Stab}_{G}(v)$ such that $\Phi_{v}(h)=g$, namely $\Phi_{v}$ is onto.

Observe that, by definition, we have $1 \in \mathcal{N}$. Moreover for $g \in G$, we have $g \in \mathcal{N}$ if and only if $g^{-1} \in \mathcal{N}$, by Lemma 2.2.

The following proposition records basic facts about the nucleus of a contracting group.

Proposition 2.4 Let $(G, \Phi)$ be a contracting self-similar group with nucleus $\mathcal{N}$, as above.

(i) For $g \in \mathcal{N}$ and $x \in X$, we have $g_{x} \in \mathcal{N}$.

(ii) If $(G, \Phi)$ is self-replicating and $G$ is finitely generated, then $\mathcal{N}$ generates $G$.

Proof For $g \in \mathcal{N}$, there exist $h \in G, k \geq 0$, and $v \in X^{k}$ such that $h_{v}=g$ and $h_{w} \in \mathcal{N}$ for all $w \in X^{*}$ with $|w| \geq k$ (otherwise, $\mathcal{N}$ would not be minimal). Hence $g_{x}=\left(h_{v}\right)_{x}=h_{v x} \in \mathcal{N}$ for all $x \in X$. This proves (i).

For (ii), we paraphrase [118, Lemma 2.11.3]. Denote by $\langle\mathcal{N}\rangle$ the subgroup of $G$ generated by $\mathcal{N}$. Let $S$ be a symmetric finite generating set of $G$. For all $s \in S$, there exists $k_{s} \geq 0$ such that $s_{v} \in \mathcal{N}$ for all $v \in X^{*}$ with $|v| \geq k_{s}$. Set $k=\max \left\{k_{s} \mid s \in S\right\}$.

Let $g \in G$ and $v \in X^{*}$ with $|v| \geq k$. There exist $s_{1} \ldots, s_{m} \in S$ with $g=s_{1} \cdots s_{m}$, so that

$$
\begin{aligned}
g_{v} & =\left(s_{1}\right)_{v}\left(s_{2} \cdots s_{m}\right)_{v s_{1}}=\cdots \\
& =\left(s_{1}\right)_{v}\left(s_{2}\right)_{v s_{1}}\left(s_{3}\right)_{v s_{1} s_{2}} \cdots\left(s_{m}\right)_{v s_{1} \cdots s_{m-1}} \in\langle N\rangle,
\end{aligned}
$$

where the last inclusion follows from $|v|=\left|v s_{1}\right|=\cdots\left|v s_{1} \cdots s_{m-1}\right| \geq k$. In particular, the image of $\Phi_{v}$, as defined in (8), is contained in $\langle\mathcal{N}\rangle$.

If $(G, \Phi)$ is self replicating, then $\Phi_{v}$ is onto for all $v \in X^{*}$ with $|v| \geq 1$. The conclusion follows.

The next proposition was inspired to us by [118, Lemma 2.13.4]. We need some notation and a definition; our exposition borrows from [7].

Definition 2.5 Let $(G, \Phi)$ be a self-replicating contracting self-similar group, with nucleus $\mathcal{N}=\left\{n_{1}, \ldots, n_{\ell}\right\}$. Let $S=\left\{s_{1}, \ldots, s_{\ell}\right\}$ be a finite set given with a bijection

\footnotetext{
5 Or recurrent, or fractal, as in [118, Definition 2.8.1]. See [9] for relations of such groups with fractal sets.
} 
$s_{j} \leftrightarrow n_{j}$ with $\mathcal{N}$. Let $R$ be the set of relators in the letters of $S$ of one the forms

$$
\begin{array}{ll}
s_{i}=1 & \text { if } n_{i}=1 \in G, \\
s_{i} s_{j}=1 & \text { if } n_{i} n_{j}=1 \in G, \\
s_{i} s_{j} s_{k}=1 & \text { if } n_{i} n_{j} n_{k}=1 \in G .
\end{array}
$$

Note that these relators are of length at most 3; they are indexed by a subset of $\mathcal{N} \sqcup \mathcal{N}^{2} \sqcup \mathcal{N}^{3}$.

Assume furthermore that $G$ is finitely generated. The universal contracting cover of $G$ is the finitely presented group $G_{0}^{\text {un }}$ defined by the presentation with $S$ as set of generators and $R$ as set of relators. The assignment $\pi^{\text {un }}\left(s_{i}\right)=n_{i}$ extends to a group homomorphism

$$
\pi^{\mathrm{un}}: G_{0}^{\mathrm{un}}=\langle S \mid R\rangle \longrightarrow G,
$$

because $\pi^{\text {un }}(r)=1$ for any $r \in R$.

Note that $\pi^{\text {un }}$ is onto, by Proposition 2.4. We define finally

$$
\widehat{\pi}^{\mathrm{un}}=\pi^{\mathrm{un}} 21_{d}: G_{0}^{\mathrm{un}} 2 S_{d} \longrightarrow G 2 S_{d} \text {. }
$$

Remark 2.6 In particular examples, and for simplicity, it is often convenient to delete from $S$ the generator corresponding to $1 \in \mathcal{N}$, to delete $s_{k}$ if there exist $i, j \in\{1, \ldots, \ell\}$ with $n_{k}=n_{i} n_{j}$, and to delete one generator of every pair corresponding to $\left\{n, n^{-1}\right\} \subset$ $\mathcal{N}$. For example, in Example 2.17, we have $\mathcal{N}=\left\{1, a^{ \pm 1}, b^{ \pm 1}, c^{ \pm 1}\right\}$ with 7 elements, and $c=a^{-1} b$, but $S=\{a, b\}$ with 2 elements.

Note however that, in Example 2.16, we keep $d$ in the generating set $\{a, b, c, d\}$ of $G_{0}$, even though $d=b c$.

Proposition 2.7 Let $(G, \Phi)$ be a self-replicating contracting self-similar group of degree $d$, with nucleus $\mathcal{N}$. Assume that $G$ is finitely generated. Let $G_{0}^{\mathrm{un}}=\langle S \mid R\rangle$ and $\pi^{\mathrm{un}}: G_{0}^{\mathrm{un}} \rightarrow G$ be the universal contracting cover and the projection of Definition 2.5.

Then there exists a homomorphism

$$
\varphi_{1}^{\mathrm{un}}: G_{0}^{\mathrm{un}} \longrightarrow G_{0}^{\mathrm{un}} 2 S_{d}
$$

such that the self-similar group $\left(G_{0}^{\mathrm{un}}, \varphi_{1}^{\mathrm{un}}\right)$ is contracting, with nucleus $S$. Moreover the diagram

$$
\begin{array}{cc}
G_{0}^{\mathrm{un}} \stackrel{\varphi_{1}^{\mathrm{un}}}{\longrightarrow} G_{0}^{\mathrm{un}} & \imath S_{d} \\
\pi^{\mathrm{un}} \downarrow & \downarrow \quad \widehat{\pi}^{\mathrm{un}} \\
G & \stackrel{\Phi}{\longrightarrow} G \prec S_{d}
\end{array}
$$

commutes. 
Proof Step 1, definition of $\varphi_{1}^{\mathrm{un}}$. Denote by $\ell$ the order of $\mathcal{N}$, and write $\mathcal{N}=$ $\left\{n_{1}, \ldots, n_{\ell}\right\}$, as above. Let $i \in\{1, \ldots, \ell\}$. By Proposition 2.4, there exist $i_{0}, \ldots, i_{d-1} \in\{1, \ldots, \ell\}$ and $\tau_{i} \in S_{d}$ such that

$$
\Phi\left(n_{i}\right)=\left(n_{i_{0}}, \ldots, n_{i_{d-1}}, \tau_{i}\right) .
$$

We set

$$
\varphi_{1}^{\mathrm{un}}\left(s_{i}\right)=\left(s_{i_{0}}, \ldots, s_{i_{d-1}}, \tau_{i}\right) \in G_{0}^{\mathrm{un}} 2 S_{d}
$$

and we claim that this extends to a group homomorphism $\varphi_{1}^{\text {un }}$ as in (11).

Consider a relator as in Definition 2.5, say $s_{i} s_{j} s_{k}=1$ (shorter relators are dealt with similarly); hence $n_{i} n_{j} n_{k}=1 \in G$. Choose $x \in X$; recall that $X$ stands for $\{0, \ldots, d-1\}$. There exist $p, q, r \in\{1, \ldots, \ell\}$ and $\tau_{p}, \tau_{q}, \tau_{r} \in S_{d}$ such that the $x$-coordinate and the last coordinate of $\Phi\left(n_{i} n_{j} n_{k}\right)$ can be written as

$$
\left(n_{i} n_{j} n_{k}\right)_{x}=n_{p} n_{q} n_{r} \text { and } \tau_{n_{i} n_{j} n_{k}}=\tau_{p} \tau_{q} \tau_{r}
$$

Since $n_{i} n_{j} n_{k}=1 \in G$, we have

$$
n_{p} n_{q} n_{r}=1 \in G \forall x \in X \text { and } \tau_{p} \tau_{q} \tau_{r}=1 \in S_{d}
$$

Hence $\varphi_{1}^{\text {un }}\left(s_{i}\right) \varphi_{1}^{\text {un }}\left(s_{j}\right) \varphi_{1}^{\text {un }}\left(s_{k}\right)=1 \in G_{0}^{\text {un }}$. The claim is proven.

Step 2: $\left(G_{0}^{\mathrm{un}}, \varphi_{1}^{\mathrm{un}}\right)$ is a contracting group with nucleus $S$. For any word $w$ in the letters of $S$, we have to show that there exists a vertex $v \in X^{*}$ such that $(w)_{v} \in S$. By induction on the word length, and by Lemma 2.2, it is enough to show this for a word of length 2 .

Let $s_{i}, s_{j} \in S$ and $v \in X^{*}$ be such that $\left(n_{i} n_{j}\right)_{v} \in \mathcal{N}$, say $\left(n_{i} n_{j}\right)_{v}=n_{k}$. We have

$$
\left(n_{i}\right)_{v}\left(n_{j}\right)_{v \tau_{n_{i}}^{(|v|)}}=n_{k} \text { in } G
$$

which is a relator of length at most 3 . Hence the corresponding relator $\left(s_{i} s_{j}\right)_{v}=s_{k}$ holds in $S$.

It follows that $S$ is the nucleus of the group $\left(G, \varphi_{1}^{\text {un }}\right)$.

Step 3, commutativity of the diagram. This can be checked on the set $S$ of generators of $G_{0}^{\mathrm{un}}$.

The universal contracting cover $\left(G_{0}^{\mathrm{un}}, \varphi_{1}^{\mathrm{un}}\right)$ of $(G, \Phi)$ is uniquely defined by $(G, \Phi)$, and contracting. But we believe it need not be self-replicating (even though we do not know of any specific example). In all cases, $\left(G_{0}^{\text {un }}, \varphi_{1}^{\text {un }}\right)$ has quotients by finite sets of relations that are self-replicating contracting covers of $(G, \Phi)$, as described in the Definition 2.8 and Proposition 2.9. Note however that these quotients are no more uniquely defined by $(G, \Phi)$, since choices are involved.

In each of Examples 2.16 to 2.21 below, the universal contracting cover is selfreplicating. 
Definition 2.8 Let $(G, \Phi)$ be a self-replicating contracting self-similar group, with nucleus $\mathcal{N}=\left\{n_{1}, \ldots, n_{\ell}\right\}$; assume that $G$ is finitely generated. Let $S=\left\{s_{1}, \ldots, s_{\ell}\right\}$ be in bijection with $\mathcal{N}$, and $\left(G_{0}^{\mathrm{un}}, \varphi_{1}^{\mathrm{un}}\right)$ the universal contracting cover of $(G, \Phi)$, as in Definition 2.5. Let $\pi^{\text {un }}: G_{0}^{\text {un }} \longrightarrow G$ be as in (9).

Let $x \in X$ and $n_{i} \in \mathcal{N}$. Since the pair $(G, \Phi)$ is self-replicating, there exists ${ }^{6}$ $g\left(x, n_{i}\right) \in \operatorname{Stab}_{G}(x)$ such that $\left(g\left(x, n_{i}\right)\right)_{x}=n_{i}$. Since $\pi^{\text {un }}$ is onto, there exists $h\left(x, n_{i}\right) \in G_{0}^{\mathrm{un}}$ such that $\pi^{\mathrm{un}}\left(h\left(x, n_{i}\right)\right)=g\left(x, n_{i}\right)$; moreover, since $\widehat{\pi}^{\mathrm{un}}$ is the identity on the permutations of the wreath product, we have $h\left(x, n_{i}\right) \in \operatorname{Stab}_{G_{0}^{\mathrm{un}}}(x)$. By commutativity of Diagram (11), we have $\pi^{\mathrm{un}}\left(\left(h\left(x, n_{i}\right)\right)_{x}\right)=n_{i}$. Set $w\left(x, n_{i}\right)=$ $\left(h\left(x, n_{i}\right)\right)_{x} s_{i}^{-1}$; then $w\left(x, n_{i}\right)$ belongs to the kernel of $\pi^{\text {un }}$.

Again, by commutativity of (11), we have $\left(w\left(x, n_{i}\right)\right)_{v} \in \operatorname{ker}\left(\pi^{\text {un }}\right)$ for all $v \in X^{*}$. Since $\left(G_{0}^{\mathrm{un}}, \varphi_{1}^{\mathrm{un}}\right)$ is contracting, the subset

$$
E\left(x, n_{i}\right)=\left\{g \in G_{0}^{\mathrm{un}} \mid g=\left(w\left(x, n_{i}\right)\right)_{v} \text { for some } v \in X^{*}\right\}
$$

of $G_{0}^{\text {un }}$ is finite. Define

$$
E=\bigcup_{x \in X, n \in \mathcal{N}} E(x, n) \text { and } H=\langle\langle E\rangle\rangle \subset G_{0}^{\text {un }} \text {, }
$$

where $\langle\langle E\rangle\rangle$ denote the normal subgroup of $G_{0}^{\text {un }}$ generated by $E$.

A standard contracting cover of $G$ is a quotient group of the form $G_{0}=G_{0}^{\mathrm{un}} / H$, with $H$ as above; the image of $S$ in $G$ is a generating set, that we denote again (abusively) by $S$. Note that $E$ is a finite subset of $G_{0}^{\text {un }}$, and consequently that $G_{0}$ is a finitely presented group.

The epimorphism $\pi$ un factors through a homomorphism $\pi: G_{0} \longrightarrow G$, because $E$ is a subset of ker $\pi^{\text {un }}$. It follows from the definition that the restriction of $\pi$ to the generating set $S$ of $G_{0}$ is injective.

The following proposition is the analogue of proposition 2.7 for $G_{0}$.

Proposition 2.9 Let $(G, \Phi)$ be a self-replicating contracting self-similar group of degree d, with nucleus $\mathcal{N}$. Assume that $G$ is finitely generated. Let $G_{0}$ and $\pi: G_{0} \rightarrow G$ be a standard contracting cover of $(G, \Phi)$ and its projection to $G$, as in Definition 2.8.

Then there exists a homomorphism

$$
\varphi_{1}: G_{0} \longrightarrow G_{0} 2 S_{d}
$$

such that the self-similar group $\left(G_{0}, \varphi_{1}\right)$ is contracting and self-replicating with nucleus $S$. Moreover the diagram

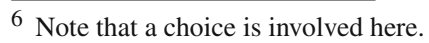




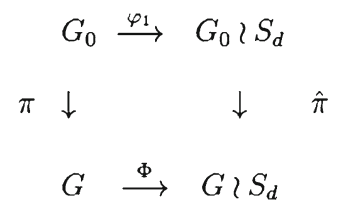

commutes.

Proof By construction of the set $E$, for any element $g \in E$ and any $x \in X$, we have $g_{x} \in E$. Hence the homomorphism $\varphi_{1}^{\text {un }}: G_{0}^{\text {un }} \longrightarrow G_{0}^{\text {un }} S_{d}$ induces a homomorphism $\varphi_{1}: G_{0} \longrightarrow G_{0} 2 S_{d}$. Since $\left(G_{0}^{\text {un }}, \varphi_{1}^{\text {un }}\right)$ is contracting with nucleus $S$, the self-similar group $\left(G_{0}, \varphi_{1}\right)$ is contracting with nucleus $S$.

Let $x \in X$, and $n_{i} \in \mathcal{N}$. We continue with the notation of Definition 2.8. By construction of $G_{0}$, the relation $h\left(x, n_{i}\right)=s_{i}$ holds in $G_{0}$; moreover $h\left(x, n_{i}\right)$ is an element of $\operatorname{Stab}_{G_{0}}(x)$. This shows that the pair $\left(G_{0}, \varphi_{1}\right)$ is self-replicating.

The commutativity of diagram (12) can be checked on the generators of $G_{0}$.

From here to Corollary 2.15, we keep the same notation as in Definition 2.8 and Proposition 2.9 for $G_{0}, \pi$, and $\varphi_{1}$, in relation with a given contracting self-replicating self-similar group $(G, \Phi)$, with $G$ finitely generated.

Definition 2.10 For an integer $n \geq 0$, define

(i) the homomorphism $\varphi_{n}: G_{0} \longrightarrow G_{0} 2^{n} S_{d}$ as in (3),

(ii) its kernel $N_{n}=\operatorname{ker}\left(\varphi_{n}\right)$ and the quotient $G_{n}=G_{0} / N_{n}$,

(iii) the homomorphism

$$
\widehat{\pi}_{n}=\pi \imath 1_{d^{n}}: G_{0} 2^{n} S_{d} \longrightarrow G 2^{n} S_{d}
$$

as in (1); note that $\widehat{\pi}_{1}$ is the $\widehat{\pi}$ of $(10)$.

We have $\Phi_{n} \pi=\widehat{\pi}_{n} \varphi_{n}$, i.e. the diagram

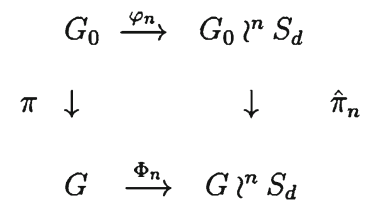

commutes. Observe that $N_{0} \subset \cdots \subset N_{n} \subset N_{n+1} \subset \cdots$ and define

$$
N=\bigcup_{n=0}^{\infty} N_{n} \text {. }
$$

Remark 2.11 As noted in Definition 2.8, the restriction of $\pi$ to $S$ is injective. More generally, in Definition 2.10, the restriction of $\widehat{\pi}_{n}$ to the subset $\left(S^{X^{n}}, 1\right)$ of $G_{0} 2^{n} S_{d}=$ $G_{0}^{X^{n}} \rtimes S_{d}^{(n)}$ is injective. 
Lemma 2.12 Let $(G, \Phi)$ be a self-similar group; assume that $G$ is finitely generated and that $(G, \Phi)$ is faithful contracting self-replicating. With the notation above, we have

$$
N=\operatorname{ker} \pi, \text { namely } G_{0} / N=G \text {, }
$$

so that

$$
\lim _{n \rightarrow \infty} G_{n}=G
$$

in the space of marked groups on $|S|$ generators (in the sense of Section 3).

Proof Let $g \in N$. Let $n \geq 1$ be such that $g \in \operatorname{ker}\left(\varphi_{n}\right)$. Then $\Phi_{n} \pi(g)=\widehat{\pi}_{n} \varphi_{n}(g)=1$, hence $g \in \operatorname{ker}(\pi)$ by the faithfulness assumption.

Conversely, let $k \in \operatorname{ker}(\pi)$. On the one hand, since $\left(G_{0}, \varphi\right)$ is contracting, there exists $n \geq 0$ such that $k_{v} \in S$ for all $v \in X^{n}$. On the other hand, $\pi(k)=1$ implies $\pi\left(k_{v}\right)=1$ for all $v \in X^{n}$; moreover, the $S_{d}^{(n)}$-coordinate of $\varphi_{n}(k)$ is 1 , by commutativity of Diagram (14). Hence, by Remark (2.11), we have $k_{v}=1$ for all $v \in X^{n}$, and therefore $k \in N_{n}=\operatorname{ker}\left(\varphi_{n}\right)$, a fortiori $k \in N$.

Lemma 2.13 In the situation of the previous lemma, for all $n \geq 1$, we have

$$
N_{n}=\varphi_{1}^{-1}\left(N_{n-1}^{d}\right)
$$

so that $\varphi_{1}: G_{0} \longrightarrow G_{0}$ 2 $S_{d}$ induces a homomorphism

$$
\psi_{n}:\left\{\begin{array}{l}
G_{n} \longrightarrow G_{n-1} \prec S_{d} \\
g N_{n} \longmapsto\left(\left(\varphi_{1}(g)_{x} N_{n-1}\right)_{x \in X}, \tau_{g}^{(1)}\right) .
\end{array}\right.
$$

Moreover $\psi_{n}$ is injective.

Proof For $g \in G$, write

$$
\varphi(g)=\left(\left(g_{x}\right)_{x \in X}, \tau_{g}^{(1)}\right) \quad \text { and } \quad \varphi_{n}(g)=\left(\left(g_{v}\right)_{v \in X^{n}}, \tau_{g}^{(n)}\right) .
$$

Assume first that $g \in N_{n}$. Thus $\left(g_{x}\right)_{v^{\prime}}=1$ and $\tau_{g_{x}}^{(n-1)}=1$ for all $x \in X$ and $v^{\prime} \in X^{n-1}$. This can be written

$$
\varphi_{n-1}\left(g_{x}\right)=\left(\left(\left(g_{x}\right)_{v^{\prime}}\right)_{v^{\prime} \in X^{n-1}}, \tau_{g_{x}}^{(n-1)}\right)=1 \forall x \in X,
$$

namely $g_{x} \in N_{n-1} \forall x \in X$. We have checked that $\varphi_{1}\left(N_{n}\right) \subset N_{n-1}^{d}$, and $N_{n} \subset$ $\varphi_{1}^{-1}\left(N_{n-1}^{d}\right)$ follows.

Assume now that $g \in \varphi_{1}^{-1}\left(N_{n-1}^{d}\right)$, namely that $\left(g_{x}\right)_{v^{\prime}}=1$ and $\tau_{g_{x}}^{(n-1)}=1$ for all $x \in X$ and $v^{\prime} \in X^{n-1}$. This can be written $g_{v}=1$ for all $v \in X^{n}$ and $\tau_{g}^{(n)}=1$, namely $g \in N_{n}$. Hence $\varphi_{1}^{-1}\left(N_{n-1}^{d}\right) \subset N_{n}$. 
The next theorem is a detailed version of Theorem 1.9.

Theorem 2.14 Let $(G, \Phi)$ be a self-similar group; assume that $G$ is finitely generated and that $(G, \Phi)$ is faithful contracting self-replicating. Let $G_{0}$ be a standard contracting cover, as in Definition 2.8 .

Assume that $G_{0}$ contains non-abelian free subgroups. Then, for each $n \geq 0$, the group $G_{n}$ of Definition 2.10 contains non-abelian free subgroups. More generally, every finitely presented cover of $G$ contains non-abelian free subgroups.

Assume moreover that $G_{0}$ is large. Then every finitely presented cover of $G$ is large.

Proof Let $S_{d}^{(0)}$ be the subgroup of $S_{d}$ of permutations fixing the letter $x=0$. For $n \geq 1$, let $H_{n}$ be the finite index subgroup of $G_{n}$ defined by

$$
H_{n}=\psi_{n}^{-1}\left(G_{n-1}^{\{0,1, \ldots, d-1\}} \rtimes S_{d}^{(0)}\right),
$$

where $\psi_{n}$ is as in Lemma 2.13. Projection onto the first coordinate (i.e. the coordinate $x=0$ )

$$
p_{n}^{(0)}: G_{n}^{\{0,1, \ldots, d-1\}} \rtimes S_{d}^{(0)} \longrightarrow G_{n}
$$

defined by

$$
p_{n}^{(0)}\left(\left(g_{x} N_{n}\right)_{x \in X}, \tau\right)=g_{0} N_{n}
$$

is a group homomorphism. It turns out that the composition

$$
q_{n}^{(0)}: H_{n} \stackrel{\psi_{n}}{\longrightarrow} \psi_{n}\left(H_{n}\right) \stackrel{p_{n-1}^{(0)}}{\longrightarrow} G_{n-1}
$$

defines a group homomorphism from $H_{n}$ to $G_{n-1}$.

Given a generator $s N_{n-1}$ of $G_{n-1}$ (where $s$ is a generator of $G_{0}$ ), using the selfreplicating property of $\left(G_{0}, \varphi_{1}\right)$, let $h \in S t_{G_{0}}(0)$ be such that $\varphi_{1}(h)_{0}=s$. It turns out that $q^{(0)}\left(h N_{n}\right)=s N_{n-1}$ which shows that $q_{n}^{(0)}$ is onto $G_{n-1}$. The conclusion is that for each $n \geq 1, G_{n}$ contains a finite index subgroup $H_{n}$ which maps onto $G_{n-1}$.

Therefore, if $G_{0}$ contains non-abelian free subgroups (respectively is large), by induction on $n$, each $G_{n}$ will contain non-abelian free subgroups (respectively will be large). Then by Lemma 2.12 and Corollary 3.4 below, every finitely presented cover of $G$ will contain non-abelian free subgroups (respectively will be large).

Corollary 2.15 Let $G$ be as in Theorem 2.14. If $G_{0}$ contains non-abelian free subgroups, then $G$ is infinitely presented.

Proof Since $G_{0}$ does contain non-abelian free subgroups, by assumption, and $G$ does not, by [119, Theorem 4.2], $G$ cannot be finitely presented, by the previous theorem. 
Consider integers $d, \ell \geq 1$, and a system of relations

$$
\left\{\begin{array}{l}
s_{1}=\left(\left(s_{1}\right)_{0}, \ldots,\left(s_{1}\right)_{d-1}\right) \tau_{1} \\
\ldots \ldots \\
s_{\ell}=\left(\left(s_{\ell}\right)_{0}, \ldots,\left(s_{\ell}\right)_{d-1}\right) \tau_{\ell}
\end{array}\right.
$$

with

$$
\begin{aligned}
\left(s_{j}\right)_{x} \in\left\{s_{1}, \ldots, s_{\ell}\right\} & \text { and } \tau_{j} \in S_{d} \\
\text { for } j \in\{1, \ldots, \ell\} & \text { and } x \in X=\{0, \ldots, d-1\} .
\end{aligned}
$$

By induction on $n \geq 0$, the system (16) defines a set $S$ of automorphisms of the $d$-regular rooted tree $X^{*}$, again denoted by $s_{1}, \ldots, s_{\ell}$. These generate a group $G=\langle S\rangle$ of automorphisms of the tree $X^{*}$, and (16) define a self-similarity structure $\Phi$ on $G$; thus $G$ is a self-similar group as in Definition 2.1, and moreover $G$ is faithful. The system (16) is often denoted by $\Phi$ again.

We review below some classical examples of self-similar groups defined this way.

Example 2.16 The 4-generated group $\mathfrak{G}=\langle a, b, c, d\rangle$ of Theorem 1.6 and [66,67] is a self-similar group of degree 2 , with $\Phi: \mathfrak{G} \longrightarrow \mathfrak{G}$ ? $C_{2}$ defined by

$$
\Phi(a)=(1,1) \tau, \Phi(b)=(a, c), \Phi(c)=(a, d), \Phi(d)=(1, b) .
$$

Here, $C_{2}=\{1, \tau\}$ denotes the cyclic group of order 2 (written $S_{2}$ in Definition 2.1). The self-similar group $(\mathfrak{G}, \Phi)$ is faithful, contracting, and self-replicating. The group $\mathfrak{G}$ is of intermediate growth.

The nucleus is

$$
\mathcal{N}=\{1, a, b, c, d\}
$$

The universal contracting cover of Definition 2.5 has the presentation

$$
G_{0}=\left\langle a, b, c, d \mid a^{2}, b^{2}, c^{2}, d^{2}, b c d\right\rangle \simeq C_{2} * V,
$$

where $C_{2}$ is now the group $\{1, a\}$ and $V$ the Klein Vierergruppe $\{1, b, c, d\}$, isomorphic to $C_{2} \times C_{2}$. The sign $\simeq$ indicates an isomorphism of groups. It can easily be checked that this cover is self-replicating, so that a cover as in Definition 2.8 is not needed here.

Proofs of these facts, and of other properties of $\mathfrak{G}$, can be found in [66], [93, Chapter VIII], or [118, Section 1.6], to quote some of the existing expositions only; see also our Section 5. The group $\mathfrak{G}$ can be viewed as the IMG of an orbifold version of the tent map $T:[0,1] \longrightarrow[0,1]$, defined by $T(x)=2 x$ for $x \leq 1 / 2$ and $T(x)=2-2 x$ for $x \geq 1 / 2$ [120, Section 5.3]. Here and below, "IMG" stands for "Iterated Monodromy Group" (see [118]).

Observe that $C_{2} * V$ is virtually a non-abelian free group, because it is a free product of finite groups, distinct from $C_{2} * C_{2}$ (see for example [136, Proposition 4 in Number 
I.1.3, p. 14]). It contains a free subgroup $F_{3}$ of index 8 ; one easy way to check this involves computing virtual Euler-Poincaré characteristics, as in [136, Section 1.8]: if

$$
F_{x}=\operatorname{ker}\left(p: C_{2} * V \stackrel{\text { canonical }}{\longrightarrow} C_{2} \times V\right),
$$

where $F_{x}$ stands for the free group of rank $x$, then

$$
\chi\left(C_{2} * V\right)=\frac{1}{2}+\frac{1}{4}-1=\frac{1}{\left[C_{2} * V: \operatorname{ker} p\right]} \chi\left(F_{x}\right)=\frac{1}{8}(1-x),
$$

and therefore $x=3$.

Hence Theorem 1.6 is a particular case of Theorem 2.14.

Example 2.17 The 2-generated Basilica group $\mathfrak{B}=\langle a, b\rangle$ is a self-similar group of degree 2, with homomorphism $\Phi: \mathfrak{B} \longrightarrow \mathfrak{B}$ ? $C_{2}$ defined by

$$
\Phi(a)=(b, 1) \tau, \Phi(b)=(a, 1) .
$$

The self-similar group $(\mathfrak{B}, \Phi)$ is faithful, contracting, and self-replicating. The group $\mathfrak{B}$ is of exponential growth.

The nucleus is

$$
\mathcal{N}=\left\{1, a^{ \pm 1}, b^{ \pm 1}, c^{ \pm 1}\right\}, \text { where } c=a^{-1} b .
$$

The universal contracting cover has the presentation

$$
G_{0}=\langle a, b \mid \emptyset\rangle \simeq F_{2}
$$

It is self-replicating.

The group $\mathfrak{B}$ has been introduced in $[80,81]$. The name "Basilica" was given by Mandelbrot to the Julia set of the quadratic transformation $z \longmapsto z^{2}-1$ of the complex plane, in honour of the Basilica Cattedrale Patriarcale di San Marco, and its reflection in Venetian waters [112, p. 254]. The group $\mathfrak{B}$ was identified as $\operatorname{IMG}\left(z^{2}-1\right)$ in [9, Theorem 5.8], ${ }^{7}$ and the group was named "Basilica" in $[10,101,118]$.

Our notation for $\Phi(a)$ and $\Phi(b)$ is essentially that of [118, p. 208]; the roles of $a$ and $b$ are exchanged in [80].

Again, Theorem 1.8 is a particular case of Theorem 2.14.

Incidentally, since $\mathfrak{B}$ is amenable (references in Appendix D), Theorem 1.8 shows that $\mathfrak{B}$ is not finitely presented. Since we could not find references for a direct proof the latter statement in the literature, let us allude to two other simple ways to show that $\mathfrak{B}$ is not finitely presented. One, suggested by Julia Bartsch (private communication), uses the infinite presentation of $\mathfrak{B}$ given in [81] and obtained together with Laurent Bartholdi; then a nice argument concludes that this presentation is minimal (erasing any of its relators would change the group). The other uses the contracting property of the Basilica group established in [80] and follows the idea indicated in [66] for $\mathfrak{G}$.

\footnotetext{
7 As acknowledged in [9], part of the credit for this is due to Richard Pink.
} 
Example 2.18 The IMG of $z^{2}+i$

$$
\mathfrak{J}=\operatorname{IMG}\left(z^{2}+i\right)=\langle a, b, c\rangle
$$

is defined by

$$
\Phi(a)=(1,1) \tau, \Phi(b)=(a, c), \Phi(c)=(b, 1) .
$$

It was studied in detail in [84], and was shown to be of intermediate growth in [40].

Its nucleus is

$$
\mathcal{N}=\{1, a, b, c\}
$$

and the only non-trivial relators of length $\leq 3$ among elements of $\mathcal{N}$ are $a^{2}=b^{2}=$ $c^{2}=1$ (this can best be checked with the GAP package (http://www.gap-system.org/ Packages/automgrp.html). Thus the universal contracting cover

$$
G_{0}=\left\langle a, b, c \mid a^{2}, b^{2}, c^{2}\right\rangle \simeq C_{2} * C_{2} * C_{2}
$$

has a free subgroup of finite index (indeed a subgroup $F_{5}$ of index 8 ). It is selfreplicating.

Example 2.19 The Gupta-Sidki group $\mathfrak{G} \mathfrak{S}=\langle a, b\rangle$ is the 2-generated group of automorphisms of the ternary rooted tree defined by

$$
\Phi(a)=(1,1,1) \tau, \Phi(b)=\left(a, a^{-1}, b\right),
$$

where $\tau \in S_{3}$ is the cyclic permutation $(0,1,2)$. It is the infinite 3 -group introduced in [88]; it is just infinite [8, Proposition 8.3]; it can be viewed as an IMG [120, Section 4.5].

Its nucleus is

$$
\mathcal{N}=\left\{1, a, a^{-1}, b, b^{-1}\right\}
$$

The universal contracting cover is

$$
G_{0}=\left\langle a, b \mid a^{3}, b^{3}\right\rangle \simeq C_{3} * C_{3},
$$

and contains a free subgroup $F_{4}$ of index 9. It is self-replicating.

The growth type of $\mathfrak{G} \mathfrak{S}$ is not known.

Example 2.20 The Fabrykowski-Gupta group $\mathfrak{F} \mathfrak{G}=\langle a, b\rangle$ is the 2-generated group of automorphisms of the ternary rooted tree defined by

$$
\Phi(a)=(1,1,1) \tau, \Phi(b)=(a, 1, b),
$$

with $\tau$ as in Example 2.19 [61,62]. It is of intermediate growth (see the original papers, and an exposition with improved estimates of growth in [12]), it is just infinite 
[8, Proposition 6.2], and it is the IMG of the cubic polynomial $z^{3}\left(-\frac{3}{2}+i \frac{\sqrt{3}}{2}\right)+1$ [120, Section 5.4].

As in the previous example, the nucleus is

$$
\mathcal{N}=\left\{1, a, a^{-1}, b, b^{-1}\right\}
$$

The universal contracting cover is

$$
G_{0}=\left\langle a, b \mid a^{3}, b^{3}\right\rangle \simeq C_{3} * C_{3} .
$$

It is self-replicating.

Example 2.21 The ternary Hanoi Towers group $\mathfrak{H}=\langle a, b, c\rangle$ is the 3-generated group of automorphisms of the ternary rooted tree defined by

$$
\Phi(a)=(a, 1,1) \tau_{1,2}, \Phi(b)=(1, b, 1) \tau_{0,2}, \Phi(c)=(1,1, c) \tau_{0,1}
$$

where $\tau_{1,2}$ is the transposition of $S_{3}$ exchanging 1 and 2 , and similarly for $\tau_{0,2}, \tau_{0,1}$. It was introduced in [82] as a model for the well-known Hanoi Towers problem; it is known to be of exponential growth ([83, Subsection 6.1] and [74]), and isomorphic to $\operatorname{IMG}\left(z^{2}-\frac{16}{27 z}\right)$ [83, Example 8].

The nucleus is

$$
\mathcal{N}=\{1, a, b, c\}
$$

The universal contracting cover is

$$
G_{0}=\left\langle a, b, c \mid a^{2}, b^{2}, c^{2}\right\rangle \simeq C_{2} * C_{2} * C_{2} .
$$

It is self-replicating.

Theorem 2.22 Any finitely presented cover of one of the groups $\mathfrak{J}, \mathfrak{G} \mathfrak{S}, \mathfrak{F} \mathfrak{G}, \mathfrak{H}$, of the four previous examples is large.

This is a straightforward consequence of Theorem 2.14. In Section 4, we will show how to modify 2.14 to cover uncountably many examples.

Remark 2.23 Groups of interest here are often known to have rather few quotients, of special kinds. Let us illustrate this as follows.

(i) A group is just infinite if all its proper quotients are finite. The group $\mathfrak{G}$ is just infinite. More generally, with the notation of section 4 , the group $G_{\omega}$ is just infinite for all $\omega \in \Omega_{0}$ (as we repeat below in Proposition 4.2.ii).

(ii) Without recalling here the technical definitions, let us mention the following property of a finitely generated group $G$ assumed to be branch, or even weakly branch: for any normal subgroup $N \neq\{1\}$ of $G$, there exists an integer $n \geq 1$ such that $N$ contains the derived group of the rigid stabilizer $\operatorname{Rist}_{G}(n)$; this follows from the proof of [72, Theorem 4]. 
As a consequence, if $G$ is branch, then any proper quotient of $G$ is virtually abelian. In particular, any proper quotient of one of the groups $\mathfrak{J}, \mathfrak{G} \mathfrak{S}, \mathfrak{F} \mathfrak{G}$, and $\mathfrak{H}$, is virtually abelian.

(This does not apply to $\mathfrak{B}$, which is weakly branch but not branch group. This applies to $\mathfrak{G}$, but it is of little interest in this case since the property of (i) is strictly stronger.)

(iii) It is shown in [70] that $\mathfrak{G}$ has a finitely presented HNN-extension $\widehat{\mathfrak{G}}$ which is in $\mathcal{S G} \backslash \mathcal{E} \mathcal{G}$. Any proper quotient of $\widehat{\mathfrak{G}}$ is metabelian and virtually abelian [133, Theorem 2.3].

(iv) The Basilica group $\mathfrak{B}$ is just non-soluble, which means that all its proper quotients are soluble [80, Proposition 6].

(v) Recall however that there exist groups of intermediate growth with uncountably many quotients: see [69] and Definition 4.13.

\section{Marked groups and the Chabauty topology}

For $k$ a positive integer, let $F_{k}$ denote the free group of rank $k$, given together with an ordered free basis $\left(s_{1}, \ldots, s_{k}\right)$ of generators. A marked group of rank $k$ is a pair $(G, S)$ where $G$ is a group and $S$ an ordered set of $k$ generators (for distinct $s, t \in S$, equalities $s=1$ and $s=t \in G$ are allowed). To such a pair corresponds a free cover $\pi_{G}: F_{k} \rightarrow G$, with $\pi_{G}\left(s_{j}\right)$ being the $j$ th generator of $S(1 \leq j \leq k)$. We denote by $\mathcal{M}_{k}$ the set of marked groups on $k$ generators, identified here with the set of normal subgroups of $F_{k}$ via the bijection $(G, S) \longleftrightarrow \operatorname{ker} \pi_{G}$.

The idea to furnish a space of (sub)groups with a topology goes back at least to Chabauty [45], and has been revisited on many occasions, among others by Bourbaki [36, chapitre VIII, § 5], Gromov [87, final remarks], one of us [68], Stepin [144], Champetier [46], Champetier and Guirardel [47], and Ceccherini-Silberstein and Coornaert [43].

The Chabauty topology on $\mathcal{M}_{k}$, also called the Cayley topology, is that defined by the basis ${ }^{8}$

$$
\mathcal{O}_{K, K^{\prime}}=\left\{N \triangleleft F_{k}: N \cap K=\emptyset \text { and } K^{\prime} \subset N\right\}
$$

with $K, K^{\prime}$ finite subsets in $F_{k}$. This topology makes $\mathcal{M}_{k}$ a totally disconnected compact space. It is also completely metrisable, as we now recall. For two subsets $A, A^{\prime}$ in $F_{k}$, let $v\left(A, A^{\prime}\right)$ denote the largest integer $n$ such that $A \cap B_{S}^{F_{k}}(n)=A^{\prime} \cap B_{S}^{F_{k}}(n)$, where $S=\left(s_{1}, \ldots, s_{k}\right)$ in $F_{k}$ is as above, and where balls $B_{S}^{F_{k}}(n)$ are as in Appendix D. Set $d\left(A, A^{\prime}\right)=\exp \left(-v\left(A, A^{\prime}\right)\right)$. Then $d$ is a metric (indeed an ultrametric) and makes the set $2^{F_{k}}$ of subsets of $F_{k}$ a totally discontinuous compact metric space, in

8 There is an equivalent definition in terms of the subbasis

$$
\mathcal{O}_{K, V}=\left\{N \triangleleft F_{k}: N \cap K=\emptyset \text { and } N \cap V \neq \emptyset\right\},
$$

indexed by pairs $(K, V)$ where $K$ is a finite subset of $F_{k}$ and $V$ a subset of $F_{k}$. With $K$ compact and $V$ open, it has the advantage to carry over to the space of closed subgroups of a locally compact group $G$. 
which the space $\mathcal{M}_{k}$ of marked groups on $k$ generators (namely the space of normal subgroups of $F_{k}$ ) is closed. The topology induced by $d$ on $\mathcal{M}_{k}$ coincides with that defined by (17).

Here is an elementary and basic fact about this topology. The earliest written reference we know for it is [53, Lemma 1.3 and Lemma 1].

Proposition 3.1 Let $k, \ell$ be two positive integers. Let $(G, S) \in \mathcal{M}_{k}$ and $(G, T) \in \mathcal{M}_{\ell}$ be two marked groups with the same underlying group. Then there exist neighbourhoods $U \subset \mathcal{M}_{k}$ of $(G, S)$ and $V \subset \mathcal{M}_{\ell}$ of $(G, T)$ that are homeomorphic.

In loose words, local properties of $(G, S)$ are properties of $G$ itself.

This proposition justifies the following definitions: a property $(\mathcal{P})$ of finitely generated groups is open [respectively closed] if, for any positive integer $k$, the subset of $\mathcal{M}_{k}$ of marked groups $(G, S)$ such that $G$ has Property $(\mathcal{P})$ is open [respectively closed]. A finitely generated group $G$ is isolated if, for any ordered generating set $S=\left(s_{1}, \ldots, s_{k}\right)$ of $G$, the point $(G, S)$ is isolated in $\mathcal{M}_{k}$. We collect a few examples as follows:

Proposition 3.2 For $k \geq 2$, in the space $\mathcal{M}_{k}$ of marked groups of rank $k$ :

(i) "Being abelian" is both an open and a closed property; more generally, for $d \geq 1$, "being nilpotent of nilpotent class at most $d$ " is both open and closed. "Being nilpotent" is open and non-closed.

(ii) "Being soluble of solubility class at most $k$ " is closed and non-open. "Being soluble" is neither open nor closed.

(iii) "Being finite" and "having torsion" are open and non-closed.

(iv) If $(G, S) \in \mathcal{M}_{k}$ is a marking of a finitely presented group $G$, there exists a neighbourhood of $(G, S)$ in $\mathcal{M}_{k}$ containing only marked quotients of $(G, S)$.

(v) A necessary condition for $(G, S)$ to be an isolated point in $\mathcal{M}_{k}$ is that $G$ is finitely presented. Finite groups and finitely presented simple groups are isolated.

(vi) There exists an isolated group that is 3-soluble and non-Hopfian; the group $\widehat{\mathfrak{G}}$ mentioned in Remark 2.23.iii is isolated.

(vii) Amenability is neither open nor closed.

(viii) Kazhdan Property (T) is open in $\mathcal{M}_{k}$.

(ix) Serre Property (FA) is not open in $\mathcal{M}_{k}$.

On the proof Claims (i) to (v) are elementary; most of them appear explicitly in [47, Section 2.6 and Lemma 2.3]. For (i), note moreover that "being nilpotent" is open by (iv), because nilpotent groups are finitely presented. For (ii), note that "being soluble" is non-open, because metabelian groups like $\mathbf{Z} \imath \mathbf{Z}$ are limits of non-soluble groups (see Example B.2, say).

"Being nilpotent", "being soluble", "being finite", "being amenable" and "having torsion" are non-closed properties, because non-abelian free groups are residually finite $p$-groups, for any prime $p$ (due to [89], see also [149]).

For (v), observe that a finitely generated infinitely presented group $G$ is always a limit of finitely presented groups $G_{n}$; more precisely

$$
\begin{aligned}
& G=\left\langle s_{1}, \ldots, s_{k} \mid\left(r_{i}\right)_{i \geq 1}\right\rangle=\lim _{n \rightarrow \infty} G_{n} \\
& \text { with } G_{n}=\left\langle s_{1}, \ldots, s_{k} \mid r_{1}, \ldots, r_{n}\right\rangle .
\end{aligned}
$$


Necessary and sufficient conditions for isolated points are known in terms of the existence of "finite discriminating subsets"; we refer to [53, Proposition 2]; see also [73, Theorem 2.1]. The class of isolated groups contains considerably more groups than the finite groups and the finitely presented simple groups [53].

The first part of Claim (vi) is [53, Proposition 10]; the second part is implicit in [133], and explicit in [53, Proposition 5.18]). "Being amenable" is non-open, again because $\mathbf{Z} \imath \mathbf{Z}$ is a limit of groups with non-abelian free subgroups (Example B.2). Claim (viii) is a result of [137], and (ix) of [57].

The Chabauty topology on $\mathcal{M}_{k}$ plays an important role in connection with many group properties including "Property LEF" and "soficity". The two latter properties define subspaces in $\mathcal{M}_{k}$ that are closed [43, Propositions 7.3.7 and 7.5.13].

Note the contraposition of (v): for $(G, S) \in \mathcal{M}_{k}$ with $G$ infinitely presented, there exists a sequence $\left(\left(G_{n}, S_{n}\right)\right)_{n \geq 1}$ of pairwise distinct points in $\mathcal{M}_{k}$ such that $\lim _{n \rightarrow \infty}\left(G_{n}, S_{n}\right)=(G, S)$.

The simplest examples of converging sequences in $\mathcal{M}_{k}$ are of the following kind. Let

$$
N_{1} \subset \cdots \subset N_{n} \subset N_{n+1} \subset \cdots \subset N:=\bigcup_{n \geq 1} N_{n}
$$

be a nested sequence of normal subgroups in $F_{k}$. Let $S_{0}$ be a free basis of $F_{k}$. Denote by $p_{n}: F_{k} \longrightarrow G_{n}:=F_{k} / N_{n}(n \geq 1)$ and $p: F_{k} \longrightarrow G:=F_{k} / N$ the canonical projections. Set $S_{n}=p_{n}\left(S_{0}\right)$ and $S=p\left(S_{0}\right)$. Then $\left(\left(G_{n}, S_{n}\right)\right)_{n \geq 1}$ is a sequence in $\mathcal{M}_{k}$ converging to $(G, S)$. In this case we often suppress the emphasis on the generating sets and write simply that the sequence $\left(G_{n}\right)_{n \geq 1}$ converges to $G$ in $\mathcal{M}_{k}$.

Converging sequences in $\mathcal{M}_{k}$ need not be of this special kind, with $G$ a quotient of $G_{n}$ for all $n$ large enough. See below, Proposition 4.2.vi.

The following observation about $\mathcal{M}_{k}$ and covers, basic for us, is well-known; see e.g. [54, Proposition 3.3]. We provide a proof for the convenience of the reader.

Proposition 3.3 Let $\left(\left(G_{n}, S_{n}\right)\right)_{n \geq 1}$ be a converging sequence in $\mathcal{M}_{k}$; set $(G, S)=$ $\lim _{n \rightarrow \infty}\left(G_{n}, S_{n}\right)$. Let $\Gamma$ be a finitely presented group; assume there exists a cover $\pi: \Gamma \rightarrow G$.

Then $\Gamma$ is a cover of $G_{n}$ for $n$ large enough.

Note. In case $G$ itself is finitely presented, this lemma is an immediate consequence of Proposition 3.2.v.

Proof Denote as above by $\left(s_{1}, \ldots, s_{k}\right)$ an ordered free basis of $F_{k}$. Let $p_{n}: F_{k} \rightarrow G_{n}$ and $p: F_{k} \rightarrow G$ be the free covers corresponding to $\left(G_{n}, S_{n}\right)$ and $(G, S)$ respectively. Set $N_{n}=\operatorname{ker}\left(p_{n}\right)$ and $N=\operatorname{ker}(p)$. Let $\left(t_{1}, \ldots, t_{\ell}\right)$ an ordered generating set of $\Gamma$. Consider the free group $F_{\ell}$ on an ordered basis $U=\left(u_{1}, \ldots, u_{\ell}\right)$ and the free cover $q: F_{\ell} \rightarrow \Gamma$ defined by $q\left(u_{j}\right)=t_{j}$ for $j=1, \ldots, \ell$.

Since $\Gamma$ is finitely presented, there exists a finite subset $R \subset F_{\ell}$ of words $v_{1}, \ldots, v_{m}$ in the letters of $U \cup U^{-1}$ such that $\operatorname{ker}(q)$ is the normal subgroup of $F_{\ell}$ generated by $R$, namely such that $\langle U \mid R\rangle$ is a presentation of $\Gamma$. For $j \in\{1, \ldots, \ell\}$, choose a word 
$w_{j}$ in the letters $p\left(s_{1}\right), \ldots, p\left(s_{k}\right)$ and their inverses such that $\pi\left(t_{j}\right)=w_{j}$. Let $\tilde{w}_{j}$ be the word in $\left\{s_{1}, s_{1}^{-1}, \ldots, s_{k}, s_{k}^{-1}\right\}$ obtained by substitution of $s_{i}^{ \pm 1}$ in place of $p\left(s_{i}\right)^{ \pm 1}$; observe that $p\left(\tilde{w}_{j}\right)=w_{j}=\pi\left(t_{j}\right)$. Consider the homomorphism

$$
h: F_{\ell} \longrightarrow F_{k} \quad \text { defined by } \quad h\left(u_{j}\right)=\tilde{w}_{j} \quad(1 \leq j \leq \ell) .
$$

Then $p h\left(u_{j}\right)=p\left(\tilde{w}_{j}\right)=w_{j}=\pi\left(t_{j}\right)=\pi q\left(u_{j}\right)$ for all $j$, so that $p h=\pi q$, and therefore $h(R) \subset N$.

The last inclusion means that the open subset

$$
\mathcal{O}^{\prime}:=\left\{M \triangleleft F_{k}: h(R) \subset M\right\}=\bigcap_{i=1}^{m} \mathcal{O}_{\emptyset,\left\{h\left(r_{i}\right)\right\}}
$$

is a neighbourhood of $N$ in $\mathcal{M}_{k}$. Hence, for $n$ large enough, we have $N_{n} \in \mathcal{O}^{\prime}$ and therefore $h(R) \subset N_{n}$.

Denote by $\langle\langle T\rangle\rangle$ the normal subgroup of a group $H$ generated by a subset $T \subset H$. Let

$$
h_{1}: \Gamma=F_{\ell} /\langle\langle R\rangle\rangle \longrightarrow F_{k} /\langle\langle h(R)\rangle\rangle
$$

be the cover induced by $h$, and

$$
h_{2}: F_{k} /\langle\langle h(R)\rangle\rangle \longrightarrow F_{k} / N_{n}=G_{n}
$$

that defined by the inclusion $\langle\langle h(R)\rangle\rangle \subset N_{n}$ (for $n \gg 1$ ). The composition $h_{2} h_{1}$ is a cover $\Gamma \rightarrow G_{n}$, and this concludes the proof.

An immediate consequence of the previous proposition is the following corollary, of very frequent use in our work.

Corollary 3.4 Consider the three following group properties:

(NA) non-amenability,

(Fr) containing non-abelian free groups,

(La) being large.

Let $k \geq 2$ and $\left(\left(G_{n}, S_{n}\right)\right)_{n \geq 1}$ be a converging sequence in $\mathcal{M}_{k}$, with limit $(G, S)$.

If, for all $n$ large enough, $G_{n}$ has one of the three properties above, then any finitely presented cover of $G$ has the same property.

Proof The point is that a group that has a quotient with one of the properties (NA), (Fr), (La) has itself the same property.

\section{The analogue of Theorem 1.6 for the family $\left(G_{\omega}\right)_{\omega \in \Omega}$ of [68]}

Let $\Omega$ be the Cantor space $\{0,1,2\}^{\mathbf{N}}$ of all sequences of 0 's, 1 's and 2's, with the product topology. Denote by $\Omega_{-}$the countable subspace of eventually constant sequences, 
by $\Omega_{+}$its complement, and by $\Omega_{0}$ the subspace of sequences with infinitely many occurrences of each of $0,1,2$; thus

$$
\Omega_{0} \subset \Omega_{+} \subset \Omega=\Omega_{+} \sqcup \Omega_{-}
$$

We denote by $\sigma$ the shift on $\Omega$, defined by $(\sigma(\omega))_{n}=\omega_{n+1}$ for all $n \geq 1$.

We will recall the construction of [68], which is a generalisation of that of Section 2. It associates with each point $\omega \in \Omega$ a marked group $\left(G_{\omega}, S_{\omega}\right)$ with $S_{\omega}$ consisting of 4 generators of order 2; for example, $\mathfrak{G}=G_{\overline{012}}$, where $\overline{012}$ stands for the 3-periodic sequence $012012012 \cdots$. In this section, set

$$
X=\{0,1\}
$$

and identify $X^{*}$ with the 2-regular rooted tree. We proceed to define for all $\omega \in \Omega$ a marked group $\left(G_{\omega} . S_{\omega}\right) \in \mathcal{M}_{4}$ of automorphisms of $X^{*}$.

Definition 4.1 The flip $a \in \operatorname{Aut}\left(X^{*}\right)$ is defined by

$$
a(0 v)=1 v \text { and } a(1 v)=0 v \text { for all } v \in X^{*} .
$$

Set

$$
\begin{array}{lll}
a_{\beta(0)}=a & a_{\beta(1)}=a & a_{\beta(2)}=1 \\
a_{\gamma(0)}=a & a_{\gamma(1)}=1 & a_{\gamma(2)}=a \\
a_{\delta(0)}=1 & a_{\delta(1)}=a & a_{\delta(2)}=a
\end{array}
$$

Define for each $\omega=\left(\omega_{n}\right)_{n \geq 1} \in \Omega$ a set $S_{\omega}=\left\{a, b_{\omega}, c_{\omega}, d_{\omega}\right\}$ of four automorphisms of $X^{*}$ by

$$
\begin{aligned}
& b_{\omega}=\left(a_{\beta\left(\omega_{1}\right)}, b_{\sigma(\omega)}\right) \\
& c_{\omega}=\left(a_{\gamma\left(\omega_{1}\right)}, c_{\sigma(\omega)}\right) \\
& d_{\omega}=\left(a_{\delta\left(\omega_{1}\right)}, d_{\sigma(\omega)}\right) .
\end{aligned}
$$

It is easy to check that

$$
\begin{aligned}
a c_{\omega} a & =\left(b_{\sigma(\omega)}, a_{\beta\left(\omega_{1}\right)}\right) \\
a d_{\omega} a & =\left(c_{\sigma(\omega)}, a_{\gamma\left(\omega_{1}\right)}\right) \\
a b_{\omega} a & =\left(d_{\sigma(\omega)}, a_{\delta\left(\omega_{1}\right)}\right) \\
a^{2}=b_{\omega}^{2} & =c_{\omega}^{2}=d_{\omega}^{2}=b_{\omega} c_{\omega} d_{\omega}=1 .
\end{aligned}
$$

We define the group

$$
G_{\omega}=\left\langle a, b_{\omega}, c_{\omega}, d_{\omega}\right\rangle
$$

generated by $S_{\omega}$; it is a subgroup of $\operatorname{Aut}\left(X^{*}\right)$. It follows from the last line of (18) that any element of $G_{\omega}$ can be written as 


$$
(*) a * a * \cdots a(*)
$$

with $* \in\left\{b_{\omega}, c_{\omega}, d_{\omega}\right\},(*) \in\left\{1, b_{\omega}, c_{\omega}, d_{\omega}\right\}$, and $n \geq 0$ occurrences of $a$.

Observe that any permutation $\tau$ of $\{0,1,2\}$ induces a permutation of $\Omega$, again denoted by $\tau$; the groups $G_{\tau(\omega)}$ and $G_{\omega}$ are isomorphic.

In [68, Section 6], there is moreover a modified construction providing a marked group $\left(\widetilde{G}_{\omega}, \widetilde{S}_{\omega}\right)$; we refer to the original paper. Note that (v) below holds for the modified groups, but not for the groups $G_{\omega}$.

Proposition 4.2 Let $\Omega=\{0,1,2\}^{\mathbf{N}}$. For $\omega \in \Omega$, let $G_{\omega}$ and $\widetilde{G}_{\omega}$ be as above.

(i) For $\omega \in \Omega$, the groups $G_{\omega}$ and $\widetilde{G}_{\omega}$ are both infinite, and $\widetilde{G}_{\omega}$ is infinitely presented.

(ii) For $\omega \in \Omega_{+}$, the marked groups $\left(G_{\omega}, S_{\omega}\right)$ and $\left(\widetilde{G}_{\omega}, \widetilde{S}_{\omega}\right)$ are isomorphic; the group $G_{\omega}$ is of intermediate growth.

For $\omega \in \Omega_{0}$, the group $G_{\omega}$ is an infinite 2-group, and is just infinite. ${ }^{9}$

(iii) For $\omega \in \Omega_{-}$, the group $G_{\omega}$ is virtually free abelian, and consequently finitely presented of polynomial growth, while the group $\widetilde{G}_{\omega}$ is virtually metabelian and of exponential growth.

(iv) For $\omega, \omega^{\prime} \in \Omega_{+}$, the groups $G_{\omega}$ and $G_{\omega^{\prime}}$ are isomorphic if and only if $\omega^{\prime}=\tau(\omega)$ for some permutation $\tau$ of $\{0,1,2\}$.

(v) The mapping $\Omega \longrightarrow \mathcal{M}_{4}, \omega \longmapsto\left(\widetilde{G}_{\omega}, \widetilde{S}_{\omega}\right)$ is a homeomorphism onto its image.

(vi) For a converging sequence $\left(\omega_{(n)}\right)_{n \geq 1}$ of points in $\Omega_{+}$with a limit $\omega$ in $\Omega_{+}$, we have $\lim _{n \rightarrow \infty}\left(G_{\omega_{(n)}}, S_{\omega_{(n)}}\right)=\left(G_{\omega}, S_{\omega}\right)$ in $\mathcal{M}_{4}$. If, moreover, $\omega_{(n)} \in \Omega_{0}$ for all $n \geq 1$ and $\omega \notin \Omega_{0}$, then, for all $n \geq 1$, the group $G_{\omega}$ is not a quotient of $G_{\omega_{(n)}}$.

On the proof Most of this is proved in [68]; more precisely:

(i) $G_{\omega}$ is infinite $[68$, Theorem 2.1$]$ and $\widetilde{G}_{\omega}$ is infinitely presented [68, Theorem 6.2].

(ii) For $\omega \in \Omega_{+}$, we have $\left(G_{\omega}, S_{\omega}\right)=\left(\widetilde{G}_{\omega}, \widetilde{S}_{\omega}\right)[68$, observation just before Theorem 6.1], and $G_{\omega}$ is of intermediate growth [68, Corollary 3.2]. For $\omega \in \Omega_{0}, G_{\omega}$ is an infinite 2-group that is just infinite [68, Theorems 2.1 and 8.1].

(iii) $G_{\omega}$ is virtually free abelian [68, Theorem 2.1.(3)], while $\widetilde{G}_{\omega}$ is virtually metabelian and of exponential growth [68, Theorem 6.1].

About (iv), see [118, Theorem 2.10.13]. A weaker statement is proved in [68, Section 5].

For (v), see [68, Proposition 6.2].

For (vi), given any $n \geq 1$, note that $G_{\omega}$ is neither isomorphic to $G_{\omega_{(n)}}$, by (iv), nor a non-trivial quotient of $G_{\omega_{(n)}}$, by (ii).

For the main result of this section (Theorem 4.5), we will need an analogue in the present context of the homomorphisms (2) and (3) of Section 2. Recall that we have

\footnotetext{
${ }^{9}$ Let $\Omega_{1}$ be the subset of $\Omega_{+}$of sequences containing infinitely many occurrences of two of $0,1,2$, and finitely many occurrences of the third, so that $\Omega_{+}=\Omega_{0} \sqcup \Omega_{1}$. For $\omega \in \Omega_{1}$, the group $G_{\omega}$ is not a 2-group, indeed it has elements of infinite order.
} 
a natural isomorphism

$$
\Phi_{X}: \operatorname{Aut}\left(X^{*}\right) \stackrel{\simeq}{\longrightarrow} \operatorname{Aut}\left(X^{*}\right) \gtrless S_{2}
$$

We keep the notation of Definition 4.1.

Definition 4.3 Let $\omega \in \Omega$. The restriction to $G_{\omega}$ of the isomorphism $\Phi_{X}$ provides an injective homomorphism

$$
\Phi_{\omega}^{(1)}=\Phi_{\omega}: G_{\omega} \longrightarrow G_{\sigma(\omega)} \prec S_{2}
$$

On the generators, we have

$$
\begin{aligned}
\Phi_{\omega}(a) & =(1,1) \tau \\
\Phi_{\omega}\left(b_{\omega}\right) & =\left(a_{\beta\left(\omega_{2}\right)}, b_{\sigma(\omega)}\right) \\
\Phi_{\omega}\left(c_{\omega}\right) & =\left(a_{\gamma\left(\omega_{2}\right)}, c_{\sigma(\omega)}\right) \\
\Phi_{\omega}\left(d_{\omega}\right) & =\left(a_{\delta\left(\omega_{2}\right)}, d_{\sigma(\omega)}\right)
\end{aligned}
$$

(recall that $S_{2}=\{1, \tau\}$ ). The sequence of homomorphisms $\left(\Phi_{\omega}^{(n)}\right)_{n \geq 1}$ is defined inductively by

$$
\Phi_{\omega}^{(n)}: G_{\omega} \stackrel{\Phi_{\omega}^{(n-1)}}{\longrightarrow} G_{\sigma^{n-1}(\omega)} 2^{n-1} S_{2} \stackrel{\Phi_{\sigma^{n-1}(\omega)}^{(1)} d^{n-1}}{\longrightarrow} G_{\sigma^{n}(\omega) 2^{n}} S_{2}
$$

Lemma 4.4 (contraction in $G_{\omega}$ ) Let $\omega \in \Omega$. We keep the notation above.

(i) For each $n \geq 1$, the homomorphism $\Phi_{\omega}^{(n)}$ is injective.

(ii) For all $g \in G_{\omega}$, there exists an integer $n \geq 1$ such that

$$
\Phi_{\omega}^{(n)}(g)=\left(\left(g_{v}\right)_{v \in X^{n}}, \tau_{g}^{(n)}\right)
$$

with $g_{v} \in\left\{1, a, b_{\sigma^{n}(\omega)}, c_{\sigma^{n}(\omega)}, d_{\sigma^{n}(\omega)}\right\} \forall v \in X^{n}$ and $\tau_{g}^{(n)} \in S_{2}$.

Proof By induction on the length of $g$, in the sense of (19).

In Theorem 1.10 of the Introduction, the claim on intermediate growth is a repetition of part of Proposition 4.2, and the claim on covers is the theorem below.

Theorem 4.5 For $\omega \in \Omega_{+}$, any finitely presented cover of $G_{\omega}$ is large.

Remark 4.6 (1) Let $\omega \in \Omega_{-}$. Any finitely presented cover of the infinitely presented group $\widetilde{G}_{\omega}$ contains non-abelian free groups, by Theorem 1.5. As recorded in Proposition 4.2.iii, the group $G_{\omega}$ is virtually free abelian, and finitely presented. For example, if $\omega$ is the constant sequence $000 \cdots$, then $G_{\omega}$ is the infinite dihedral group. 
(2) If we replace "is large" by "contains non-abelian free subgroups" in Theorem 4.5 , the resulting statement has a short proof. Indeed:

For any $\omega \in \Omega$, any finitely presented cover of $\widetilde{G}_{\omega}$ has non-abelian free subgroups. Indeed, let $\left(\omega_{n}\right)_{n \geq 1}$ be a sequence of eventually constant sequences converging to $\omega$ in $\Omega$. Then $\widetilde{G}_{\omega_{n}}$ is virtually metabelian and infinitely presented for all $n \geq 1$ (Claims (i) and (iii) in Proposition 4.2), and $\left(\widetilde{G}_{\omega_{n}}\right)_{n \geq 1}$ converges to $\widetilde{G}_{\omega}$ (Proposition 4.2.v). Let $E$ be a finitely presented cover of $\widetilde{G}_{\omega}$. Then $E$ is a cover of $G_{\omega_{n}}$ for $n$ large enough (Proposition 3.3). Hence Bieri-Strebel Theorem 1.5 shows that $E$ contains non-abelian free subgroups.

From now on, we assume that

$$
\omega \in \Omega_{+} \text {. }
$$

Our strategy for the proof of Theorem 4.5 is to adapt to the present context the steps of Section 2.

The following definition should be compared with Definition 2.10. Note however that $G_{0}$ has not quite the same meaning here and there.

Definition 4.7 Set again

$$
G_{0}=\left\langle a, b, c, d \mid a^{2}, b^{2}, c^{2}, d^{2}, b c d\right\rangle \simeq C_{2} * V,
$$

as in Example 2.16. Observe that any element of $G_{0}$ can be written as

$$
(*) a * a * \cdots a(*)
$$

with $* \in\{b, c, d\},(*) \in\{1, b, c, d\}$, and $n \geq 0$ occurrences of $a$ (compare with Equation (19)).

For $i \in\{0,1,2\}$, set

$$
\varphi_{i}(a)=(1,1) \tau \text { for all } i \in\{0,1,2\}
$$

and

$$
\begin{aligned}
& \varphi_{0}(b)=(a, b) \quad \varphi_{1}(b)=(a, b) \quad \varphi_{2}(b)=(1, b) \\
& \varphi_{0}(c)=(a, c) \quad \varphi_{1}(c)=(1, c) \quad \varphi_{2}(c)=(a, c) \\
& \varphi_{0}(d)=(1, d) \quad \varphi_{1}(d)=(a, d) \quad \varphi_{2}(d)=(a, d) .
\end{aligned}
$$

It is easy to check that these formulas define homomorphisms

$$
\varphi_{i}: G_{0} \longrightarrow G_{0} 2 S_{2} \quad(i=0,1,2) \text {. }
$$

Set $\varphi_{\omega}^{(1)}=\varphi_{\omega_{1}}$ and define, inductively for $n \geq 2$, homomorphisms

$$
\varphi_{\omega}^{(n)}: G_{0} \stackrel{\varphi_{\omega}^{(n-1)}}{\longrightarrow} G_{0} 2^{n-1} S_{2} \stackrel{\varphi_{\omega_{n}} 21_{2^{n}}}{\longrightarrow} G_{0} 2^{n} S_{2} .
$$


For $n \geq 1$, set

$$
N_{\omega, n}=\operatorname{ker}\left(\varphi_{\omega}^{(n)}\right) \text { and } G_{\omega, n}=G_{0} / N_{\omega, n} .
$$

We have natural homomorphisms

$$
\begin{aligned}
\pi_{\omega}: G_{0} & \longrightarrow G_{\omega}, \\
\widehat{\pi}_{\omega}=\widehat{\pi}_{\omega, 1}: G_{0} 2 S_{2} & \longrightarrow G_{\sigma(\omega)} 2 S_{2}, \\
\widehat{\pi}_{\omega, n}: G_{0} 2^{n} S_{2} & \longrightarrow G_{\sigma^{n}(\omega)} 2^{n} S_{2} .
\end{aligned}
$$

(Compare with (9), (10), and (13), but note that $\widehat{\pi}_{\omega, 1}=\pi_{\omega} 21_{2}$ does not hold here.)

The next lemma is about diagrams analogous to (11) and (14). Its proof uses an argument similar to one in the proof of Proposition 2.7, and will be omitted.

Lemma 4.8 The diagram

$$
\begin{array}{cccc}
G_{0} & \stackrel{\varphi_{\omega}^{(n)}}{\longrightarrow} & G_{0} \imath^{n} S_{2} & \\
\pi_{\omega} & \downarrow & \downarrow & \hat{\pi}_{\omega, n} \\
G & \stackrel{\Phi_{\omega}^{(n)}}{\longrightarrow} & G_{\sigma^{n}(\omega)} \imath^{n} S_{2}
\end{array}
$$

commutes for each $n \geq 1$.

The next lemma is analogous to Step 2 in the proof of Proposition 2.7.

Lemma 4.9 (contraction in $G_{0}$ ) For all $k \in G_{0}$, there exists an integer $n \geq 1$ such that

$$
\varphi_{\omega}^{(n)}(k)=\left(\left(k_{v}\right)_{\left.v \in X^{n}, \tau_{k}^{(n)}\right)}\right.
$$

with $k_{v} \in\{1, a, b, c, d\} \forall v \in X^{n}$ and $\tau_{k}^{(n)} \in S_{2}$.

Proof by induction on the length of $k$, in the sense of (20).

Define now

$$
N_{\omega}=\bigcup_{n \geq 1} N_{\omega, n}
$$

(compare with Definition 2.10). The two following lemmas are appropriate modifications of Lemmas 2.12 and 2.13; we repeat the proof for the first one, and not for the second one.

Lemma 4.10 We have

$$
N_{\omega}=\operatorname{ker}\left(\pi_{\omega}: G_{0} \longrightarrow G_{\omega}\right), \text { namely } G_{\omega} \simeq G_{0} / N_{\omega},
$$


so that

$$
\lim _{n \rightarrow \infty} G_{\omega, n}=G_{\omega}
$$

in the space of marked groups on 4 generators.

Proof Let $g \in N$. Let $n \geq 1$ be such that $g \in \operatorname{ker}\left(\varphi_{\omega}^{(n)}\right)$. Since $\Phi_{\omega}^{(n)} \pi_{\omega}(g)=$ $\widehat{\pi}_{\omega, n} \varphi_{\omega}^{(n)}(g)$, we have $\pi_{\omega}(g)=1$ by Lemma 4.4.i.

Conversely, let $k \in G_{0}$. There exists $n \geq 0$ such that $\left(\varphi_{\omega}^{(n)}(k)\right)_{v} \in\{1, a, b, c, d\}$ for all $v \in X^{n}$, by Lemma 4.9. Assume that $k \in \operatorname{ker}\left(\pi_{\omega}\right)$. Then $\widehat{\pi}_{\omega, n}\left(\varphi_{\omega}^{(n)}(k)\right)=1$. As $\widehat{\pi}_{\omega, n}$ is injective "on generators" (in a sense similar to that of Remark 2.11), we have $\varphi_{\omega}^{(n)}(k)=1$, and therefore $k \in N_{\omega, n} \subset N_{\omega}$.

(Note that the hypothesis " $\omega \in \Omega_{+}$" is necessary for the previous argument. If $\omega_{n}$ were eventually constant, one of $b_{\sigma^{n}(\omega)}, c_{\sigma^{n}(\omega)}, d_{\sigma^{n}(\omega)}$ would be the identity of $G_{\sigma^{n}(\omega)}$ for $n$ large enough).

Lemma 4.11 In the situation of the previous lemma, we have for all $n \geq 1$

$$
\varphi_{\omega}^{(1)}\left(N_{\omega, n}\right) \subset N_{\omega, n-1}^{2} \subset G_{0} \prec S_{2} \text { and }\left(\varphi_{\omega}^{(1)}\right)^{-1}\left(N_{\omega, n-1}^{2}\right) \subset N_{\omega, n} .
$$

It follows that $\varphi_{\omega}^{(1)}: G_{0} \longrightarrow G_{0} 2 S_{2}$ induces a homomorphism

$$
\psi_{\omega}^{(n)}:\left\{\begin{array}{l}
G_{\omega, n} \longrightarrow G_{\omega, n-1} \prec S_{2} \\
g N_{\omega, n} \longmapsto\left(\left(\left(\varphi_{\omega_{n}}(g)\right)_{x} N_{\omega, n-1}\right)_{x \in X}, \tau_{g}^{(1)}\right)
\end{array}\right.
$$

which is injective.

Proposition 4.12 For each $\omega \in \Omega_{+}$and $n \geq 0$, the group $G_{\omega, n}$ is large.

Proof The group $G_{0}=C_{2} * V$ has a free subgroup of finite index, indeed a subgroup isomorphic to $F_{3}$ of index 8 . For $n \geq 1$, because of the previous lemma and as in the proof of Theorem 2.14, there exists a subgroup of index 2 in $G_{\omega, n}$ and a homomorphism from this subgroup onto $G_{\omega, n-1}$. It follows by induction on $n$ that $G_{\omega, n}$ is large.

End of proof of Theorem 4.5 Since $G_{\omega, n}$ is large for $n$ large enough, it follows from Lemma 4.10 and Corollary 3.4 that any cover of $G_{\omega}$ is large.

Definition 4.13 For $\omega \in \Omega$, let $M_{\omega}$ denote the kernel of the defining cover $F_{4} \rightarrow G_{\omega}$; in other terms, $M_{\omega}$ is the inverse image of $N_{\omega}$ by the epimorphism $F_{4} \rightarrow G_{0}$ mapping the four generators of $F_{4}$ onto $a, b, c, d \in G_{0}$. For a subset $\Psi$ of $\Omega$, the $\Psi$-universal group is the group

$$
\mathcal{U}_{\Psi}=F_{4} / \bigcap_{\omega \in \Psi} M_{\omega}
$$

For example, $\mathcal{U}_{\emptyset}=\{1\}$, and $\mathcal{U}_{\{\omega\}}=G_{\omega}$ for all $\omega \in \Omega$. 
The terminology is justified by cases that have appeared in the literature, with $\Psi$ large. For example, let $\Lambda$ denote the subset of $\Omega_{0}$ of sequences that are concatenations of blocks $012,120,201$. Then $\mathcal{U}_{\Lambda}$ has uncountably many quotients (a consequence of Proposition 4.2.iv); it has intermediate growth, and therefore is amenable (established in [75, Theorem 9.7]).

Suppose that $\Psi$ contains some $\omega \in \Omega_{+}$. Then any cover of $\mathcal{U}_{\Psi}$ is a cover of $G_{\omega}$. Theorem 4.5 implies:

Corollary 4.14 For any $\Psi \subset \Omega$ such that $\Psi \cap \Omega_{+} \neq \emptyset$, the $\Psi$-universal group $\mathcal{U}_{\Psi}$ is infinitely presented, and any finitely presented cover of it is large.

In particular, this corollary solves the first part of Problem 9.5 in [73], by showing that $\mathcal{U}_{\Omega}$ is infinitely presented.

\section{The group of intermediate growth $\mathfrak{G}$}

Let $\mathfrak{G}$ be the self-similar group of degree 2 of Example 2.16. On the one hand, $\mathfrak{G}$ is a group of the family studied in the previous section: $\mathfrak{G}=G_{\overline{012}}$; thus Theorem 4.5 "contains" Theorem 1.6. On the other hand, in this particular case, we can describe much more precisely a sequence of finitely presented covers converging to $\mathfrak{G}$, and this is the subject of the present section. Note however that, even if the cover $\mathfrak{G}_{-1}$ below is the same as $G_{0}$ in Example 2.16, the sequence $\left(\mathfrak{G}_{n}\right)_{n \geq 0}$ is not the particular case for $\mathfrak{G}$ of the sequence $\left(G_{n}\right)_{n \geq 1}$ (even shifted) of Section 2 .

Immediately after its discovery it was observed that $\mathfrak{G}$ is infinitely presented. Then, Lysenok found a presentation that we recall below.

Set

$$
\mathfrak{G}_{-1}=\left\langle a, b, c, d \mid a^{2}=b^{2}=c^{2}=d^{2}=b c d=1\right\rangle \simeq C_{2} * V
$$

and denote by $S$ the system of four involutions $\{a, b, c, d\}$ generating $\mathfrak{G}_{-1}$. Elements in $\mathfrak{G}_{-1}$ are in natural bijection with "reduced words" of the form

$$
t_{0} a t_{1} a \cdots a t_{k-1} a t_{k}
$$

with $k \geq 0, t_{1}, \ldots, t_{k-1} \in\{b, c, d\}$, and $t_{0}, t_{k} \in\{\emptyset, b, c, d\}$. Throughout the remainder of this section, we use the same symbol to denote an element of $\mathfrak{G}_{-1}$ and its image in any quotient of $\mathfrak{G}_{-1}$, in particular in $\mathfrak{G}$; thus, $S=\{a, b, c, d\}$ denotes a set of generators in $\mathfrak{G}_{-1}$ and in any quotient of $\mathfrak{G}_{-1}$.

The substitution $\sigma$ defined by

$$
\sigma(a)=a c a, \quad \sigma(b)=d, \quad \sigma(c)=b, \quad \sigma(d)=c
$$

extends to reduced words, for example $\sigma(a b a c)=a c a d a c a b$, and the resulting map

$$
\sigma: \mathfrak{G}_{-1} \longrightarrow \mathfrak{G}_{-1}
$$


is a group endomorphism. Define

$$
\begin{aligned}
u_{0}=(a d)^{4} & u_{n}=\sigma^{n}\left(u_{0}\right) \quad \forall n \geq 0 \\
v_{0}=(\text { adacac })^{4} & v_{n}=\sigma^{n}\left(v_{0}\right) \quad \forall n \geq 0 .
\end{aligned}
$$

Theorem $5.1[108,109]$ The group $\mathfrak{G}$ has a presentation

$$
\left\langle a, b, c, d \mid a^{2}=b^{2}=c^{2}=d^{2}=b c d=1, u_{n}=v_{n}=1 \quad \forall n \geq 0\right\rangle .
$$

Note. It is moreover known that this presentation is minimal [71]. Lysenok's presentation if the prototype of what is now called an L-presentation [6].

Definition 5.2 For $n \geq 0$, define a pair $\left(\mathfrak{G}_{n}, S\right) \in \mathcal{M}_{4}$ by

$$
\left.\begin{array}{l}
\mathfrak{G}_{n}=\langle a, b, c, d| \begin{array}{l}
a^{2}=b^{2}=c^{2}=d^{2}=b c d=1 \\
u_{0}=\cdots=u_{n}=v_{0}=\cdots=v_{n-1}=1
\end{array}
\end{array}\right\}
$$

Observe that $\lim _{n \rightarrow \infty}\left(\mathfrak{G}_{n}, S\right)=(\mathfrak{G}, S)$ in $\mathcal{M}_{4}$, and that there are natural surjections $\mathfrak{G}_{-1} \rightarrow \mathfrak{G}_{n} \rightarrow \mathfrak{G}$ for all $n \geq 0$.

Theorem 5.3 For each $n \geq 0$, the group $\mathfrak{G}_{n}$ has a normal subgroup $H_{n}$ of index $2^{2^{n+1}+2}$ which is isomorphic to the direct product of $2^{n}$ free groups of rank 3 .

Remark 5.4 (i) A weaker result was first established in [78]: For each $n \geq 0, \mathfrak{G}_{n}$ contains a subgroup of finite index isomorphic to the direct product of $2^{n}$ copies of finitely generated non-abelian free groups. This by itself implies that any finitely presented cover of $\mathfrak{G}$ contains non-abelian free subgroups.

(ii) The result of [78] was improved in [11]: For each $n \geq 0$, the group $\mathfrak{G}_{n}$ has a normal subgroup $H_{n}$ of index $2^{\alpha_{n}}$, where $\alpha_{n} \leq\left(11 \cdot 4^{n}+1\right) / 3$, and $H_{n}$ is a subgroup of index $2^{\beta_{n}}$ in a finite direct product of $2^{n}$ non-abelian free groups of rank 3 , where $\beta_{n} \leq\left(11 \cdot 4^{n}-8\right) / 3-2^{n}$.

(iii) Our proof of Theorem 5.3 is split in several lemmas, until 5.10.

If $x, \ldots, y$ are elements of a group $H$, we denote by $\langle x, \ldots, y\rangle_{H}$ the subgroup of $H$ they generate, and by $\langle\langle x, \ldots, y\rangle\rangle_{H}$ the normal subgroup of $H$ they generate. Define first

$$
\begin{aligned}
B_{0} & =\langle\langle b\rangle\rangle_{\mathfrak{G}_{0}}, \\
\Xi_{0} & =\langle b, c, d, a b a, a c a, a d a\rangle_{\mathfrak{G}_{0}}, \\
D_{0} & =\langle a, d\rangle_{\mathfrak{G}_{0}}, \\
D_{0}^{\text {diag }} & =\langle(a, d),(d, a)\rangle_{\mathfrak{G}_{0} .} .
\end{aligned}
$$


It is easy to check that $D_{0}^{\text {diag }} \cap\left(B_{0} \times B_{0}\right)=\{1\}$, and that $D_{0}^{\text {diag }}$ normalizes $B_{0} \times B_{0}$. The assignment

$$
\begin{array}{lll}
b \mapsto(a, c) & a b a \mapsto(c, a) \\
c \mapsto(a, d) & \text { aba } \mapsto(d, a) \\
d \mapsto(1, b) & & \text { aba } \mapsto(b, 1)
\end{array}
$$

extends to a group homomorphism $\psi_{0}: \Xi_{0} \longrightarrow \mathfrak{G}_{0} \times \mathfrak{G}_{0}$ [78, Proposition 1]. For each $n \geq 0$, define now

$$
\begin{aligned}
N_{n} & =\left\langle\left\langle u_{0}, \ldots, u_{n}, v_{0}, \ldots, v_{n-1}\right\rangle\right\rangle_{\mathfrak{G}_{0}} ; \text { observe that } N_{n} \subset \Xi_{0} ; \\
\mathfrak{G}_{n} & =\mathfrak{G}_{0} / N_{n} \text { and } \pi_{n}: \mathfrak{G}_{0} \rightarrow \mathfrak{G}_{n} \text { the canonical projection; } \\
B_{n} & =\langle\langle b\rangle\rangle_{\mathfrak{G}_{n}}=\pi_{n}\left(B_{0}\right) ; \\
\Xi_{n} & =\langle b, c, d, a b a, a c a, a d a\rangle_{\mathfrak{G}_{n}}=\pi_{n}\left(\Xi_{0}\right) ; \\
D_{n}^{\text {diag }} & =\langle(a, d),(d, a)\rangle_{\mathfrak{G}_{n} \times \mathfrak{G}_{n}} ; \\
\sigma_{n} & : \mathfrak{G}_{n-1} \longrightarrow \mathfrak{G}_{n}, g N_{n-1} \longmapsto \sigma(g) N_{n} \quad \text { (for } n \geq 1 \text { only). }
\end{aligned}
$$

For the definition of the homomorphism $\sigma_{n}$, note that $\sigma\left(N_{n-1}\right) \subset N_{n}$.

Lemma 5.5 ([78], Lemma 3) Let $B_{0}$ denote the normal subgroup of $\mathfrak{G}_{0}$ generated by b. Then:

(i) $B_{0}$ is of index 8 in $\mathfrak{G}_{0}$;

(ii) $B_{0}$ is generated by the four elements

$\xi_{1}:=b, \xi_{2}:=a b a, \xi_{3}:=$ dabad, $\xi_{4}:=$ adabada;

(iii) $B_{0}$ has the presentation $\left\langle\xi_{1}, \xi_{2}, \xi_{3}, \xi_{4} \mid \xi_{1}^{2}=\xi_{2}^{2}=\xi_{3}^{2}=\xi_{4}^{2}=1\right\rangle$;

(iv) $B_{0}$ contains $N_{n}$ for all $n \geq 1$.

Lemma 5.6 ([78], mostly Proposition 10)

(i) The kernel and the image of the homomorphism $\psi_{0}$ are given by

$$
\begin{aligned}
\operatorname{ker}\left(\psi_{0}\right) & =\left\langle\left\langle u_{1}, v_{0}\right\rangle\right\rangle_{\Xi_{0}}, \\
\operatorname{Im}\left(\psi_{0}\right) & =\left(B_{0} \times B_{0}\right) \rtimes D_{n}^{\text {diag }} \text { of index } 8 \text { in } \mathfrak{G}_{0} \times \mathfrak{G}_{0} .
\end{aligned}
$$

(ii) For $n \geq 1$, the homomorphism $\psi_{0}$ induces an isomorphism

$$
\psi_{n}: \Xi_{n} \stackrel{\simeq}{\longrightarrow}\left(B_{n-1} \times B_{n-1}\right) \rtimes D_{n-1}^{\text {diag }}<8 \mathfrak{G}_{n-1} \times \mathfrak{G}_{n-1}
$$

where $<_{8}$ indicates that the left-hand side is a subgroup of index 8 in the righthand side.

Set $K_{0}=\left\langle\left\langle(a b)^{2}\right\rangle\right\rangle_{\mathfrak{G}_{0}}$; observe that $K_{0} \subset B_{0}$. 
Lemma 5.7 (i) The subgroup $K_{0}$ is of index 2 in $B_{0}$. It is generated by

$$
t=(a b)^{2} \quad v=(b a d a)^{2} \quad w=(a b a d)^{2}
$$

Moreover $K_{0}$ contains $N_{n}$ for $n \geq 1$.

(ii) The group $K_{0}$ is a free group of rank 3.

Proof (i) This follows from [93, p. 230]. Since $B_{0}$ contains $N_{n}$ and each $u_{n}, v_{n}$ is a fourth power, necessarily $N_{n}$ is contained in $K_{0}$.

For (ii), see [11, Proposition 4], where the proof uses Kurosh's theorem. Alternatively one can use the Reidemeister-Schreier method to find a presentation for $K_{0}$ and see that it is indeed free of rank 3.

Lemma 5.8 If $g$ is an element of $B_{n-1}$ then

$$
\psi_{n}\left(\sigma_{n}(g)\right)=(1, g) \text { and } \psi_{n}\left(a \sigma_{n}(g) a\right)=(g, 1) \text {. }
$$

Proof For the generators of $B_{n-1}$ that are images of those of Lemma 5.5 for $B_{0}$, we have

$$
\begin{aligned}
\psi_{n}\left(\sigma_{n}(b)\right) & =\psi_{n}(d)=(1, b) \\
\psi_{n}\left(\sigma_{n}(a b a)\right) & =\psi_{n}(\text { acadaca })=\left(d^{2}, a b a\right)=(1, a b a) \\
\psi_{n}\left(\sigma_{n}(\text { dabad })\right) & =\psi_{n}(\text { cacadacac })=\left(a^{2} a, \text { dabad }\right)=(1, \text { dabad }), \\
\psi_{n}\left(\sigma_{n}(\text { adabada })\right) & =\psi_{n}(\text { acacacadacacaca })=(1, \text { adabada })
\end{aligned}
$$

and this shows the first equality. The second follows because, if $\psi_{n}(h)=\left(h_{0}, h_{1}\right)$, then $\psi_{n}(a h a)=\left(h_{1}, h_{0}\right)$.

Let $K_{n}=K_{0} / N_{n}$. It is a normal subgroup of $\mathfrak{G}_{n}$ contained in $B_{n}$.

\section{Lemma 5.9 Let $n \geq 1$.}

(i) We have $\sigma_{n}\left(K_{n-1}\right) \subset K_{n} \subset B_{n}$.

(ii) If $H_{n-1}$ is a subgroup of $K_{n-1}$, then $\psi_{n}^{-1}\left(H_{n-1} \times H_{n-1}\right) \subset K_{n}$.

Proof (i) Let $t, v, w$ be now the canonical images in $K_{n}$ of the elements of $K_{0}$ denoted by the same symbols in Lemma 5.7. On the one hand, we have $\psi_{n}\left(\sigma_{n}(t)\right)=(1, t)$ by Lemma 5.8. On the other hand, we have

$$
\psi_{n}(w)=\psi_{n}(a b a) \psi_{n}(d) \psi_{n}(a b a) \psi_{n}(d)=(c c, a b a b)=(1, t)
$$

by the definitions of $\psi_{n}$ and $w$. Hence $\sigma_{n}(t)=w \in K_{n}$ by Lemma 5.6.ii.

Let $g_{1} \in \mathfrak{G}_{n-1}$. From the definition of $\psi_{n}$, we see that the composition $\Xi_{n} \longrightarrow$ $\mathfrak{G}_{n-1}$ of $\psi_{n}$ with a projection onto one of the factors is onto. Hence there exists $g \in \Xi_{n}$ and $g_{0} \in \mathfrak{G}_{n-1}$ such that $\psi_{n}(g)=\left(g_{0}, g_{1}\right)$. We have as above ${ }^{10} \psi_{n}\left(\sigma_{n}\left(t^{g_{1}}\right)\right)=\left(1, t^{g_{1}}\right)$ and

$\overline{10}$ Remember that $t^{h}=h^{-1} t h$. 


$$
\psi_{n}\left(w^{g}\right)=\psi_{n}(w)^{\psi_{n}(g)}=(1, t)^{\psi_{n}(g)}=\left(1, t^{g_{1}}\right),
$$

and therefore $\sigma_{n}\left(t^{g_{1}}\right)=w^{g}$. Since $K_{n}$ is a normal subgroup of $\mathfrak{G}_{n}$ containing $w$, we have $\sigma_{n}\left(t^{g_{1}}\right) \in K_{n}$ for all $g_{1} \in \mathfrak{G}_{n-1}$. The inclusion $\sigma_{n}\left(K_{n-1}\right) \subset K_{n}$ follows, because $K_{n-1}$ is generated by $t$ as a normal subgroup of $\mathfrak{G}_{n-1}$.

(ii) Let $\left(h_{0}, h_{1}\right) \in H_{n-1} \times H_{n-1}$. We have

$$
\psi_{n}^{-1}\left(h_{0}, h_{1}\right)=a \sigma_{n}\left(h_{0}\right) a \sigma_{n}\left(h_{1}\right)
$$

by Lemma 5.8, and the right-hand side is in $K_{n}$ by (i).

Set $H_{0}=K_{0}$. For $n \geq 1$, define inductively

$$
H_{n}=\psi_{n}^{-1}\left(H_{n-1} \times H_{n-1}\right)
$$

The definition makes sense by Lemma 5.9.ii. The following lemma finishes the proof of Theorem 5.3.

Lemma 5.10 Let $n \geq 0$, and the notation be as above.

(i) $H_{n}$ is a normal subgroup of $\mathfrak{G}_{n}$ contained in $K_{n}$.

(ii) The group $H_{n}$ is a direct product of $2^{n}$ free groups of rank 3.

(iii) Its index is given by $\left[\mathfrak{G}_{n}: H_{n}\right]=2^{\left(2^{n+1}+2\right)}$.

Proof For $n=0$, the three claims follow from Lemmas 5.5 and 5.7. We suppose now that $n \geq 1$ and that the lemma holds for $n-1$.

(i) The group $H_{n}$ is clearly normal in $\Xi_{n}$, by Lemma 5.6.ii. To show that $H_{n}$ is normal in $\mathfrak{G}_{n}$, it suffices to check that $a H_{n} a \subset H_{n}$, because $\mathfrak{G}_{n}$ is generated by $\Xi_{n}$ (of index 2 in $\left.\mathfrak{G}_{n}\right)$ and $a$. Let $h \in H_{n}$. Let $h_{0}, h_{1} \in H_{n-1}$ be defined by $\psi_{n}(h)=\left(h_{0}, h_{1}\right)$. Then $\psi_{n}(a h a)=\left(h_{1}, h_{0}\right) \in H_{n-1} \times H_{n-1}$, and therefore $a h a \in H_{n}$.

(ii) This is a straightforward consequence of the isomorphism $H_{n} \simeq H_{n-1} \times H_{n-1}$, see again Lemma 5.6.

(iii) By the induction hypothesis, we have

$$
\begin{aligned}
{\left[\left(B_{n-1} \times B_{n-1}\right) \rtimes D_{n-1}^{\text {diag }}: H_{n-1} \times H_{n-1}\right] } \\
\quad=\frac{\left[\mathfrak{G}_{n-1} \times \mathfrak{G}_{n-1}: H_{n-1} \times H_{n-1}\right]}{\left[\mathfrak{G}_{n-1} \times \mathfrak{G}_{n-1}:\left(B_{n-1} \times B_{n-1}\right) \rtimes D_{n-1}^{\mathrm{diag}}\right]} \\
\quad=\frac{2^{2^{n}+2} \times 2^{2^{n}+1}}{2^{3}}=2^{2^{n+1}+1} .
\end{aligned}
$$


Thus the commutative diagram

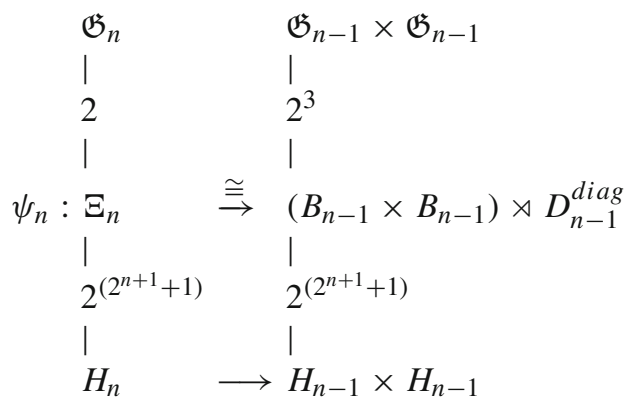

shows that $H_{n}$ has index $2^{\left(2^{n+1}+2\right)}$ in $\mathfrak{G}_{n}$.

The proof of Theorem 5.3 is now complete.

Recall that a group $G$ is called of type $\mathrm{FP}_{n}$ if the trivial $\mathbf{Z}[G]$-module $\mathbf{Z}$ has a projective resolution, namely if there exists an exact sequence

$$
\cdots \longrightarrow P_{j+1} \longrightarrow P_{j} \longrightarrow \cdots \longrightarrow P_{1} \longrightarrow P_{0} \longrightarrow \mathbf{Z}
$$

where the $P_{j}$ 's are projective $\mathbf{Z}[G]$-modules for all $j \geq 0$, and finitely generated projective $\mathbf{Z}[G]$-modules for all $j \leq n$. It is known that

(i) a group is of type $\mathrm{FP}_{1}$ if and only if it is finitely generated;

(ii) finitely presented groups are of type $\mathrm{FP}_{2}$;

(iii) Condition $\mathrm{FP}_{2}$ is strictly weaker than finite presentability;

(iv) a group is of type $\mathrm{FP}_{2}$ if and only if it is the quotient of some finitely presented group by a perfect normal subgroup.

For (i) and (ii), see for example the notes in which "type $\mathrm{FP}_{n}$ " was first defined [28]; see [27] for (iii) and [38, Section VII.5, Exercise 3] for (iv). The following question is natural:

Does $\mathfrak{G}$ have an amenable cover of type $\mathrm{FP}_{2}$ ?

The answer is due to Yves de Cornulier (unpublished). We reproduce it here, with our thanks to him.

Proposition 5.11 (de Cornulier) Any cover of type $\mathrm{FP}_{2}$ of the group $\mathfrak{G}$ is large.

Proof Let $E \rightarrow \mathfrak{G}$ be a cover, with $E$ of type $\mathrm{FP}_{2}$. By (iv) above, there exists a finitely presented group $F$ and a perfect normal subgroup $P$ of $F$ such that $F / P$ is isomorphic to $E$. For $n$ large enough and $\mathfrak{G}_{n}$ as in Theorem 5.3, there exists by Proposition 3.3 a normal subgroup $K_{n}$ of $F$ such that $F / K_{n}$ is isomorphic to $\mathfrak{G}_{n}$. Observe that the group

$$
F / P K_{n} \simeq\left(F / K_{n}\right) /\left(P / P \cap K_{n}\right) \simeq \mathfrak{G}_{n} /\left(P / P \cap K_{n}\right)
$$

is a quotient of $\mathfrak{G}_{n}$ by a perfect normal subgroup. 
Since $\mathfrak{G}_{n}$ has a subgroup of index a power of 2 which is a direct product of free groups (Theorem 5.3, or [11]), $\mathfrak{G}_{n}$ is residually soluble. It follows that the only perfect subgroup of $\mathfrak{G}_{n}$ is $\{e\}$, so that $F / P K_{n} \simeq \mathfrak{G}_{n}$. Hence $F / P K_{n}$ is large, and so is its cover $F / P \simeq E$.

\section{Appendix A: On soluble groups, metabelian groups, and finite presentations}

The existence of groups that are finitely generated and infinitely presented was established by B. H. Neumann in 1937. More precisely, he constructed uncountably many 2-generator groups [120, Theorem 14]; at most countably many of them are finitely presented. Later it was checked that none of them is finitely presented (see the last proof of Appendix C, as well as [26, Theorem C]).

Infinitely generated soluble groups are equally abundant, as we recall below after having fixed some notation.

The groups of the derived series of a group $G$ are defined inductively by $D^{0} G=G$ and $D^{\ell+1} G=\left[D^{\ell} G, D^{\ell} G\right]$. The free soluble group of rank $k$ and solubility class $\ell$ is the quotient $\operatorname{FSol}(k, \ell)=F_{k} / D^{\ell} F_{k}$, where $F_{k}$ stands for the free group of rank $k$. Any $k$-generated soluble group of solubility class at most $\ell$ is a quotient of $\operatorname{FSol}(k, \ell)$. A group $G$ is metabelian if $D^{2} G=1$, namely if it is a cover of an abelian group with abelian kernel. The group $\operatorname{FSol}(k, 2)$ is the free metabelian group of rank $k$.

Philip Hall established the existence of uncountably many finitely generated soluble groups. His result is much more precise [90, Theorem 6]: given any countable abelian group $A \neq 1$, there exist uncountably many groups $G$ such that

$$
d(G)=2, \quad Z(G) \simeq A, \quad\left[G, D^{2} G\right]=1 .
$$

Here $d(G)$ stands for the minimal number of generators of $G$, and $Z(G)$ for its centre. The condition $\left[G, D^{2} G\right]=1$ can be translated in words: $G$ is a centre-by-metabelian group. It is moreover known that there are uncountably many finitely generated soluble groups which are not quasi-isometric to each other [55, Corollary 1.8].

On the contrary, there are only countably many finitely generated metabelian groups (this is repeated as Proposition A. 3 below), and more generally ${ }^{11}$ abelian-bypolycyclic groups ([90, Corollary 2 to Theorem 3], see also [107, Corollary 4.2.5]). Before comparing soluble groups in general with metabelian groups in particular, we collect some well-known facts in the following lemma.

Recall that a group $G$ satisfies Max-n, the maximal condition for normal subgroups, if any increasing sequence of normal subgroups of $G$ is ultimately stationary, or equivalently if any normal subgroup of $G$ is finitely generated as normal subgroup.

Lemma A.1 Let $G$ be a finitely generated group, $N$ a normal subgroup, and $Z$ a central subgroup.

(i) If $G / N$ is finitely presented, there exists a finite subset $S \subset N$ such that $N$ is the smallest normal subgroup of $G$ containing $S$.

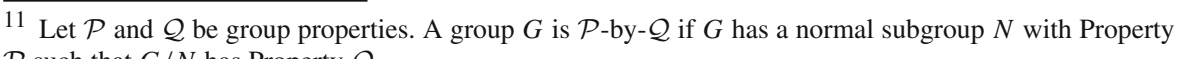
$\mathcal{P}$ such that $G / N$ has Property $\mathcal{Q}$. 
(ii) If $G / Z$ is finitely presented, then $Z$ is finitely generated.

(iii) If $G$ has uncountably many normal subgroups, then $G$ has uncountably many pairwise non-isomorphic quotients.

(iv) Suppose that $G$ is finitely presented and satisfies Max- $n$. Then $G / N$ is finitely presented.

Proof Claim (i) is [132, Lemma 14.1.3]. It is a simple consequence of the following fundamental observation of B.H. Neumann: let $S, S^{\prime}$ be two finite generating sets of a group $G$; assume that $G$ has a finite presentation $\langle S \mid R\rangle$ involving $S$ and a finite set $R$ of relators; then there exists a finite set $R^{\prime}$ of relators in the letters of $S^{\prime}$ such that $\left\langle S^{\prime} \mid R^{\prime}\right\rangle$ is also a finite presentation of $G$ [121, Lemma 8].

Claim (ii) is the special case of (i) for a central subgroup.

For Claim (iii), consider an uncountable family $\left(N_{\alpha}\right)_{\alpha \in A}$ of distinct normal subgroups of $G$. Fix $\alpha \in A$. Let $B$ be a subset of $A$ such that, for each $\beta \in B$, there exists an isomorphism $\phi_{\beta}: G / N_{\beta} \longrightarrow G / N_{\alpha}$. It suffices to show that $B$ is countable.

For $\beta \in B$, let $\pi_{\beta}$ denote the composition of the canonical projection $G \longrightarrow G / N_{\beta}$ with $\phi_{\beta}$. Since $G$ is finitely generated and $G / N_{\alpha}$ countable, there are only countably many homomorphisms from $G$ to $G / N_{\alpha}$. As $N_{\beta}=\operatorname{ker}\left(\pi_{\beta}\right)$, the set $B$ is countable.

For Claim (iv), consider a finite presentation of $G$, namely a free group $F$ on a finite set $S$ and a normal subgroup $M$ of $F$ generated as normal subgroup by a finite subset $R$ of $F$, such that $G=F / M$. Since $G$ satisfies Max-n, there exists a finite subset $R^{\prime}$ of $F$ of which the image in $G$ generates $N$ as a normal subgroup. Then $\left\langle S \mid R \cup R^{\prime}\right\rangle$ is a finite presentation of $G / N$.

Note that Claim (ii) is a special case of [90, Lemma 2]. Our argument for Claim (iii) can be found in [90, p. 433], and that for Claim (iv) is "a well-known principle" cited in [90, p. 420].

Finitely generated metabelian groups are "well-behaved" in many ways:

Proposition A.2 (Hall, Baumslag, Remeslennikov) Let $G$ be a finitely generated metabelian group.

(i) G satisfies Max-n. In particular, the centre of $G$ is finitely generated.

(ii) If $G$ is finitely presented, so is any quotient of $G$.

(iii) $G$ is residually finite.

(iv) G has a soluble word problem.

(v) $G$ can be embedded into a finitely presented metabelian group.

(vi) $G$ is recursively presented.

References Claim (i) is [90, Theorem 3], Claim (ii) follows by Lemma A.1.iv, and Claim (iii) is [91, Theorem 1].

For the particular case of free metabelian groups, Claim (iii) follows from linearity: it is known that $\mathrm{FSol}(k, 2)$ is a subgroup of $\mathrm{GL}_{2}(\mathbf{C})$.

This is a form of the "Magnus embedding theorem" ; see [110], and also [151, Theorem 2.11]. (On the contrary, $\operatorname{FSol}(k, \ell)$ is not linear when $\ell \geq 3$; see Remark A.6.)

Claim (iv) can be found in [20]. It is also a consequence of (a particular case of) a result of Wehrfritz: any finitely generated metabelian group is quasi-linear, namely 
is a subgroup of a group of the form $\prod_{i=1}^{r} \mathrm{GL}_{n}\left(F_{i}\right)$, where $F_{1}, \ldots, F_{r}$ are fields [152]. More generally, several algorithmic problems are known to be soluble in finitely generated metabelian groups [25].

Claim (v) was proved by Baumslag [19] and Remeslennikov [131], independently. See also [107, Proposition 11.3.2].

Claim (vi) is [20, Corollary A1]; it also follows from Claim (v). Note that Claim (vi) is contained in Claim (iv), but we add it for comparison with Proposition A.5.

Proposition A.3 (P. Hall) There are countably many finitely generated metabelian groups.

Remark $A .4$ (a) Let $G$ be a finitely generated metabelian group $G$; let $k \geq 0$ be such that $G$ can be generated by $k$ elements, so that $G$ is a quotient of the free metabelian group FSol $(k, 2)$. Though it need not be finitely presented (examples are shown below), the group $G$ is finitely presented as a metabelian group, because $\operatorname{FSol}(k, 2)$ satisfies Max-n; in other terms, we can write

$$
G=\operatorname{FSol}(k, 2) /\left\langle\left\langle r_{1}, \ldots, r_{n}\right\rangle\right\rangle,
$$

where the notation $\langle\langle\cdots\rangle\rangle$ indicates a normal subgroup generated as such by elements $r_{1}, \ldots, r_{n}$ in $\operatorname{FSol}(k, 2)$. Proposition A.3 follows.

Note that Proposition A.3 is also a straightforward consequence of Claim (vi) in the previous proposition.

(b) Some of the claims in Proposition A.2 can be improved. For example, (i) holds for finitely generated abelian-by-polycyclic groups, and (iii) holds for finitely generated abelian-by-nilpotent groups (Hall). Moreover (iii) holds for abelian-by-polycyclic groups, as shown by Roseblade and Jategaonkar in 1973 and 1974 (see [134], or Chapter 7 and in particular Theorem 7.2.1 in [107]).

(c) Until the early 70's, there were rather few known examples of finitely presented metabelian groups. The 3-generator 3-relator group

$$
H=\left\langle a, s, t \mid a^{t}=a a^{s},[s, t]=1=\left[a, a^{s}\right]\right\rangle
$$

appeared independently in papers by Baumslag [18] and Remeslennikov [131]; see also [145, Theorem A]. It is metabelian, its derived group is free abelian of infinite rank, and it contains the wreath product $\mathbf{Z} \geq \mathbf{Z}$ as a subgroup [20, Pages 72-73]. It was quite a surprise at this time [18, first lines] to find a finitely presented group containing a normal abelian subgroup of infinite rank. More recently, the quotient group $H /\left(a^{2}=1\right)$ was the main character in [77].

For groups of higher solubility degrees, the picture is substantially different, even under the stronger hypothesis of finite presentability. Each of the claims of the next proposition is meant to be compared with the corresponding claim of Proposition A.2.

Proposition A.5 Let $G$ be a finitely presented soluble group.

(i) G need not satisfy Max- $n$. Indeed, the centre of $G$ need not be finitely generated. 
(ii) G may have uncountably many quotients, and in particular infinitely presented quotients.

(ii') Any metabelian quotient of $G$ is finitely presented.

(iii) $G$ need not be residually finite.

(iv) G need not have a soluble word problem.

Let $G$ be now a finitely generated soluble group.

(v) $G$ need not be recursively presented.

(vi) $G$ need not embed into any finitely presented group.

On the proof Let $p$ be a prime. For $n \geq 2$, consider the group $A_{n}$ of $n$-by- $n$ triangular matrices of the form

$$
\left(\begin{array}{ccccc}
1 & * & \cdots & * & * \\
0 & * & \cdots & * & * \\
\vdots & \vdots & \ddots & \vdots & \vdots \\
0 & 0 & \cdots & * & * \\
0 & 0 & \cdots & 0 & 1
\end{array}\right)
$$

with upper-triangular entries $a_{i, j}, 1 \leq i<j \leq n$, in $\mathbf{Z}\left[\frac{1}{p}\right]$, and diagonal entries $a_{2,2}, \ldots, a_{n-1, n-1}$ in $p^{\mathbf{Z}}$. This group is soluble. Its center $Z\left(A_{n}\right)$ is isomorphic to $\mathbf{Z}\left[\frac{1}{p}\right]$, that is to $A_{2}$, and therefore is not finitely generated; it follows that $A_{n}$ does not satisfy Max-n. It is easy to check that $A_{n}$ is finitely generated when $n \geq 3$.

For $n \geq 4$, the group $A_{n}$ is finitely presented ([1] for $n=4$ and [2] for $n \geq 4$ ). This justifies Claim (i). The existence of a finitely presented soluble group without Max-n solves a problem of P. Hall; Remeslennikov had an earlier claim for this [130] which was apparently unjustified [148].

Note that $A_{3}$ is infinitely presented. This was most likely known to P. Hall, and can be found in [2]. But it is also a consequence of Bieri-Strebel Theorem 1.5; indeed, since $Z\left(A_{3}\right)$ is not finitely generated, $A_{3} / Z\left(A_{3}\right)$ is infinitely presented (Lemma A.1), and the soluble group $A_{3}$ cannot be a finitely presented cover of the metabelian group $A_{3} / Z\left(A_{3}\right)$.

Since $Z\left(A_{n}\right) \simeq \mathbf{Z}\left[\frac{1}{p}\right]$ is not finitely generated, the quotient $A_{n} / Z\left(A_{n}\right)$, with $n \geq 3$, is finitely generated non-finitely presented, by Lemma A.1.ii. When $n \geq 4$, this justifies the second part of Claim (ii).

Claim (ii') follows from Theorem 1.5.

For $n \geq 3$, the quotient of $A_{n}$ by the central subgroup

$$
\left\{\begin{array}{l|l}
\left(z_{i, j}\right)_{1 \leq i, j \leq n} \in A_{n} \mid \begin{array}{l}
z_{i, j}=\delta_{i, j} \text { for } \quad(i, j) \neq(1, n) \\
z_{1, n} \in \mathbf{Z}
\end{array}
\end{array}\right\} \simeq \mathbf{Z}
$$

(where $\left(\delta_{i, j}\right)_{1 \leq i, j \leq n}$ denotes the unit matrix) is finitely generated non-Hopfian ${ }^{12}$ (the argument of [92] for $n=3$ carries over to all $n \geq 3$ ), and therefore non-residually finite. When $n \geq 4$, this justify Claim (iii).

12 A group $G$ is non-Hopfian if there exists a surjective endomorphism of $G$ onto itself that is not injective. A finitely generated residually finite group is Hopfian [111]. 
Still for $n \geq 4$, it is known that the quotient $A_{n} / Z\left(A_{n}\right)$ does satisfy Max-n and does not have any minimal presentation [35, Lemma 3.2 and Corollary 3.6]. The last statement means that any presentation of $A_{n} / Z\left(A_{n}\right)$ contains redundant relators; in particular, the finitely related group $A_{n}$ has a quotient that is not finitely related. The group $A_{n}$ itself has only countably many quotients; see [109, Theorem 1] and [35, Corollary 3.4].

Concerning Claim (iv), finitely presented soluble groups with unsoluble word problems have been constructed by Kharlampovich in [102] and by Baumslag, Gildenhuys and Strebel in [24]. Groups in [24] have centers that are not finitely generated, and therefore have infinitely presented quotients (see again Claim (ii)).

Earlier, Meskin had constructed a finitely generated recursively presented residually finite soluble group with unsoluble word problem [116].

For any prime $p$, there exists a finitely presented soluble group $\mathrm{G}$ with centre $\left(C_{p}\right)^{(\infty)}$, an infinite direct sum of cyclic groups of order $p$ (see [103], as well as [104, Lemma 4.14]). Hence $G$ has uncountably many quotients, by Lemma A.1. iii. This justifies the first part of Claim (ii).

Claim (v) follows from the existence of uncountably many finitely generated soluble groups, because there are only countably many recursively presented groups.

(Digression: Let $G$ be a finitely generated soluble group. Assume that $G$ is of finite Prüfer rank, namely that there exists an integer $d \geq 1$ such that any finitely generated subgroup of $G$ can be generated by $d$ elements. Then $G$ is recursively presented if and only if $G$ has a soluble word problem. For this, and for examples of $G$ with and without soluble word problem, see [41].)

Claim (vi) follows from Claim (v) because a finitely generated subgroup of a finitely presented group is recursively presented. (This is straightforward; see the first page of [95], where Higman establishes the famous non-trivial converse; alternatively, see [117, Lemma 2.1].)

Remark A.6 As noted parenthetically just after Proposition A.2, free solvable groups $\operatorname{FSol}(k, \ell)$ are not linear when $k \geq 2$ and $\ell \geq 3$ [141,142]. Here is a proof.

For $k \geq 3$, here is first a short reduction to a more standard result. Let $\mathfrak{C}$ denote the class of (nilpotent-by-abelian)-by-finite groups; observe that quotients of groups in $\mathfrak{C}$ are in $\mathfrak{C}$. The Lie-Kolchin-Mal'cev theorem (see for example [132, Section 15.1]) establishes that soluble linear groups are in $\mathfrak{C}$. If $\operatorname{FSol}(k, \ell)$ were linear, hence in $\mathfrak{C}$, so would be any quotient, in particular the iterated wreath product $\left(\mathbf{Z}_{\imath} \mathbf{Z}\right)<\mathbf{Z}$; but this is not $[132,15.1 .5]$.

The following argument, shown to us by Ralph Strebel, holds for any $k \geq 2$. Denote by $x_{1}, \ldots, x_{k}$ a set of free generators of $\operatorname{FSol}(k, \ell)$. Let $H$ be a subgroup of finite index in $\operatorname{FSol}(k, \ell)$; there exists an integer $m \geq 1$ such that $x_{1}^{m}, x_{2}^{m} \in H$; let $U$ denote the subgroup of $\operatorname{FSol}(k, \ell)$ generated by $x_{1}^{m}$ and $x_{2}^{m}$, so that $U \subset H \subset \subset_{\text {finite index }}$ $\operatorname{FSol}(k, \ell)$. The group $U$ is isomorphic to $\operatorname{FSol}(k, \ell)$, by a result due independently to Gilbert Baumslag [17, Theorem 2] and Shmel'kin [138, Theorem 5.2].

If $\operatorname{FSol}(k, \ell)$ was linear, it would have a nilpotent-by-abelian subgroup of finite index, say $H$. By the lines above, FSol $(2, \ell)$ would be nilpotent-by-abelian. Hence any 2 -generated soluble group of solubility class at least 3 would be nilpotent-byabelian. But this is not true. 
Indeed, consider the symmetric group $S_{4}$ on four letters. It is generated by two elements, a transposition and a 4-cycle. It is soluble of class 3 , with $D^{1} S_{4}=A_{4}$ (the alternating group on four letters), $D^{2} S_{4}=V$ (the Klein Vierergruppe), and $D^{3} S_{4}=\{1\}$. It is not nilpotent-by-abelian, namely $A_{4}$ is not nilpotent: $\left[A_{4}, V\right]=V$.

The argument of Smirnov is different. It relies on the bi-orderability of the groups $\operatorname{FSol}(k, \ell)$.

\section{Appendix B: On wreath products and lamplighter groups}

Permutational wreath products have been defined in the beginning of Section 2. The standard wreath product $G 2_{H} H$ refers to the action of $H$ on itself by left multiplications.

Proposition B.1 Consider two groups $G, H$, a non-empty $H$-set $X$, and the permutational wreath product $G 2_{X} H$. We assume that $G \neq\{1\}$.

(i) $G{ }_{2 x} H$ is finitely generated if and only if $G, H$ are finitely generated and $H$ has finitely many orbits on $X$.

(ii) $G 2_{X} H$ is finitely presented if and only if $G, H$ are finitely presented, the $H_{x}$ 's $(x \in X)$ are finitely generated, and there are finitely many orbits in $X \times X$ for the diagonal action of $H$ (where $H_{x}=\{h \in H \mid h(x)=x\}$ ).

(iii) In particular, as soon as $H$ is infinite, the standard wreath product $G ?_{H} H$ is not finitely presented.

References Claim (i) is standard, and easy; if necessary, see [52, Proposition 2.1]. For Claim (ii), see [52, Theorem 1.1]. Claim (iii) is the main result of [16].

As a particular case of Proposition B.1, if $G$ is finitely-generated abelian and $G \neq 1$, the group $G \geq \mathbf{Z}$ is metabelian, finitely generated, and infinitely presented. When $G$ is finite abelian and $H \simeq \mathbf{Z}$ infinite cyclic, we will call $G>\mathbf{Z}$ the lamplighter group for $G$. (For this terminology, precise assumptions on $G$ and $H$ vary from one author to the other; some ask that $G=\mathbf{Z} / 2 \mathbf{Z}$.)

Proposition B.2 Consider two finitely presented groups $G, H$, an $H$-set $X$ such that $H$ has finitely many orbits on $X$ and infinitely many orbits on $X \times X$, and the permutational wreath product $G{ }_{2 X} H$. We assume that $G \neq\{1\}$.

(i) $G_{2 x} H$ is finitely generated and is infinitely presented.

(ii) For any finitely presented cover $\pi: E \rightarrow G 2_{x} H$, the group $E$ has non-abelian free subgroups.

Proof Claim (i) is a particular case of Proposition B.1. In Proposition 2.10 of [52], it is shown that the kernel of $\pi$ contains non-abelian free subgroups.

Note that, in the particular case of two abelian groups $G$ and $H$, Claim (ii) is also a consequence of Theorem 1.5.

On other proofs of Proposition B.2 in the case of $W:=\mathbf{Z}_{2 \mathbf{Z}} \mathbf{Z}$. (We have $G=H=\mathbf{Z}$ and $X=\mathbf{Z}$.) We have a presentation

$$
W=\left\langle s, t \mid\left[s^{t^{i}}, s^{t^{j}}\right] \forall i, j \in \mathbf{Z}\right\rangle
$$


indeed, any element in the right-hand side can be written as

$$
\begin{aligned}
& s^{m_{1}} t^{n_{1}} s^{m_{2}} t^{n_{2}} s^{m_{3}} t^{n_{3}} \cdots s^{m_{\ell}} t^{n_{\ell}} \\
& \quad=s^{m_{1}}\left(s^{t^{-n_{1}}}\right)^{m_{2}}\left(s^{t^{-n_{1}-n_{2}}}\right)^{m_{3}} \cdots\left(s^{t^{-n_{1}-\cdots-n_{\ell-1}}}\right)^{m_{\ell}} t^{n_{1}+\cdots+n_{\ell}}
\end{aligned}
$$

for some $m_{1}, n_{1}, \ldots, m_{\ell}, n_{\ell} \in \mathbf{Z}$, and therefore as

$$
\left(s^{t^{j_{1}}}\right)^{i_{1}}\left(s^{t^{j_{2}}}\right)^{i_{2}} \cdots\left(s^{t^{j_{k}}}\right)^{i_{k}} t^{N}
$$

for appropriate $i_{1}, j_{1}, \ldots, i_{k}, j_{k}, N \in \mathbf{Z}$ with $t_{1}<t_{2}<\cdots<t_{k}$. It follows that the natural homomorphism

$$
\left\langle s, t \mid\left[s^{t^{i}}, s^{t^{j}}\right] \forall i, j \in \mathbf{Z}\right\rangle \longrightarrow W
$$

is an isomorphism.

Since $t^{i}\left[s, s^{t^{k}}\right] t^{-i}=\left[s^{t^{i}}, s^{t^{i+k}}\right]$, we have a second presentation

$$
W=\left\langle s, t \mid\left[s, s^{t^{i}}\right] \forall i \in \mathbf{N}\right\rangle
$$

For a positive integer $n$, define

$$
W_{n}=\left\langle s, t \mid\left[s, s^{t^{i}}\right], i=0, \ldots, n\right\rangle .
$$

Note that $\lim _{n \rightarrow \infty} W_{n}=W$ in $\mathcal{M}_{2}$. We have a third presentation

$$
W_{n}=\left\langle s_{0}, \ldots, s_{n}, t \mid \begin{array}{l}
{\left[s_{i}, s_{j}\right], \quad 0 \leq i, j \leq n,} \\
s_{k}^{t}=s_{k+1}, \quad 0 \leq k \leq n-1
\end{array}\right\rangle .
$$

Indeed, it can be checked that the assignments

$$
\begin{aligned}
& \varphi_{1}: s \longmapsto s_{0}, t \longmapsto t \\
& \varphi_{2}: s_{i} \longmapsto s^{t^{i}}, t \longmapsto t \quad(0 \leq i \leq n)
\end{aligned}
$$

define, between the groups of the two previous presentations, isomorphisms that are inverse to each other.

Let $H_{n}$ be the free abelian subgroup of $W_{n}$ generated by $s_{0}, \ldots, s_{n}$. Denote by $K_{n}$ the subgroup of $H_{n}$ generated by $s_{0}, \ldots, s_{n-1}$, and by $L_{n}$ that generated by $s_{1}, \ldots, s_{n}$; observe that $K_{n} \simeq L_{n} \simeq \mathbf{Z}^{n}$. Let $\psi_{n}: K_{n} \longrightarrow L_{n}$ be the isomorphism defined by $\psi\left(s_{i-1}\right)=s_{i}$ for $i=1, \ldots, n$. Then $W_{n}$ is clearly the HNN-extension corresponding to the data $\left(H_{n}, \psi_{n}: K_{n} \stackrel{\simeq}{\rightarrow} L_{n}\right)$. By Britton's lemma, $W_{n}$ contains non-abelian free subgroups.

It follows from Corollary 3.4 that any finitely presented cover of $W$ contains nonabelian free groups. 
Let us finally allude to another argument showing that $W$ is infinitely presented. Let $H$ denote the subgroup of $\mathrm{GL}_{3}(\mathbf{C})$ generated by the three matrices

$$
\left(\begin{array}{lll}
1 & 0 & 0 \\
0 & t & 0 \\
0 & 0 & 1
\end{array}\right),\left(\begin{array}{lll}
1 & 1 & 0 \\
0 & 1 & 0 \\
0 & 0 & 1
\end{array}\right) \text {, and }\left(\begin{array}{ccc}
1 & 0 & 0 \\
0 & 1 & 1 \\
0 & 0 & 1
\end{array}\right)
$$

where $t$ is some transcendental number. The centre $Z(H)$ of $H$ is free abelian of infinite rank, and the quotient $H / Z(H)$ is isomorphic to $M:=\mathbf{Z}\left[t, t^{-1}\right]^{2} \rtimes Z$, where $\rtimes$ refers to the action of the generator $1 \in \mathbf{Z}$ by $\left(\begin{array}{ll}t & 0 \\ 0 & t^{-1}\end{array}\right)$. There is an isomorphism of $M$ onto a subgroup of index 2 in $Z\left[t, t^{-1}\right] \rtimes_{t} \boldsymbol{Z} \simeq Z_{\imath} \boldsymbol{Z}$, given by $(P(t), Q(t), n) \mapsto$ $\left(P\left(t^{2}\right)+t Q\left(t^{-2}\right), 2 n\right)$. It follows from Lemma A.1.ii that $\mathbf{Z} \imath \mathbf{Z}$ is not finitely presented. We are grateful to Adrien Le Boudec for correcting an earlier version of our argument at this point.

The isomorphism of $H / Z(H)$ with a subgroup of index 2 in $\mathbf{Z} \imath \mathbf{Z}$ is essentially due to P. Hall. See [90, Theorem 7] and [42, Lemma 3.1].

Concerning Proposition B.2, let us add one more remark about the particular case $W=(\mathbf{Z} / h \mathbf{Z})$ ₹ $\mathbf{Z}$, with $h \geq 2$ : it is known that any finitely presented cover of $W$ is large [21, Section IV.3, Theorem 7].

Recall that the free soluble group $\operatorname{FSol}(k, \ell)=F_{k} / D^{\ell} F_{k}$ of rank $k$ and solubility class $\ell$ has been defined in Appendix A.

Corollary B.3 For $k, \ell \geq 2$, the group $\operatorname{FSol}(k, \ell)$ is infinitely presented, and any finitely presented cover of it contains non-abelian free subgroups.

Note. (i) That FSol $(k, \ell)$ is infinitely presented is a result due to [139]. See also [52, Proposition 2.10 and Corollary 2.14].

(ii) Corollary B.3 and our proof carry over to free polynilpotent groups

$$
\operatorname{FPolynilp}\left(k, \ell_{1}, \ldots, \ell_{n}\right):=F_{k} / C^{\ell_{k}}\left(C^{\ell_{k-1}}\left(\ldots C^{\ell_{1}}\left(F_{k}\right) \cdots\right)\right)
$$

for any $k \geq 2, n \geq 2$, and $\ell_{1}, \ldots, \ell_{n} \geq 2$ (where $C^{\ell} G$ denotes the $\ell$ th group of the lower central series of a group $G$, defined by $C^{1} G=G$, and $C^{\ell+1} G=\left[G, C^{\ell} G\right]$ ). These groups are the subject of [138].

Proof Since $\mathbf{Z}>\mathbf{Z}$ is a two-generator metabelian group, we have an epimorphism FSol $(2,2) \rightarrow \mathbf{Z}$ ? $\mathbf{Z}$. Indeed, we have a sequence of natural epimorphisms

$$
\operatorname{FSol}(k, \ell) \rightarrow \operatorname{FSol}(k, 2) \rightarrow \operatorname{FSol}(2,2) \rightarrow \mathbf{Z} \text { 乙 Z }
$$

Hence any finitely presented cover of $\operatorname{FSol}(k, \ell)$ is also one of $\mathbf{Z} \imath \mathbf{Z}$. If $\operatorname{FSol}(k, \ell)$ was finitely presented, it would contain non-abelian free subgroup by Proposition B.2, but this cannot be in a soluble group.

In the situation of Proposition B.2, suppose moreover that $H$ is infinite residually finite, $G$ has at least one non-trivial finite quotient, and consider the standard wreath 
product $(X=H)$. The following strengthening of Claim (ii) is shown in [54, Theorem 1.5]: any finitely presented cover of $G ? H$ is large. In particular:

any finitely presented cover of $\mathbf{Z} \imath \mathbf{Z}$ is large.

By the proof of Corollary B.3, it follows that,

for $k, \ell \geq 2$, any finitely presented cover of $\operatorname{FSol}(k, \ell)$ is large.

The following notion provides interesting examples of metabelian groups, as we will illustrate with Baumslag-Solitar groups.

Definition B.4 The metabelianization of a group $G$ is the metabelian quotient group $G / D^{2} G$.

Definition B.5 ([22]) For $\ell, m \in \mathbf{Z} \backslash\{0\}$, the Baumslag-Solitar group is defined by the two-generators one-relator presentation

$$
\mathrm{BS}(\ell, m)=\left\langle s, t \mid t^{-1} s^{\ell} t=s^{m}\right\rangle .
$$

We collect three well-known properties of these groups as follows.

Proposition B.6 Let $\ell, m \in \mathbf{Z} \backslash\{0\}$ and $\mathrm{BS}(\ell, m)$ be as above.

(i) $B S(\ell, m)$ is abelian if and only if $B S(\ell, m)$ is nilpotent, if and only if $\ell=m= \pm 1$.

(ii) $B S(\ell, m)$ is metabelian if and only if $B S(\ell, m)$ is soluble, if and only if $B S(\ell, m)$ does not contain non-abelian free subgroups, if and only if $|\ell|=1$ or $|m|=1$.

(iii) If $\ell, m$ satisfy $\ell, m \geq 2$ and are coprime, then $B S(\ell, m)$ is non-Hopfian.

(iv) For $\ell, m$ as in (iii), the group $B S(\ell, m)$ contains non-abelian free groups, but is not large.

On the proof It is easy to check that the four groups $\mathrm{BS}(\ell, m), \mathrm{BS}(m, \ell), \mathrm{BS}(-\ell,-m)$, $\mathrm{BS}(-m,-\ell)$ are isomorphic. For simplicity, let us assume from now on that $\ell$ and $m$ are positive. (For the general case, with all details, we refer to [143]).

It is an exercise to check that $\mathrm{BS}(1, m) \simeq \mathbf{Z}\left[\frac{1}{m}\right] \rtimes_{m} \mathbf{Z}$ for any $m \geq 1$. It follows that $\operatorname{BS}(\ell, m)$ is metabelian if $\ell=1$ or $m=1$, and abelian if and only if $\ell=m=1$. If $m \geq 2$, note that $\mathrm{BS}(1, m)$ is not nilpotent, because its subgroup $\mathbf{Z}\left[\frac{1}{m}\right]$ is not finitely generated. If $\ell \geq 2$ and $m \geq 2$, the subgroup generated by $s^{-1} t s$ and $t$ is free of rank 2, by Britton's Lemma.

Claim (iii) is the main reason for the celebrity of these groups. It is straightforward to check that the assignments $\varphi(s)=s^{\ell}$ and $\varphi(t)=t$ define an endomorphism $\varphi$ of $\mathrm{BS}(\ell, m)$. The image of $\varphi$ contains $t$ and $s^{\ell}$, hence $t^{-1} s^{\ell} t^{-1}=s^{m}$, and therefore $s$; hence $\varphi$ is onto. On the one hand, $\varphi\left(\left[t^{-1} s t, s\right]\right)=\left[t^{-1} s^{\ell} t, s^{\ell}\right]=\left[s^{m}, s^{\ell}\right]=1$; on the other hand, $\left[t^{-1} s t, s\right]=t^{-1} s t s t^{-1} s^{-1} t s^{-1} \neq 1$, where the last inequality holds by Britton's Lemma; hence $\varphi$ is not one-to-one.

More generally, we know necessary and sufficient conditions on $\ell, m$ for $\mathrm{BS}(\ell, m)$ to be non-Hopfian; see [22,50,51].

The first part of Claim (iv) is standard (it also follows from Theorem 1.5, see B.8.ii below). For the second part, see Example 3.2 and Theorem 6 in [58]. 
Definition B.7 For two coprime positive integers $\ell, m$, not both 1 , let

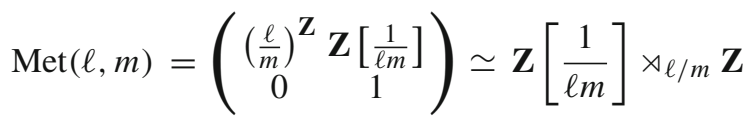

be the group of triangular matrices generated by

$$
\left(\begin{array}{ll}
1 & 1 \\
0 & 1
\end{array}\right) \text { and }\left(\begin{array}{ll}
\frac{\ell}{m} & 0 \\
0 & 1
\end{array}\right)
$$

Let

$$
\mu_{\ell, m}: \operatorname{BS}(\ell, m) \rightarrow \operatorname{Met}(\ell, m)
$$

denote the epimorphism defined by

$$
\mu_{\ell, m}(s)=\left(\begin{array}{ll}
1 & 1 \\
0 & 1
\end{array}\right) \text { and } \mu_{\ell, m}(t)=\left(\begin{array}{cc}
\frac{\ell}{m} & 0 \\
0 & 1
\end{array}\right) .
$$

The following proposition collects facts on $\operatorname{BS}(\ell, m)$ and $\operatorname{Met}(\ell, m)$. Claims (iii) to $(\mathrm{v})$ constitute a digression from our theme.

Proposition B.8 Let the notation be as just above, and $\ell, m$ be two coprime positive integers, not both 1 . For (ii) to (v), assume furthermore that $\min \{l, m\} \geq 2$.

(i) $\mu_{\ell, m}$ is an isomorphism if and only if $\ell=1$ or $m=1$.

(ii) $\operatorname{Met}(\ell, m)$ is infinitely presented.

(iii) The multiplicator group $\mathrm{H}_{2}(\operatorname{Met}(\ell, m), \mathbf{Z})$ is trivial.

(iv) $\operatorname{Met}(\ell, m)$ is of cohomological dimension 3.

(v) For $x \in \mathbf{C}$ transcendental, the matrices $\left(\begin{array}{ll}1 & 1 \\ 0 & 1\end{array}\right)$ and $\left(\begin{array}{ll}x & 0 \\ 0 & 1\end{array}\right)$ generate a group isomorphic to $\mathbf{Z} ? \mathbf{Z}$.

On proofs Claim (i) has already been given as an exercise, in the proof of Proposition B.6. Claim (ii) is a consequence of a particular case of the main result of [23], or a consequence of [30, Theorem C]; see also [107, Proposition 11.4.3]. Claim (iv) is [65, Theorem 4]. Claim (v) is [107, Proposition 3.1.4].

For (iii), see [23, No 1.8]. Recall that, if a group $G$ is finitely presented, then its multiplicator group $\mathrm{H}_{2}(G, \mathbf{Z})$ is finitely generated; this is a simple consequence of the so-called Schur-Hopf Formula, for a group $G=F / R$ presented as a quotient of a free group $F$, which reads " $H_{2}(G, \mathbf{Z})=(R \cap[F, F]) /[R, F]$ ". Claim (iii) is one of the standard examples showing that the converse does not hold.

Let $\ell, m$ be coprime positive integers, with $\ell, m \geq 2$. We denote by $p_{\ell, m}: F_{2} \rightarrow$ $\mathrm{BS}(\ell, m)$ the defining cover of the corresponding Baumslag-Solitar group, namely the cover mapping a basis of the free group of rank 2 onto $\{s, t\}$. Let $\varphi: \operatorname{BS}(\ell, m) \rightarrow$ 
$\mathrm{BS}(\ell, m)$ be the usual non-injective surjective endomorphism, as in Proposition B.6. For $n \geq 1$, set

$$
M_{n}=\operatorname{ker}\left(\varphi^{n}\right), N_{n}=\operatorname{ker}\left(\varphi^{n} p_{m, l}\right), G_{n}=\mathrm{BS}(\ell, m) / M_{n}=F_{2} / N_{n}
$$

Observe that the sequence $\left(M_{n}\right)_{n \geq 1}$ is strictly increasing, yet $G_{n}$ is isomorphic to $\operatorname{BS}(\ell, m)$ for each $n \geq 1$.

Proposition B.9 Let $\ell, m$ be coprime integers, with $\ell, m \geq 2$, and $\operatorname{Met}(\ell, m)$ as in Definition B.7. Let $\left(G_{n}\right)_{n \geq 1}$ be as above.

(i) $\operatorname{Met}(\ell, m)$ is isomorphic to $\mathrm{BS}(\ell, m)_{\text {metab. }}$

(ii) With the notation above, we have an isomorphism

$$
\operatorname{Met}(\ell, m) \simeq F_{2} /\left(\bigcup_{n \geq 1} N_{n}\right)
$$

so that $\lim _{n \rightarrow \infty} G_{n}=\operatorname{Met}(\ell, m)$ in $\mathcal{M}_{2}$.

(iii) Any finitely presented cover of $\operatorname{Met}(\ell, m)$ contains non-abelian free subgroups.

Proof Claim (i) is part of [20, Theorem G].

Claim (ii) is [23, see 1.8] or [76, Theorem 3], there for $(\ell, m)=(2,3)$, but the argument carries over to the case stated here. (When working on [76], the authors were not aware of [23].)

Since BS $(\ell, m)$ contains non-abelian free subgroups by Proposition B.6.ii, and since $\lim _{n \rightarrow \infty} G_{n}=\operatorname{Met}(\ell, m)$, Claim (iii) follows by Corollary 3.4.

\section{Appendix C: On Bieri-Neumann-Strebel invariants}

Example C.1 Consider the two metabelian groups

$$
\begin{aligned}
& \operatorname{Met}(1,6)=\operatorname{BS}(1,6)=\mathbf{Z}[1 / 6] \rtimes_{1 / 6} \mathbf{Z} \\
& \operatorname{Met}(2,3)=\operatorname{BS}(2,3)_{\text {metab }}=\mathbf{Z}[1 / 6] \rtimes_{2 / 3} \mathbf{Z} .
\end{aligned}
$$

The first group is finitely presented. By contrast, the second group is infinitely presented (Propositions B.8). This spectacularly different behavior of two superficially similar-looking groups was the initial motivation of Bieri and Strebel (later joined by Neumann and others) for the work that lead to the $\Sigma$-invariants; see [29], as well as [20, Problem 1]. We come back to these two examples in Example C.3.

Let $G$ be a finitely generated group, $S$ a finite generating set, and $\operatorname{Cay}(G, S)$ the corresponding Cayley graph, with vertex set $G$ and edge set $\left\{\{g, h\} \mid g^{-1} h \in S \cup\right.$ $\left.S^{-1}\right\}$. The set of characters, namely of group homomorphisms from $G$ to $\mathbf{R}$, is a real vector space $\operatorname{Hom}(G, \mathbf{R})$ isomorphic to $\mathbf{R}^{n}$, where $n$ is the torsion-free rank of the abelian group $G / D^{1} G$. Let $S(G)$ denote the character sphere of $G$, quotient of the set of non-zero characters by the natural action of the group of positive reals; we have 
$S(G) \approx \mathbf{S}^{n-1}$, and we write $[\chi]$ the class in $S(G)$ of a character $\chi \neq 0$ in $\operatorname{Hom}(G, \mathbf{R})$. (The sign $\approx$ indicates a homeomorphism; $\mathbf{S}^{k}$ denotes the $k$-sphere, and $\mathbf{S}^{-1}=\emptyset$.) For such a $[\chi]$, set

$$
G_{[\chi]}=\{g \in G \mid \chi(g) \geq 0\},
$$

and let $\operatorname{Cay}_{[\chi]}(G, S)$ be the subgraph of $\operatorname{Cay}(G, S)$ spanned by $G_{[\chi]}$. The BieriNeumann-Strebel invariant, or shortly BNS-invariant, of $G$ is defined to be

$$
\Sigma^{1}(G)=\left\{[\chi] \in S(G) \mid \operatorname{Cay}_{[\chi]}(G, S) \text { is connected }\right\} \subset S(G) .
$$

The superscript indicates that $\Sigma^{1}$ is just one out of many "geometric invariants" (see $[33,146])$.

Observe that there is an antipodal map $[\chi] \longmapsto-[\chi]:=[-\chi]$ defined on $S(G)$. Let $G_{0}$ be a finitely generated group and $p: G_{0} \rightarrow G$ an epimorphism; then $p$ induces a map $p^{*}: S(G) \longrightarrow S\left(G_{0}\right)$ which is a homeomorphism onto its image and which intertwines the antipodal maps.

On the one hand, the invariant of [32] involves one more group $A$ on which $D^{1} G$ acts in an appropriate way; here, we particularize to $A=D^{1} G$. On the other hand, the definition above is a reformulation of the original definition, see for example [146].

Theorem C.2 Let $G$ be a finitely generated group, and $\Sigma^{1}(G) \subset S(G)$ as above.

(i) $\Sigma^{1}(G)$ is independent of the choice of $S$.

(ii) $\Sigma^{1}(G)$ is open in $S(G)$.

(iii) $\Sigma^{1}(G)=S(G)$ if and only if the derived group $D^{1} G$ is finitely generated.

(iv) Suppose that $G$ is metabelian. Then $G$ is finitely presented if and only if $\Sigma^{1}(G) \cup$ $\left(-\Sigma^{1}(G)\right)=S(G)$, and $G$ is polycyclic if and only if $\Sigma^{1}(G)=S(G)$.

(v) If $G$ is finitely presented and has no non-abelian free subgroup, then $\Sigma^{1}(G) \cup$ $\left(-\Sigma^{1}(G)\right)=S(G)$.

(vi) If $G_{0}$ is a finitely generated group and $p: G_{0} \rightarrow G$ an epimorphism, then $\Sigma^{1}\left(G_{0}\right) \cap p^{*}(S(G)) \subset p^{*}\left(\Sigma^{1}(G)\right)$. In particular:

$$
\Sigma^{1}(G) \cup\left(-\Sigma^{1}(G)\right) \varsubsetneqq S(G) \Longrightarrow \Sigma^{1}\left(G_{0}\right) \cup\left(-\Sigma^{1}\left(G_{0}\right)\right) \varsubsetneqq S\left(G_{0}\right) .
$$

On the proof For (i) see [146, Theorem A2.3].

For (ii), see [32, Theorem A] and [146, Theorem A3.3].

(iii) If $D^{1} G$ is finitely generated, then $\Sigma^{1}(G)=S(G)$, [146, Proposition A2.6]. For the "iff", see [32, Theorem B1].

For (iv), see [31, Theorem A] and [146, Subsection B3.2c].

For (v), see [32, Theorem C] and [146, Theorem A5.1].

Claim (vi) is rather straightforward from the definitions [146, Proposition A4.5].

Example C.3 (i) $\Sigma^{1}(G)=S(G) \approx \mathbf{S}^{n-1}$ for $G$ a finitely generated abelian group of torsion-free rank $n$.

(ii) $\Sigma^{1}\left(F_{n}\right)=\emptyset \subset S\left(F_{n}\right) \approx \mathbf{S}^{n-1}$ for the non-abelian free group $F_{n}$ of rank $n \geq 2$; see [146, Item A2.1a]. 
(iii) For the soluble Baumslag-Solitar group $\mathrm{BS}(1, n)$, the invariant $\Sigma^{1}(\mathrm{BS}(1, n))$ is one of the two points of the sphere $S(\mathrm{BS}(1, n)) \approx \mathbf{S}^{0}$; the argument of [146, Item A2.1a] for $n=2$ carries over to $n \geq 2$.

(iv) For two coprime integers $\ell, m \geq 2$, we have $\Sigma^{1}(\operatorname{Met}(\ell, m))=\emptyset \subset \mathbf{S}^{0}[146$, Item A3.6].

(v) If $G$ is a semi-direct product $H \rtimes \mathbf{Z}$ of an infinite locally finite group $H$ by an infinite cyclic group, then $\Sigma^{1}(G)=\emptyset \subset \mathbf{S}^{0}$ [146, Lemma B3.1].

(vi) For any $n \geq 1$ and any rational polyhedral subset $P$ of $\mathbf{S}^{n-1}$, there exists a finitely presented group $G$ with $S(G) \approx \mathbf{S}^{n-1}$ and a homeomorphism $p^{*}: \mathbf{S}^{n-1} \longrightarrow$ $S(G)$ such that $\Sigma^{1}(G)=p^{*}(S(G) \backslash P)$.

Some comment is in order for (vi), cited here to show that the invariant $\Sigma^{1}(G)$ can be more complicated than those of Examples (i) to (v). For a finitely generated group $G$, a non-zero character $\chi \in \operatorname{Hom}(G, \mathbf{R})$ is rational if $\chi(G)$ is an infinite cyclic subgroup of $\mathbf{R}$. Denote by $\operatorname{Hom}_{\mathbf{Q}}^{*}(G, \mathbf{R})$ the set of non-zero rational characters on $G$, and by $S_{\mathbf{Q}}(G)$ its image in $S(G)$; then $S_{\mathbf{Q}}(G)$ is a dense subset of $S(G)$ [146, Lemma B3.3].

Consider a positive integer $n$ and the sphere $\mathbf{S}^{n-1}=S\left(\mathbf{Z}^{n}\right)$. A rational hemisphere of $\mathbf{S}^{n-1}$ is the closure of the image of the half-space $\left\{\chi \in \operatorname{Hom}_{\mathbf{Q}}^{*}(G, \mathbf{R}) \mid \chi(z) \geq 0\right\}$, for some $z \in \mathbf{Z}^{n} \backslash\{0\}$. A rational convex polyhedral subset of $\mathbf{S}^{n-1}$ is a finite intersection of rational hemispheres. A rational polyhedral subset of $\mathbf{S}^{n-1}$ is a finite union of rational convex polyhedral subsets.

Given an integer $n \geq 1$ and a rational polyhedral subset $P \subset \mathbf{S}^{n-1}$, there is a finitely presented group $G$ and an epimorphism $p: G \rightarrow \mathbf{Z}^{n}$ such that $p^{*}: S\left(\mathbf{Z}^{n}\right) \longrightarrow S(G)$ is a homeomorphism, and $\Sigma^{1}(G)=p^{*}\left(S\left(\mathbf{Z}^{n}\right) \backslash P\right)$. See [32, Corollary 7.6] and [34, Chapter IV, Section 1.1].

Corollary C.4 Let $G$ be a finitely generated group and $E$ a finitely presented cover of $G$. If $\Sigma^{1}(G) \cup\left(-\Sigma^{1}(G)\right) \varsubsetneqq S(G)$, then $E$ contains non-abelian free subgroups. This holds in particular:

when $G$ is metabelian and infinitely presented,

when $G=H \rtimes \mathbf{Z}$ with $H$ infinite locally finite, as in Example C.3.v.

Proof This is straightforward from Claims (iii) to (v) of Theorem C.2 and from Example C.3.v.

In Corollary C.4, the claim concerning metabelian groups is precisely Theorem 1.5.

Example C.5 (B.H. Neumann) Let $\mathcal{V}=\left\{2 \leq v_{1}<v_{2}<v_{3}<\cdots\right\}$ be an infinite increasing sequence of integers. Set

$$
X_{\mathcal{V}}=\left\{(i, j) \in \mathbf{Z}^{2} \mid i \geq 1,-v_{i} \leq j \leq v_{i}\right\}
$$

For each $i \geq 1$, denote by $X_{\mathcal{V}, i}$ the subset $\left\{(i, j) \in X_{\mathcal{V}} \mid-v_{i} \leq j \leq v_{i}\right\}$, of cardinal $2 v_{i}+1$. Define two permutations $\alpha_{\mathcal{V}}, \beta_{\mathcal{V}}$ of the set $X_{\mathcal{V}}$ as follows: for each $i \geq 1$, they preserve $X_{\mathcal{V}, i}$, and

$\left(\alpha_{\mathcal{V}}\right)$ the restriction of $\alpha_{\mathcal{V}}$ to $X_{\mathcal{V}, i}$ is the $\left(2 v_{i}+1\right)$-cycle $\left(\left(i,-v_{i}\right),\left(i,-v_{i}+1\right), \ldots,\left(i, v_{i}\right)\right)$, 
$(\beta \mathcal{V})$ the restriction of $\beta \mathcal{V}$ to $X_{\mathcal{V}, i}$ is the 3-cycle $((i,-1),(i, 0),(i, 1))$.

The Neumann group corresponding to $\mathcal{V}$ is the group $G_{\mathcal{V}}$ of permutations of $X_{\mathcal{V}}$ generated by $\alpha_{\mathcal{V}}$ and $\beta \mathcal{V}$.

Let $\operatorname{Alt}_{f}(\mathbf{Z})$ denote the group of permutations of finite supports of $\mathbf{Z}$ that are even on their support. Let $\operatorname{Alt}_{f}(\mathbf{Z}) \rtimes_{\text {shift }} \mathbf{Z}$ denote its semi-direct product with $\mathbf{Z}$, where $\mathbf{Z}$ acts on itself by shifts, the generator 1 acting by $\alpha: j \longmapsto j+1$. Observe that $\operatorname{Alt}_{f}(\mathbf{Z}) \rtimes_{\text {shift }} \mathbf{Z}$ is generated by $\alpha$ and the 3 -cycle $\beta=(-1,0,1)$. It is easy to check that the assignment $\alpha \mathcal{V} \longmapsto \alpha, \beta_{\mathcal{V}} \longmapsto \beta$ extends to an epimorphism $\pi_{\mathcal{V}}: G_{\mathcal{V}} \rightarrow \operatorname{Alt}_{f}(\mathbf{Z}) \rtimes_{\text {shift }} \mathbf{Z}$. Neumann has shown that the kernel of $\pi_{\mathcal{V}}$ is the restricted product $\prod_{i=1}^{\prime \infty} \operatorname{Alt}\left(2 v_{i}+1\right)$; see [121], as well as [93, Complement III.35].

This has two straightforward consequences. On the one hand, any minimal finite normal subgroup of $G \mathcal{V}$ is one of the $\operatorname{Alt}\left(2 v_{i}+1\right)$. Thus, for two distinct sequences $\mathcal{V}$ and $\mathcal{V}^{\prime}$, the groups $G_{\mathcal{V}}$ and $G_{\mathcal{V}^{\prime}}$ are not isomorphic. In particular, there are uncountably many pairwise non-isomorphic 2-generator groups; hence these are infinitely presented, except possibly for a countable number of them (but see below). On the other hand, the groups $G_{\mathcal{V}}$ are elementary amenable.

Corollary C.6 Let $\mathcal{V}$ be a sequence of integers as in Example C.5. Any finitely presented cover of $G_{\mathcal{V}}$ contains non-abelian free subgroups.

In particular $G_{\mathcal{V}}$ is not finitely presented.

Proof The claim of Corollary C.4 concerning $H \rtimes \mathbf{Z}$ applies to the group Alt $f(\mathbf{Z}) \rtimes_{\text {shift }}$ $\mathbf{Z}$. Since any cover of $G \mathcal{V}$ is a cover of $\operatorname{Alt}_{f}(\mathbf{Z}) \rtimes_{\text {shift }} \mathbf{Z}$, Corollary C.6 follows from Corollary C.4.

Let us also indicate how the last statement of Corollary C.6 is a straightforward consequence of the first paper [30] on BNS-invariants.

Proof that $G_{\mathcal{V}}$ is not finitely presented. It follows from the definition of $G_{\mathcal{V}}$ that the kernel $N_{\mathcal{V}}$ of the composition $G_{\mathcal{V}} \rightarrow \operatorname{Alt}_{f}(\mathbf{Z}) \rtimes_{\text {shift }} \mathbf{Z} \rightarrow \mathbf{Z}$ is locally finite. Suppose (ab absurdo) that $G_{\mathcal{V}}$ is finitely presented. By [30, Theorem A], we have $G \mathcal{V}=\operatorname{HNN}(H, \varphi: K \stackrel{\simeq}{\rightarrow} L)$ for a finitely generated subgroup $H$ of $N_{\mathcal{V}}$ and an isomorphism $\varphi$ between two subgroups $K, L$ of $H$. Since non-ascending extensions contain non-abelian free subgroups (by Britton's lemma), $G_{\mathcal{V}}$ is an ascending HNNextension; we may assume that $K=H$. Since $N_{\mathcal{V}}$ is locally finite, the finitely generated subgroup $H$ of $N_{\mathcal{V}}$ is finite. It follows that $K=H=L$. Hence $G_{\mathcal{V}}=H \rtimes_{\varphi} \mathbf{Z}$ and the kernel of $G_{\mathcal{V}} \rightarrow \mathbf{Z}$ is finite. This is preposterous, and the proof is complete.

\section{Appendix D: On growth and amenability}

Let $G$ be a group generated by a finite set $S$. For an integer $n \geq 0$, let $B_{S}^{G}(n)$ denote the "ball of radius $n$ around the origin", namely the set of those elements $g \in G$ that can be written as words $g=s_{1} \cdots s_{n}$, with $s_{1}, \ldots, s_{n} \in S \cup S^{-1} \cup\{1\}$. Let $\gamma_{S}^{G}(n)$ denote the cardinality of $B_{S}^{G}(n)$. Then $G$ is said to be

(pol) of polynomial growth if there exist constants $a, d>0$ such that $\gamma_{S}^{G}(n) \leq a n^{d}$ for all $n>0$, 
(exp) of exponential growth if there exist a constant $c>1$ such that $\gamma_{S}^{G}(n) \geq c^{n}$ for all $n \geq 0$,

(int) of intermediate growth in other cases.

It is easy to check that this trichotomy depends only on $G$, not on the finite generating set $S$. For information on the growth of groups, we refer to the books [93] and [113].

A group $G$ is amenable if there exists a left-invariant finitely additive probability measure defined on all subsets of $G$ (there are many other equivalent definitions). Two basic results are important here: (i) amenability of groups is preserved by the four operations of taking subgroups, quotients, directs limits, and extensions with amenable kernels (already in [123]), and (ii) groups of intermediate growth are amenable (this goes back to [3], and is also a straightforward consequence of Følner's Criterion [63]). These results make it natural to define three classes of groups:

$\mathcal{A G}$ is the class of amenable groups, defined in [123].

$\mathcal{E} \mathcal{G}$ is the class of elementary amenable groups, defined in [56]; it is the smallest class of groups containing the easiest examples, that are finite groups and abelian groups, and stable by the four operations listed above.

$\mathcal{S G}$ is the class of subexponentially amenable groups (see below for an historical comment on this definition); it is the smallest class of groups containing $\mathcal{E} \mathcal{G}$ and the next easiest examples, that are the groups of intermediate growth.

We have a partition

$$
\mathcal{A G}=(\mathcal{A G} \backslash \mathcal{S G}) \sqcup(\mathcal{S G} \backslash \mathcal{E G}) \sqcup \mathcal{E G}
$$

Let us mention a few groups in each of these three parts.

The class $\mathcal{E} \mathcal{G}$ contains all virtually soluble groups and all locally finite groups; other examples are cited in Section 1.A.

There are finitely generated groups in $\mathcal{E} \mathcal{G}$ which are not virtually soluble: for example all Neumann groups $G_{\mathcal{V}}$ discussed in Example C.5, or an example in [97]. As already mentioned in Section 1.D, any countable elementary amenable group embeds in a finitely generated elementary amenable group; the same hold for "amenable" instead of "elementary amenable" [128, Corollary 1.3].

Let us describe a family of finitely presented elementary amenable groups that are not virtually soluble.

Example D.1 Consider an integer $n \geq 1$, the set

$$
S_{n}=\left\{(j, k) \in \mathbf{Z}^{2} \mid j \geq 0,1 \leq k \leq n\right\}
$$

of $n$ parallel half-intervals in the square lattice, and the Houghton group $H_{n}$ of all permutations $h$ of $S_{n}$ such that, for each $k \in\{1, \ldots, n\}$, there exists a translation $t_{k} \in \mathbf{Z}$ such that $h(j, k)=\left(j+t_{k}, k\right)$ for all $j$ large enough [98]. Denote by $\operatorname{Sym}_{f}\left(S_{n}\right)$ the group of permutations of $S_{n}$ with finite supports, clearly a normal subgroup of $H_{n}$, and set 


$$
A=\left\{\left(t_{1}, \ldots, t_{n}\right) \in \mathbf{Z}^{n} \mid \sum_{k=1}^{n} t_{k}=0\right\} .
$$

We have a short exact sequence

$$
1 \longrightarrow \operatorname{Sym}_{f}\left(S_{n}\right) \longrightarrow H_{n} \stackrel{\pi}{\longrightarrow} A \longrightarrow 1
$$

where, with the notation above, $\pi(h)=\left(t_{1}, \ldots, t_{n}\right)$. Since $\operatorname{Sym}_{f}\left(S_{n}\right)$ is locally finite and $A$ abelian, $H_{n}$ is elementary amenable.

It is known that the group $H_{n}$ is of type $\mathrm{FP}_{n-1}$ but not $\mathrm{FP}_{n}$ [39, Theorem 5.1]. In particular, for $n \geq 3$, the group $H_{n}$ is finitely presented.

Finitely generated groups in the class $\mathcal{E} \mathcal{G}$ are either of polynomial growth or of exponential growth [48]; this has been sharpened: a finitely generated group in the class $\mathcal{E} \mathcal{G}$ has either polynomial growth or uniform exponential growth [129] (see also [37]). By a famous theorem of Gromov [87], a finitely generated group of polynomial growth is virtually nilpotent, and in particular finitely presented.

The class $\mathcal{S G} \backslash \mathcal{E} \mathcal{G}$ contains the class of finitely generated groups of intermediate growth. Historically, the group $\mathfrak{G}$ of Theorem 1.6 and Example 2.16 was the first group shown to be of intermediate growth [67]. This class also contains finitely presented groups, such as the group with 5 generators and 11 relators of [70], later shown to have another presentation with 2 generators and 4 relators (due to Bartholdi, see [44, Number 12]).

The history of early papers on the class $\mathcal{S G}$ is worth a few lines. This class was first implicitly introduced in a paper on 4-manifold topology, more precisely on 4-manifold surgery and 5-dimensional s-cobordism theorems, [64] (see also [106]), and then explicitly in [70]. Freedman and Teichner introduce a class of groups that they call "good", defined as the groups for which the " $\pi_{1}$-Null Disk Lemma" holds; this lemma establishes the existence of 2-discs bounding some closed curves in 4-manifolds of a certain kind. Good groups include finitely generated groups in the class $\mathcal{S G}$ [64, Theorem 0.1 and Lemma 1.2].

The class $\mathcal{A G} \backslash \mathcal{S G}$ contains the Basilica group $\mathfrak{B}$ of Example 2.17, which was first shown to be not in $\mathcal{S G}$ [80], and later shown to be amenable [10]. The method of Bartholdi and Virag was streamlined and generalized by Kaimanovich in [101], in terms of entropy and the legendary "Münchhausen's trick". This and later papers show the amenability of $\mathfrak{B}$ and of many other non elementary amenable groups (see [5,13], building among other things on [140]). The class $\mathcal{A G} \backslash \mathcal{S G}$ contains also the finitely generated amenable simple groups that appear in [100], and in Problem 1.12.

Non-amenable groups include non-abelian free groups, more generally groups containing non-abelian free subgroups [123].

We conclude this report by Question D.2, due to Tullio Ceccherini-Silberstein. Before this, we recall some background. 
A group $G$ has a paradoxical decomposition if there exist integers $p, q \geq 2$, subsets $X_{1}, \ldots, X_{p}, Y_{1}, \ldots, Y_{q}$ of $G$, and elements $g_{1}, \ldots, g_{p}, h_{1}, \ldots, h_{q}$ in $G$ such that

$$
\begin{aligned}
G & =X_{1} \sqcup \cdots \sqcup X_{p} \sqcup Y_{1} \sqcup \cdots Y_{q} \\
& =g_{1} X_{1} \sqcup \cdots \sqcup g_{p} X_{p}=h_{1} Y_{1} \sqcup \cdots \sqcup h_{q} Y_{q},
\end{aligned}
$$

where $\sqcup$ denotes disjoint union. Tarski has shown that $G$ is non-amenable if and only if $G$ has a paradoxical decomposition [147]; see also [150] and [94]. For $G$ non-amenable, the Tarski number $\mathcal{T}(G)$ of $G$ is the minimum of the sum $p+q$, over all paradoxical decompositions of $G$. When $G$ has non-abelian free subgroups, it is easy to show that $\mathcal{T}(G)=4$; in particular, if $G$ is a non-elementary Gromovhyperbolic group, then $\mathcal{T}(G)=4$. As a student of Tarski in the 1940's, Jonsson has shown that, conversely, $\mathcal{T}(G)=4$ implies that $G$ has non-abelian free subgroups. It is also easy to show that $\mathcal{T}(G) \geq 6$ for a non-amenable torsion group $G$. See [150] or [44, Propositions 20 and 21]. We do not know any example of a non-amenable group $G$ without non-abelian free subgroups for which the exact value of $\mathcal{T}(G)$ has been computed.

Let $\mathcal{M}_{m}^{\text {na }}$ be the subspace of $\mathcal{M}_{m}$ of those pairs $(G, S)$ with $G$ non-amenable. For $m \geq 2$, the Tarski number function

$$
\mathcal{M}_{m}^{\mathrm{na}} \longrightarrow \mathbf{N},(G, S) \longmapsto \mathcal{T}(G)
$$

is not continuous. Indeed, there are sequences of non-elementary Gromov-hyperbolic groups (with Tarski number 4) converging in $\mathcal{M}_{m}$ to non-amenable torsion groups (with Tarski number at least 6). See [46, Théorème 1.3], or one of the following classes of examples.

(1) For integers $m, n \geq 2$, let $\mathrm{B}(m, n)$ denote the free Burnside group of rank $m$ and exponent $n$, that is the quotient of the free group $F_{m}$ by the set of relators $\left(w^{n}=1\right)_{w \in F_{m}}$. For $n$ odd and $n \geq 665$, Adyan has shown that $\mathrm{B}(m, n)$ is nonamenable [4]; moreover, it is known that $6 \leq \mathcal{T}(\mathrm{B}(m, n)) \leq 14$ [44, Theorem 61].

Such groups are limits in $\mathcal{M}_{m}$ of non-elementary Gromov-hyperbolic groups. More precisely, for $n$ odd and large enough, the group $\mathrm{B}(m, n)$ is a limit in $\mathcal{M}_{m}$ of a sequence $(\mathrm{B}(m, n, i))_{i \geq 1}$ of non-elementary Gromov-hyperbolic groups. A similar fact is shown by Ivanov in [99, see Lemma 21.1], in the much more difficult case of $n$ even, with $n \geq 2^{48}$ and $n$ divisible by $2^{9}$; his proof adapts to the case needed here, with important simplifications (compare with [85], in particular Theorems 1.10 and 1.7, and recall that a finitely presented group with a subquadratic Dehn function is hyperbolic). In particular, for $m \geq 2$ and $n$ odd large enough, the free Burnside group $\mathrm{B}(m, n)$, of Tarski number between 6 and 14, is a limit in $\mathcal{M}_{m}$ (indeed in $\mathcal{M}_{m}^{\text {na }}$ ) of groups of Tarski number 4.

(2) Ol'shanskii has worked out several "Tarski monster groups". In particular, for any prime $p$ large enough, he has constructed a 2-generated non-amenable torsion group $\operatorname{TM}(p)$ in which any proper non-trivial subgroup is of order $p$. Moreover TM $(p)$, of Tarski number $\geq 6$, is a limit in $\mathcal{M}_{2}$ of non-elementary Gromov hyperbolic groups, of Tarski number 4 [124, Lemma 10.7a]. 
In several other papers by Ol'shanskii and co-authors, there are several other classes of examples of such Burnside type limits of hyperbolic groups. Let us only quote one paper [127], and the book [125].

Question D.2 For an integer $k \geq 4$ and a finitely generated non-amenable group $G$ with $\mathcal{T}(G)=k$, does there exist a finitely presented cover $E$ of $G$ with $\mathcal{T}(E)=k$ ?

The answer is clearly positive when $\mathcal{T}(G)=4$ (with $E$ free). If we define $\mathcal{T}(G)=$ $\infty$ when $G$ is amenable, the previous question for $k=\infty$ coincides with Question 1.1 , and the answer is negative.

Acknowledgments We are most grateful to Yves de Cornulier and Ralph Strebel, for generous comments on preliminary versions of this paper, as well as to Laurent Bartholdi, Gilbert Baumslag, Marc Burger, Tullio Ceccherini-Silberstein, Anna Erschler, Sergei Ivanov, Olga Kharlampovich, Volodymyr Nekrashevych, Alexander Ol'shanskii, Vitalii Roman'kov, and Mark Sapir, for useful discussions and mail exchanges.

Open Access This article is distributed under the terms of the Creative Commons Attribution License which permits any use, distribution, and reproduction in any medium, provided the original author(s) and the source are credited.

\section{References}

1. Abels, H.: An example of a finitely presented solvable group. In: Homological group theory. Proc. Sympos. Durham, 1977. London Math. Soc. Lecture Note Ser. 36, 205-211 (1979).

2. Abels, H., Brown, K.S.: Finiteness properties of solvable S-arithmetic groups: an example. J. Pure Appl. Algebra 44, 77-83 (1987)

3. Adel'son-Vel'skii, G.M., Sreider, Y.A.: The Banach mean on groups. Usphei Math. Nauk. (N.S.) 12(6), 131-136 (1957)

4. Adyan, S.I.: Random walks on free periodic groups. Math. USSR Izv. 21(3), 425-434 (1983). [Russian: Izv. Adak. Nauk SSSR, Ser. Math. 46(6), 1139-1149 (1982)]

5. Amir, G., Angel, O., Virag, B.: Amenability of linear-activity automaton groups, Preprint, arXiv:0905.2007v2, 21 June 2011. J. Eur. Math. Soc. (2013, to appear)

6. Bartholdi, L.: L-presentations and branch groups. J. Algebra 268, 419-443 (2003)

7. Bartholdi, L.: (Self-)similar groups and the Farrell-Jones conjectures, Preprint, arXiv:1107.5339v2, 28 Jul 2011

8. Bartholdi, L., Grigorchuk, R.: On parabolic subgroups and Hecke algebras of some fractal groups. Serdica Math. J. 28, 47-90 (2002)

9. Bartholdi, L., Grigorchuk, R., Nekrashevych, V.: From fractal groups to fractal sets. In: Fractal in Graz 2001, pp. 25-118. Birkhäuser, Basel (2003)

10. Bartholdi, L., Virág, B.: Amenability via random walks. Duke Math. J. 130, 39-56 (2005)

11. Bartholdi, L., de Cornulier, Y.: Infinite groups with large balls of torsion elements and small entropy. Arch. Math. Basel 87, 104-112 (2006)

12. Bartholdi, L., Pochon, F.: On growth of torsion groups. Groups Geom. Dyn. 3, 525-539 (2009)

13. Bartholdi, L., Kaimanovich, V., Nekrashevych, V.: On amenability of automata groups. Duke Math. J. 154, 575-598 (2010)

14. Bartholdi, L., Erschler, A.: Growth of permutational extensions. Invent. Math. 189, 431-455 (2012)

15. Bartholdi, L., Erschler, A.: Groups of given intermediate word growth. Preprint, arXiv:1110.3650v2, 15 Nov 2011

16. Baumslag, G.: Wreath products and finitely presented groups. Math. Z. 75, 22-28 (1961)

17. Baumslag, G.: Some subgroup theorems for free $v$-groups. Trans. AMS 108, 516-525 (1963)

18. Baumslag, G.: A finitely presented metabelian group with a free abelian derived group of infinite rank. Proc. Am. Math. Soc. 35, 61-62 (1972)

19. Baumslag, G.: Subgroups of finitely presented metabelian groups. J. Australian Math. Soc. 16, 98-110 (1973) 
20. Baumslag, G.: Finitely presented metabelian groups. In: Proc. Conf. Theory of Groups, Canberra, 1973. Springer Lecture Notes Math. 372, 65-74 (1974)

21. Baumslag, G.: Topics in combinatorial group theory. Lectures in Mathematics. Birkhäuser, ETH Zürich (1993)

22. Baumslag, G., Solitar, D.: Some two-generator one-relator non-Hopfian groups. Bull. Am. Math. Soc. 68, 199-201 (1962)

23. Baumslag, G., Strebel, R.: Some finitely generated, infinitely related metabelian groups with trivial multiplicator. J. Algebra 40, 46-62 (1976)

24. Baumslag, G., Gildenhuys, D., Strebel, R.: Algorithmically insoluble problems about finitely presented solvable groups, Lie and associative algebras. I. J. Pure Appl. Algebra 39, 53-94 (1986)

25. Baumslag, G., Cannonito, F.B., Robinson, D.J.S.: The algorithmic theory of finitely generated metabelian groups. Trans. Am. Math. Soc. 344, 629-648 (1994)

26. Baumslag, G., Miller, C.F., III.: Reflections on some groups of B. H. Neumann. J. Group Theory 12, 771-781 (2009)

27. Bestvina, M., Brady, N.: Morse theory and finiteness properties of groups. Invent. Math. 129, 445-470 (1997)

28. Bieri, R.: Homological dimension of discrete groups. Queen Mary College, Mathematical Notes (1976)

29. Bieri, R.: Finitely presented soluble groups. Séminaire d'algèbre Paul Dubreuil, 1977-1978. Springer Lecture Notes Math. 740, 1-8 (1979)

30. Bieri, R., Strebel, R.: Almost finitely presented soluble groups. Comment. Math. Helv. 53, 258-278 (1978)

31. Bieri, R., Strebel, R.: Valuations and finitely presented metabelian groups. Proc. Lond. Math. Soc. 41(3), 439-464 (1980)

32. Bieri, R., Neumann, W., Strebel, R.: A geometric invariant of discrete groups. Invent. Math. 90, 451-477 (1987)

33. Bieri, R., Renz, B.: Valuations on free resolutions and higher geometric invariants of groups. Comment. Math. Helv. 63, 464-497 (1988)

34. Bieri, R., Strebel, R.: Geometric invariants for discrete groups, unpublished book (1992) (see [146])

35. Bieri, R., de Cornulier, Y., Guyot, L., Strebel, R.: Infinite presentability of groups and condensations, Preprint, arXiv:1010.0271v2, 17 May 2012

36. Bourbaki, N.: Intégration, chapitres 7 et 8, Hermann, Paris (1963)

37. Breuillard, E.: On uniform exponential growth for solvable groups. Pure Appl. Math. Q. 3, 949-967 (2007)

38. Brown, K.S.: Cohomology of groups. Graduate Text in Math 87, Springer, Berlin (1982)

39. Brown, K.S.: Finiteness properties of groups. J. Pure Appl. Algebra 44, 45-75 (1987)

40. Bux, K.-U., Pérez, R.: On the growth of iterated monodromy groups. In: Topological and asymptotic aspects of group theory. Contemp. Math. 394, 61-76 (Amer. Math. Soc. 2006)

41. Cannonito, F.B., Robinson, D.J.S.: The word problem for finitely generated soluble groups of finite rank. Bull. Lond. Math. Soc. 16, 43-46 (1984)

42. Caprace, P.-E., de Cornulier, Y.: On embeddings into compactly generated groups, Preprint, 17 September 2012

43. Ceccherini-Silberstein, T., Coornaert, M.: Cellular automata and groups. Springer, Berlin (2010)

44. Ceccherini-Silberstein, T., Grigorchuk, R., de la Harpe, P.: Amenability and paradoxical decompositions for pseudogroups and for discrete metric spaces. Proc. Steklov Inst. Math. 224, 57-97 (1999)

45. Chabauty, C.: Limite d'ensembles et géométrie des nombres. Bull. Soc. Math. France 78, 143-151 (1950)

46. Champetier, C.: L'espace des groupes de type fini. Topology 39, 657-680 (2000)

47. Champetier, C., Guirardel, V.: Limit groups as limits of free groups: compactifying the set of free groups. Isarel J. Math. 146, 1-75 (2005)

48. Chou, C.: Elementary amenable groups. Illinois J. Math. 1, 396-407 (1980)

49. Clapham, C.R.J.: Finitely presented groups with word problems of arbitrary degrees of insolubility. Proc. Lond. Math. Soc. 14, 633-676 (1964)

50. Collins, D.J.: Some one-relator Hopfian groups. Trans. Am. Math. Soc. 235, 363-374 (1978)

51. Collins, D.J., Levin, F.: Automorphisms and Hopficity of certain Baumslag-Solitar groups. Arch. Math. 40, 385-400 (1983) 
52. de Cornulier, Y.: Finitely presented wreath products and double coset decompositions. Geom. Dedicata 122, 89-108 (2006)

53. de Cornulier, Y., Guyot, L., Pitsch, W.: On the isolated points in the space of groups. Groups Geom. Dyn. 1, 47-59 (2007)

54. de Cornulier, Y., Kar, A.: On Property (FA) for wreath products. J. Group Theory 14, 165-174 (2011)

55. de Cornulier, Y., Tessera, R.: Dehn function and asymptotic cones of Abels' group, Preprint, arXiv: 1203.4696v1, 21 Mar 2012

56. Day, M.M.: Amenable semigroups. Illinois J. Math. 1, 509-544 (1957)

57. Dunwoody, M.J., Minasyan, A.: An (FA)-group that is not (FR), Preprint, arXiv:1203.3317v1, 15 Mar 2012

58. Edjvet, M., Pride, S.J.: The concept of "largeness" in group theory II, In: Kim, A.C., Neumann, B.H. (eds.) Groups-Korea 1983. Lecture Notes in Mathematics, vol. 1098, pp. 29-54. Springer, Berlin (1984)

59. Erschler, A.: Non residually finite groups of intermediate growth, commensurability and nongeometricity. J. Algebra 222, 154-172 (2004)

60. Erschler, A.: Automatically presented groups. Groups Geom. Dyn. 1, 47-59 (2007)

61. Fabrykowski, J., Gupta, N.: On groups with sub-exponential growth functions. J. Indian Math. Soc. 49, 249-256 (1985)

62. Fabrykowski, J., Gupta, N.: On groups with sub-exponential growth functions II. J. Indian Math. Soc 56, 217-228 (1991)

63. Følner, E.: On groups with full Banach mean value. Math. Scand. 3, 243-254 (1955)

64. Freedman, M.H., Teichner, P.: 4-manifold topology I: subexponential groups. Invent. Math. 122, 500-529 (1995) (See the Appendix in [106])

65. Gildenhuys, D.: Classification of soluble groups of cohomological dimension two. Math. Z. 166, 21-25 (1979)

66. Grigorchuk, R.: On Burnside's problem on periodic groups. Funktsional. Anal. i Prilozhen 14, 53-54 (1980)

67. Grigorchuk, R.: On the Milnor problem of growth. Dokl. Acad. Nauk SSSR 271, 30-33 (1983)

68. Grigorchuk, R.: Degrees of growth of finitely generated groups and the theory of invariant means, Izv. Akad. Nauk SSSR Ser. Mat. 48(5), 939-985 (1984) [In English Math. USSR Izv. 85, 259-300 (1985)]

69. Grigorchuk, R.: Construction of $p$-groups of intermediate growth that have a continuum of quotient groups. Algebra Logic 23, 265-273 (1984)

70. Grigorchuk, R.: An example of a finitely presented amenable group that does not belong to the class EG. Mat. Sb. 189, 79-100 (1998)

71. Grigorchuk, R.: On the system of defining relations and the Schur multiplier of periodic groups generated by finite automata. In: Campbell, C.M. et al. (eds.) Groups St Andrews 1997 in Bath, vol. 1. London Math. Soc. Lecture Note Ser. 260, 290-317 (1999)

72. Grigorchuk, R.: Just infinite branch groups. In: du Sautoy, M., et al. (eds.) New horizons in pro-pgroups. Birkhäuser, Basel (2000)

73. Grigorchuk, R.: Solved and unsolved problems around one group. In: Bartholdi, L., CeccheriniSilberstein, T., Smirnova-Nagnibeda, T., Żuk, A. (eds.) Infinite groups: geometric, combinatorial and dynamical aspects. Progr. Math, vol. 248, pp. 117-218. Birkhäuser Basel (2005)

74. Grigorchuk, R.: Hanoi Towers group on 3 pegs and its pro-finite closure, joint work with Volodymyr Nekrashevych and Zoran Šunić, Mathematisches Forschungsinstitut Oberwolfach Report 25/2006, pp. 1477-1479 [see also Report 19/2006, pp. 1179-1182]

75. Grigorchuk, R.: Milnor's Problem on the growth of groups and its consequences, Preprint, arXiv:1111.0512v2, 31 Jan 2012

76. Grigorchuk, R., Mamaghani, M.J.: On use of iterates of endomorphisms for constructing groups with specific properties. Mat. Stud. 8, 198-206, 238 (1997)

77. Grigorchuk, R., Linnell, P., Schick, T., Żuk, A.: On a question of Atiyah. C.R. Acad. Sci., Paris, Sér. I, Math. 331(9), 663-668 (2000)

78. Grigorchuk, R., de la Harpe, P.: Limit behaviour of exponential growth rates for finitely generated groups. Essays on geometry and related topics. Mémoires dédiés à André Haefliger, vol. 2. Monogr. Enseign. Math. 38, 351-370 (2001)

79. Grigorchuk, R.: Żuk, A.: The lamplighter group as a group generated by a 2-state automaton, and its spectrum. Geom. Dedicata 87, 209-244 (2001) 
80. Grigorchuk, R.: Żuk, A.: On a torsion-free weakly branch group defined by a three state automaton. Int. J. Algebra Comput. 12, 223-246 (2002)

81. Grigorchuk, R., Żuk, A.: Spectral properties of a torsion-free weakly branch group defined by a three state automaton. In: Computational and statistical group theory (Las Vegas, 2001). Contemp. Math., vol. 298, pp. 57-82. Amer. Math. Soc., New York (2002)

82. Grigorchuk, R., Šunić, Z.: Asymptotic aspects of Schreier graphs and Hanoi Towers groups. C. R. Math. Acad. Sci. Paris 342(8), 545-550 (2006)

83. Grigorchuk, R., Šunić, Z.: Self-similarity and branching in group theory. In: Campbell, C.M. et al. (eds.) Groups St. Andrews 2005, vol. 1. London Math. Soc. Lecture Note Ser. 339, 36-95 (2007)

84. Grigorchuk, R., Savchuk, D., Šunić, Z.: The spectral problem, substitutions and iterated monodromy. In: Probability and Mathematical Physics. CRM Proc. Lecture Notes, vol. 42, pp. 225-248. Amer. Math. Soc., New York (2007)

85. Grigorchuk, R., Ivanov, S.V.: On Dehn functions of infinite presentations of groups. Geom. Funct. Anal. 18, 1841-1874 (2009)

86. Grigorchuk, R., Medynets, K.: Topological full groups are locally embeddable into finite groups, Preprint, arXiv:1105.0719v3, 23 Jan 2012

87. Gromov, M.: Groups of polynomial growth and expanding maps (with an appendix by Jacques Tits). Publ. math. I.H.É.S. 53, 53-78 (1981)

88. Gupta, N., Sidki, S.: On the Burnside problem for periodic groups. Math. Z. 182, 385-388 (1983)

89. Hall, M.: A topology for free groups and related groups. Ann. Math. 52, 127-139 (1950)

90. Hall, P.: Finiteness conditions for soluble groups. Proc. Lond. Math. Soc. 4, 419-436 (1954) [Collected Works, pp. 305-324]

91. Hall, P.: On the finiteness of certain soluble groups. Proc. Lond. Math. Soc. 9, 595-622 (1959) [Collected Works, pp. 515-544]

92. Hall, P.: The Frattini subgroups of finitely generated groups. Proc. Lond. Math. Soc. 11, 327-352 (1961) [Collected Works, pp. 581-608]

93. de la Harpe, P.: Topics in geometric group theory. Chicago University Press, Chicago (2000)

94. de la Harpe, P., Skandalis, G.: Un résultat de Tarski sur les actions moyennables de groupes et les partitions paradoxales. Enseign. Math 32, 121-138 (1986)

95. Higman, G.: Subgroups of finitely presented groups. Proc. R. Soc. Lond. Ser. A 262, 455-475 (1961)

96. Higman, G., Neumann, B.H., Neumann, H.: Embedding theorems for groups. J. Lond. Math. Soc. 24, 247-254 (1949)

97. Hillman, J.A.: Elementary amenable groups and 4-manifolds with Euler characteristic 0. J. Austral. Math. Soc. (Series A) 50, 160-170 (1991)

98. Houghton, C.H.: The first cohomology of a group with permutation module coefficients. Arch. Math 31, 254-258 (1978)

99. Ivanov, S.V.: The free Burnside groups of sufficiently large exponents. Int. J. Algebra Comput. 4(1-2), 1-308 (1994)

100. Juschenko, K., Monod, N.: Cantor systems, piecewise translations and simple amenable groups, Preprint, arXiv:1204.2132v3, 29 Apr 2012

101. Kaimanovich, V.: "Münchhausen trick" and amenability of self-similar groups. Int. J. Algebra Comput. 15, 907-937 (2005)

102. Kharlampovich, O.: A finitely presented solvable group with unsolvable word problem, Izv. Akad. Nauk SSSR Ser. Math. 45, 852-873, 928 (1981). [English translation: Math. USSR Izvestija 19 (1982), 151-169]

103. Kharlampovich, O.: The word problem for groups and Lie algebras, Doctor's Thesis. Russian), Moscow Steklov Mathematical Institute (1990)

104. Kharlampovich, O., Myasnikov, A., Sapir, M.: Algorithmically complex residually finite groups, Preprint, arXiv:1204.6506v4, 4 Oct 2012

105. The Kourovka notebook: unsolved problems in group theory, 16th edn. Russian Academy of Sciences, Siberian Division (2006)

106. Kruskhal, V.S., Quinn, F.: Subexponential groups in 4-manifold topology. Geom. Topol. 4, 407-430 (2000) [With an appendix by Michael H. Freedman and Peter Teichner]

107. Lennox, J.C., Robinson, D.J.S.: The theory of infinite soluble groups. Clarendon Press, Oxford (2004)

108. Lysënok, I.: A set of defining relations for the Grigorchuk group. Math. Zametki 38, 503-516, 636 (1985) [English: Math. Notes Acad. Sc. USSR 38(4), 784-792 (1985)]

109. Lyul'ko, N.A.: Normal subgroups of Abels' groups. Math. Notes 36, 639-641 (1984) 
110. Magnus, W.: On a theorem of Marshall Hall. Ann. Math. 40, 764-768 (1939)

111. Mal'cev, A.I.: On the faithful representation of infinite groups by matrices. Am. Math. Soc. Transl. Ser. 2, 45, 1-18 (1962) [Russian: Rec. Math. [Mat. Sbornik] N.S., 8(50), 405-422 (1940)]

112. Mandelbrot, B.B.: Fractal aspects of the iteration of $z \rightarrow \Lambda z(1-z)$ for complex $\Lambda$ and $z$. Ann. N. Y. Acad. Sci. 357, 249-259 (1980)

113. Mann, A.: How groups grow. Cambridge Univ Press, Cambridge (2011)

114. Matui, H.: Some remarks on topological full groups of Cantor minimal systems. Int. J. Math. 17, 231-251 (2006)

115. Meldrum, J.D.P.: Wreath products of groups and semigroups. Pitman Monographs and Survey in Pure and Applied Mathematics 74, Longman, Harlow, 1995

116. Meskin, S.: A finitely generated residually finite group with an unsolvable word problem. Proc. Am. Math. Soc. 43, 8-10 (1974)

117. Miller, C.F., III.: Decision problems for groups-survey and reflections. In: Algorithms and classification in combinatorial group theory (Berkeley, CA, 1989), pp. 1-59. Springer, Berlin (1991)

118. Nekrashevych, V.: Self-similar groups. Mathematical Surveys and Monographs, vol. 117. American Mathematical Society, Providence (2005)

119. Nekrashevych, V.: Free subgroups in groups acting on rooted trees. Groups Geom. Dyn. 4, 847-862 (2010)

120. Nekrashevych, V.: Iterated monodromy groups. In: Campbell, C.M. et al. (eds.) Groups St Andrews 2009 in Bath, vol. 1. London Math. Soc. Lecture Note Ser. 387, 41-93 (2011)

121. Neumann, B.H.: Some remarks on infinite groups. J. Lond. Math. Soc. 12, 120-127 (1937)

122. Neumann, B.H., Neumann, H.: Embedding theorems for groups. J. Lond. Math. Soc. 34, 465-479 (1959)

123. von Neumann, J.: Zur allgemeinen Theorie des Masses, Fund. Math. 13 (1929), 73-116, 333 [Collected works, Vol. I, pp. 599-643]

124. Ol'shanskii, A.Y.: An infinite group with subgroups of prime orders. Mth. USSR, Izv. 16, 279-289 (1981) [Izv. Akad. Nauk SSSR, Ser. Mat. 44, 309-321 (1980)]

125. Ol'shanskii, A.Y.: Geometry of defining relations in groups. Kluwer, Dordrecht (1991)

126. Ol'shanskii, A.Y., Sapir, M.: Non-amenable finitely presented torsion-by-cyclic groups. Publ. Math. I.H.É.S. 96, 43-169 (2002)

127. Ol'shanskii, A.Y., Osin, D., Sapir, M.: Lacunary hyperbolic groups (with an appendix by Michael Kapovich and Bruce Kleiner). Geom. Topol. 13, 2052-2140 (2009)

128. Ol'shanskii, A.Y., Osin, D.: A quasi-isometric embedding theorem for groups, Preprint, arXiv:1202.6437v2, 2 Mar 2012

129. Osin, D.: Algebraic entropy of elementary amenable groups. Geom. Dedicata 104, 133-151 (2004)

130. Remeslennikov, V.N.: A finitely presented soluble group without maximum condition for normal subgroups. Mat. Zametki 12, 287-293 (1972) [Russian]

131. Remeslennikov, V.N.: On finitely presented soluble groups. In: Proc. Fourth All-Union Symposion on the Theory of Groups (Novosibirsk), pp. 164-169 (1973) [Russian]

132. Robinson, D.J.S.: A course in the theory of groups, 2nd edn. Graduate Texts in Math. vol. 80. Springer, Berlin (1996)

133. Sapir, M., Wise, D.T.: Ascending HNN extensions of residually finite groups can be non-Hopfian and can have very few finite quotients. J. Pure Appl. Algebra 166, 191-202 (2002)

134. Segal, D.: On abelian-by-polycyclic groups. J. Lond. Math. Soc. 11, 445-452 (1975)

135. Serre, J.-P.: Cohomologie des groupes discrets. In: Prospects in Mathematics. Annals of Math. Studies, vol. 70, pp. 77-169. Princeton Univ. Press, Princeton (1971)

136. Serre, J.-P.: Arbres, amalgames, SL2, Astérisque, vol. 46. Soc. Math, France (1977)

137. Shalom, Y.: Rigidity of commensurators and irreducible lattices. Invent. Math. 141, 1-54 (2000)

138. Shmelkin, A.L.: Free polynilpotent groups. Am. Math. Transl. II 55, 270-304 (1966) [Russian: Izv. Akad. Nauk. SSSR 28, 91-122 (1964)]

139. Shmelkin, A.L.: Über auflösbare Produkte von Gruppen. Sib. Mat. Sz. 6, 212-220 (1965)

140. Sidki, S.: Automorphisms of one-rooted trees: growth, circuit structure and acyclicity. J. Math. Sci. New York 100, 1925-1943 (2000)

141. Smirnov, D.M.: Generalized solvable groups and their group rings. Soviet Math. Doklady 5, 465-467 (1964)

142. Smirnov, D.M.: On generalized solvable groups and their group rings. Mat. Sb. (N.S.) 67(109), 366383 (1965) 
143. Souche, E.: Quasi-isométries et quasi-plans dans l'étude des groupes discrets, Ph.D. Thesis, Université de Provence, 17 décembre 2001. Appendice A: groupes de Baumslag-Solitar, pp. 69-89 (2001)

144. Stepin, A.M.: Approximation of groups and group actions, the Cayley topology. In: Ergodic theory of $Z_{d}$-actions: Proceedings of the Warwick symposium, 1993-94, Lect. Note Ser. 228, pp. 475-484. Cambridge University Press, Cambridge (1996)

145. Strebel, R.: Finitely presented soluble groups. In: Group theory, Essays for Philip Hall, pp. $257-314$. Academic Press, New York (1984)

146. Strebel, R.: Notes on the Sigma-invariants, Part 1, Preprint, arXiv:1204.0214v1, 1 Apr 2012

147. Tarski, A.: Algebraische Fassung des Massproblems. Fund. Math. 31, 47-66 (1938) [Collected papers, vol. 1, pp. 599-643.]

148. Thomson, M.W.: Subgroups of finitely presented solvable linear groups. Trans. Am. Math. Soc. 231, 133-142 (1977)

149. Valette, A.: A short proof that free groups are residually p-groups. Expositiones Math. 11, 65-71 (1993)

150. Wagon, S.: The Banach-Tarski paradox. Cambridge University Press, Cambridge (1985)

151. Wehrfritz, B.A.F.: Infinite linear groups, Ergebnisse der Mathematik und ihrer Grenzbebiete, vol. 76. Springer, Berlin (1973)

152. Wehrfritz, B.A.F.: On finitely generated soluble linear groups. Math. Zeit. 170, 155-167 (1980) 